\title{
A GEOMETRIC FRAMEWORK FOR STABILIZATION BY ENERGY SHAPING: SUFFICIENT CONDITIONS FOR EXISTENCE OF SOLUTIONS*
}

\author{
BAHMAN GHARESIFARD ${ }^{\dagger}$, ANDREW D. LEWIS ${ }^{\ddagger}$, AND ABDOL-REZA MANSOURI ${ }^{\S}$
}

\begin{abstract}
We present a geometric formulation for the energy shaping problem. The central objective is the initiation of a more systematic exploration of energy shaping with the aim of determining whether a given system can be stabilized using energy shaping feedback. We investigate the partial differential equations for the kinetic energy shaping problem using the formal theory of partial differential equations. The main contribution is sufficient conditions for integrability of these partial differential equations. We couple these results with the integrability results for potential energy shaping [25]. This gives some new avenues for answering key questions in energy shaping that have not been addressed to this point.
\end{abstract}

1. Introduction. In Brockett's 1977 paper [9] it was observed that there were structural aspects of mechanical systems that made them attractive as a class of control problems. In this paper he mentioned differential geometry as the common mathematical structure between control theory and analytical mechanics. He investigated the Lagrangian and Hamiltonian formulations for mechanical systems and considered the interplay of the mechanical and control theoretic structures.

One interesting control problem is the following: given a mechanical system with an unstable equilibrium at a point $q_{0}$, stabilize the system using feedback. One of the recent developments in the stabilization of equilibria is the energy shaping method. The key idea concerns the construction of a feedback for which the closed-loop system possesses the structure of a mechanical system. A feedback so obtained is called an energy shaping feedback and the procedure by which it is obtained is called energy shaping. In the classical notion of energy shaping, the assumed method consists of two stages: shaping the kinetic energy of the system-so-called kinetic energy shaping - and changing the potential energy of the system - so-called potential energy shaping. If such an energy shaping feedback exists, then for stability one has to ensure that the Hessian of the closed-loop potential energy is positive definite.

The cart-pendulum, as a mechanical system with one degree of underactuation, is one of the systems that has been stabilized using the energy shaping method [14, 26]. The system has the upright equilibria as saddle points and potential energy

\footnotetext{
*Dedicated to Roger Brockett on the occasion of his 70th birthday.

${ }^{\dagger} \mathrm{PhD}$ student, Department of Mathematics and Statistics, Queen's University, URL: http://www.mast.queensu.ca/ gharesifard/

${ }^{\ddagger}$ Associate Professor, Department of Mathematics and Statistics, Queen’s University, URL: http://penelope.mast.queensu.ca/ andrew/

$\S$ Assistant Professor, Department of Mathematics and Statistics, Queen's University, URL: http://www.mast.queensu.ca/ mansouri/
} 
shaping alone is not enough to stabilize the system; therefore kinetic energy shaping is necessary. More complicated mechanical systems with more degrees of freedom like the spherical pendulum have been stabilized using the energy shaping method [8]. It is worth mentioning that the spherical pendulum has been mentioned as an example of a mechanical control system in Brockett's 1977 paper [9].

To the authors' knowledge there has been no linearly controllable example (note that linear controllability is a necessary condition for stabilization by energy shaping) in the literature that could be proved to be not stabilizable by the energy shaping method. For linear systems, linear controllability is also a sufficient condition for existence of a stabilizing feedback [40,29]. Such sharp conditions for nonlinear systems do not exist in the literature. Thus the question of which mechanical systems are stabilizable using energy shaping is still unresolved. Moreover, almost all the existing results on energy shaping are based on a specific parametrization of the assumed solutions to the energy shaping problem. While the parameterizations used are sufficient for the particular problems, it is not clear whether (1) a better controller would result if a richer class of feedbacks were available or (2) there are systems that are not presently amenable to stabilization by energy shaping using existing parameterizations, but which could be stabilized using energy shaping were the complete set of energy shaping feedbacks known.

In this paper we give a geometric framework for kinetic energy shaping that should help to answer some of the key questions about stabilization of mechanical systems using energy shaping. Recently there have been notable attempts to investigate various features of the energy shaping problem. The first classical appearance of the notion of potential energy shaping problem is in [35]. Van der Schaft had a significant geometric contribution to the problem from the Hamiltonian point of view [37]. It turns out that this method has an extension in the Lagrangian setting called the method of controlled Lagrangians; this has been investigated by Bloch, Leonard, Marsden, Chang, $[8,7]$. In recent work, Chang, Woolsey and others have realized that the space of possible kinetic energy feedbacks can be enlarged by considering the addition of appropriate gyroscopic forcing $[13,39]$. In the Hamiltonian framework, the idea of kinetic energy shaping has been related in [15] to the notion of interconnection and modified into the of IDA-PBC method [26]. The equivalence of the Controlled Lagrangian method and the IDA-PBC method has been addressed in $[14,6]$. Both methods result in a set of partial differential equations whose solutions determine the energy shaping feedbacks. In other recent work, the possibility of finding a coordinate change for simplifying the kinetic energy shaping partial differential equations in the IDA-PBC method has been investigated [38]. Lewis in [24] reformulated the kinetic energy shaping problem as the problem of finding an energy preserving connection with its associated closed-loop metric.

A differential geometric approach to the kinetic energy shaping problem - the so- 
called $\lambda$-method

- has been presented in [5]. In this paper the authors propose a system of linear partial differential equations for the kinetic energy shaping problem in terms of a new variable, $\lambda=\mathbb{G}_{\mathrm{cl}}^{\sharp} \mathbb{G}_{\mathrm{ol}}^{b}$, where $\mathbb{G}_{\mathrm{ol}}$ and $\mathbb{G}_{\mathrm{cl}}$ are the open-loop and closed-loop metrics, respectively. The main idea of the $\lambda$-method is that it transforms the set of quasilinear equations for kinetic energy shaping into a set of overdetermined linear partial differential equations [3]. In [4] an equivalent system of linear partial differential equations is given for the assumed procedure of kinetic energy shaping problem. Moreover, the authors investigate the compatibility conditions for the set of $\lambda$-equation in local coordinates. However, the analysis of the compatibility conditions is not complete, and many structural questions remain unanswered, even after one accounts for the results in $[3,4]$. The $\lambda$-method has been modified by adding the possibility of using gyroscopic forces for enlarging the space of solutions [13].

Lewis [24] has introduced an affine differential geometric approach to energy shaping in order to have a better geometric understanding of the problem and to state some of the questions that had not been addressed before. The main idea of the approach involves first understanding the existence of such an energy shaping feedback and then what such a feedback might look like. In recent work, sufficient conditions for the existence of potential energy shaping are derived assuming that kinetic energy shaping has taken place [25]. The results are based on the integrability theory for linear partial differential equations developed by Goldschmidt [18] and Spencer [34]. Although the results offer some insight, they are limited by the fact that kinetic energy shaping has been assumed to precede potential energy shaping.

In the present work, we use the affine differential geometric approach for modeling mechanical systems. We consider the class of simple mechanical control systems. The central objective of this paper is the initiation of a more systematic geometric exploration of energy shaping with the aim of determining whether a given system can be stabilized using energy shaping feedback. Most of the previous results have dealt with a particular solution and neither the role of closed-loop stability nor a complete exploration of the space of solutions has been discussed with any degree of generality. We use the geometric theory of partial differential equations originated by Goldschmidt and Spencer in the late 1960's using jet bundle structure [31, 18, 34]. We describe the energy shaping partial differential equations as a fibered submanifold of the $k$-jet bundle (in our case, $k=1$ ) of a fibered manifold. By revealing the geometric structure of kinetic energy shaping, we observe similarities of the problem of kinetic energy shaping with some well-known problems in Riemannian geometry; in particular, the problem of finding a metric connection, initiated by Eisenhart and Veblen [16].

We also discuss the integrability of the $\lambda$-equation from a geometric point of view $[18,19]$, and we address some interesting geometric features of the integrability 
conditions in the $\lambda$-method. In particular, we notice that the necessary conditions for the set of $\lambda$-equations restricted to the underactuated distribution are related to the Ricci identity [12, 23], an identity which also features prominently in the work of Eisenhart and Veblen [16] on the metric connection problem. The similarities of the kinetic energy shaping problem and the metric connection problem, we reveal the geometric structure of the compatibility conditions.

We also couple the set of $\lambda$-equations for kinetic energy shaping with the integrability results of potential energy shaping [25]. This allows us to address some key questions in energy shaping that have not been addressed to this point. For example, the procedure shows how a poor design of closed-loop metric can make it impossible to achieve any flexibility in the character of the possible closed-loop potential functions. Finally, we discuss systems with one degree of underactuation and we show that for this class of systems there is always a solution to the potential energy shaping problem for each closed-loop energy shaping metric.

This paper is organized as follows. In Section 2 we review the affine geometric setup for the energy shaping problem [24], and we state some of the fundamental open problems in energy shaping. In Section 3 we give a brief review of the mathematical structures we use in this paper. In particular, Section 3.1 gives an introduction to the geometric methods for analyzing formal integrability of partial differential equations [18], [19]. The main character of the theorems in this section is extremely algebraic and may seem unmotivated to a reader unfamiliar with the formal theory of partial differential equations. A reader new to these techniques is advised that some effort will be required to become comfortable with them. In Section 3.2 we motivate the definition of a connection as a section of a jet bundle [30] in order to give a precise definition for the space of torsion free connections on a manifold. We give a geometric formulation for the partial differential equations of the kinetic energy shaping in Section 4, and we recall the existing results for potential energy shaping [25]. In this paper we use the geometric formulation of the kinetic energy shaping problem using the $\lambda$-method [5], [13]. We review and reprove the main results of the $\lambda$-method in Section 4.2. Section 5 contains the main contribution of the paper. We prove that the set of $\lambda$-equations has an involutive symbol and is formally integrable under a certain surjectivity condition. In other words, we give sufficient conditions for the existence of a formal solution to the $\lambda$-equations. Section 7 deals with the potential energy shaping problem. We analyze the set of conditions in [25] to characterize the set of acceptable closed-loop metrics. Finally, in Section 8 we give a set of sufficient conditions for total energy shaping and, as an example, we specialize our results to systems with one degree of underactuation. In particular we show that in this case, for any closed-loop metric that satisfies the kinetic energy shaping conditions a closed-loop potential energy shaping is achievable. 
Notation. The basic differential geometric notations that we use in this paper are those of [1] and [11]. The identity map for a set $S$ is denoted by $\operatorname{id}_{S}$ and the image of a map $f: S \rightarrow W$ by $\operatorname{Im}(f)$. For a vector space $\mathrm{V}$ the set of $(r, s)$-tensors on $\mathrm{V}$ is denoted by $\mathrm{T}_{s}^{r}(\mathrm{~V})$. By $\mathrm{S}_{k} \mathrm{~V}$ and $\Lambda_{k} \mathrm{~V}$ we denote, respectively, the set of symmetric and skew-symmetric $(0, k)$-tensors on $\mathrm{V}$. We shall also require symmetrizing and skew-symmetrizing maps. Thus, for $\mathrm{A} \in \mathrm{T}_{k}^{0}(\mathrm{~V})$, we define the following projection maps:

$$
\begin{gathered}
\operatorname{Alt}(\mathrm{A})\left(v_{1}, \cdots, v_{k}\right)=\frac{1}{k !} \sum_{\sigma \in \mathfrak{S}_{k}}(-1)^{\operatorname{sgn}(\sigma)} \mathrm{A}\left(v_{\sigma(1)}, \cdots, v_{\sigma(k)}\right) ; \\
\operatorname{Sym}(\mathrm{A})\left(v_{1}, \cdots, v_{k}\right)=\frac{1}{k !} \sum_{\sigma \in \mathfrak{S}_{k}} \mathrm{~A}\left(v_{\sigma(1)}, \cdots, v_{\sigma(k)}\right),
\end{gathered}
$$

where $\mathfrak{S}_{k}$ is the permutation group on $k$ symbols and $\operatorname{sgn}(\sigma)$ is the parity of the permutation $\sigma$. Let $\mathrm{A}$ be a $(0,2)$-tensor on $\mathrm{V}$. We define the flat map $\mathrm{A}^{\mathrm{b}}: \mathrm{V} \rightarrow \mathrm{V}^{*}$ by $\left\langle\mathrm{A}^{b}(u) ; v\right\rangle=\mathrm{A}(u, v), u, v \in \mathrm{V}$. The inverse of the flat map is denoted by $\mathrm{A}^{\sharp}: \mathrm{V}^{*} \rightarrow \mathrm{V}$ in case $A^{b}$ is invertible. We also define a similar notation for a $(0,3)$-tensor $A$ on $V$ by

$$
\left\langle\mathrm{A}^{b}(u), w\right\rangle=\mathrm{A}(w, u, u), \quad u, w \in \mathrm{V} .
$$

For $S \subset \mathrm{V}$ and $W \subset \mathrm{V}^{*}$ we denote

$$
\begin{aligned}
\operatorname{ann}(S) & =\left\{\alpha \in \mathrm{V}^{*} \mid \alpha(v)=0, \forall v \in S\right\}, \\
\operatorname{coann}(W) & =\left\{v \in \mathrm{V}^{*} \mid \alpha(v)=0, \forall \alpha \in W\right\} .
\end{aligned}
$$

For the purpose of using a version of the Cartan-Kähler theorem, all manifolds and maps will be assumed to be analytic unless otherwise stated. Many of the theorems and lemmas are still true in the smooth case. Let $Q$ be an analytic manifold, if $\pi: E \rightarrow Q$ is an analytic vector bundle, $\Gamma^{\omega}(\mathrm{E})$ denotes the set of analytic sections of $E$. We denote the tangent bundle of $Q$ by $\pi_{Q}: T Q \rightarrow Q$. The set of analytic functions on $\mathrm{Q}$ is denoted by $C^{\omega}(\mathrm{Q})$. The exterior derivative of a $k$-form $\alpha$ on $\mathrm{Q}$ is denoted by $\mathrm{d} \alpha$. For a $(0, k)$-tensor field $\mathrm{A}$ and a Riemannian metric $\mathbb{G}$ on $\mathrm{Q}$, we define the $(1, k-1)$-tensor field $\mathbb{G}^{\sharp} \mathrm{A}$ by

$$
\mathbb{G}^{\sharp} \mathrm{A}\left(\alpha, X_{1}, \cdots, X_{k-1}\right)=\mathrm{A}\left(\mathbb{G}^{\sharp}(\alpha), X_{1}, \cdots, X_{k-1}\right),
$$

where $\alpha \in \Gamma^{\omega}\left(\mathrm{T}^{*} \mathrm{Q}\right), X_{1}, \cdots, X_{k} \in \Gamma^{\omega}(\mathrm{TQ})$. Finally, we give a decomposition of the $(0,3)$-tensor fields. We call a $(0,3)$-tensor field $\mathrm{A}$ on $\mathrm{Q}$ :

a) gyroscopic if $\mathrm{A}\left(X_{1}, X_{2}, X_{3}\right)=-\mathrm{A}\left(X_{2}, X_{1}, X_{3}\right), \quad \forall X_{1}, X_{2}, X_{3} \in \Gamma^{\omega}(\mathrm{TQ})$;

b) torsional if $\mathrm{A}\left(X_{1}, X_{2}, X_{3}\right)=-\mathrm{A}\left(X_{1}, X_{3}, X_{2}\right), \quad \forall X_{1}, X_{2}, X_{3} \in \Gamma^{\omega}(\mathrm{TQ})$;

c) geodesic if $\mathrm{A}\left(X_{1}, X_{2}, X_{3}\right)=\mathrm{A}\left(X_{1}, X_{3}, X_{2}\right), \quad \forall X_{1}, X_{2}, X_{3} \in \Gamma^{\omega}(\mathrm{TQ})$; 
d) skew if $A \in \Gamma^{\omega}\left(\Lambda_{3}(T Q)\right)$.

We denote the set of gyroscopic and torsional tensor fields on $Q$, respectively, by $\operatorname{Gyr}(T Q)$ and $\operatorname{Tor}(T Q)$. We can record the decomposition of $T_{3}^{0}(T Q)$ as follows [24], [17]:

$$
\mathrm{T}_{3}^{0}(\mathrm{TQ})=\mathrm{S}_{3}(\mathrm{TQ}) \oplus(\operatorname{Gyr}(\mathrm{TQ}) \cap \operatorname{ker} \mathrm{Alt}) \oplus(\operatorname{Tor}(\mathrm{TQ}) \cap \operatorname{ker} \mathrm{Alt}) \oplus \Lambda_{3}(\mathrm{TQ}) .
$$

2. Statement of the problem. A forced simple mechanical system is a quadruple $\Sigma=\left(\mathbb{Q}, \mathbb{G}, V, \mathcal{F}_{\mathrm{e}}\right)$ where $\mathrm{Q}$ is an $n$-dimensional manifold called the configuration manifold, $\mathbb{G}$ is a Riemannian metric on $Q, V$ is a function on the configuration manifold called the potential function and $\mathcal{F}_{\mathrm{e}}: \mathrm{TQ} \rightarrow \mathrm{T}^{*} \mathrm{Q}$ is a bundle map over $\operatorname{id}_{\mathrm{Q}}$ called the external force. We denote by $\nabla^{\mathbb{G}}$ the covariant derivative with respect to the associated Levi-Civita connection. The governing equations for a forced simple mechanical system are

$$
\nabla_{\gamma^{\prime}(t)}^{\mathbb{G}} \gamma^{\prime}(t)=-\mathbb{G}^{\sharp} \circ d V(\gamma(t))+\mathbb{G}^{\sharp} \mathcal{F}_{\mathrm{e}}\left(\gamma^{\prime}(t)\right),
$$

where $\gamma: I \rightarrow \mathrm{Q}$ is an analytic curve on $\mathbf{Q}$.

Similarly, a simple mechanical control system is a quintuple $\Sigma=\left(\mathrm{Q}, \mathbb{G}, V, \mathcal{F}_{\mathrm{e}}, \mathcal{W}\right)$ where $Q$ is an $n$-dimensional manifold called the configuration manifold, $\mathbb{G}$ is a Riemannian metric on $Q, V$ is a function on the configuration manifold called the potential function, $\mathcal{F}_{\mathrm{e}}: \mathrm{TQ} \rightarrow \mathrm{T}^{*} \mathrm{Q}$ is a bundle map over $\mathrm{id}_{\mathrm{Q}}$ called the external force and $\mathcal{W}$ is a subbundle of $T^{*} Q$ called the control subbundle [11]. The governing equations for a simple mechanical control system are

$$
\nabla_{\gamma^{\prime}(t)}^{\mathbb{G}} \gamma^{\prime}(t)=-\mathbb{G}^{\sharp} \circ d V(\gamma(t))+\mathbb{G}^{\sharp} \mathcal{F}_{\mathrm{e}}\left(\gamma^{\prime}(t)\right)+\mathbb{G}^{\sharp} u\left(\gamma^{\prime}(t)\right),
$$

where $\gamma: I \rightarrow \mathrm{Q}$ is a curve on $\mathrm{Q}$ and $u: \mathrm{TQ} \rightarrow \mathcal{W}$ is the control force. A class of external forces in which we are interested is gyroscopic forces.

Definition 2.1. Let $\Sigma=\left(\mathbb{Q}, \mathbb{G}, V, \mathcal{F}_{\mathrm{e}}\right)$ be a forced simple mechanical system. We call an external force $\mathcal{F}_{\mathrm{G}}: \mathrm{TQ} \rightarrow \mathrm{T}^{*} \mathrm{Q}$ a gyroscopic force if, for all $X \in \Gamma^{\omega}(\mathrm{TQ})$,

$$
\left\langle X, \mathcal{F}_{\mathrm{G}}(X)\right\rangle=0 .
$$

A linear gyroscopic force is a gyroscopic force with the following form:

$$
\mathcal{F}_{\mathrm{G}, 1}(X)=-\mathbb{B}_{\mathrm{G}, 1}^{b}(X), \quad X \in \Gamma^{\omega}\left(\mathrm{T}_{q} \mathrm{Q}\right)
$$

where $\mathbb{B}_{G, 1}$ is a skew-symmetric $(0,2)$-tensor. A quadratic gyroscopic force is a gyroscopic force $\mathcal{F}_{G, 2}$ with the following form:

$$
\mathcal{F}_{\mathrm{G}, 2}(X)=\mathbb{B}_{\mathrm{G}, 2}^{b}(X), \quad X \in \Gamma^{\omega}\left(\mathrm{T}_{q} \mathrm{Q}\right),
$$

where $\mathbb{B}_{\mathrm{G}, 2}$ is a $(0,3)$-tensor which is skew-symmetric in the first two arguments, i.e., $\mathbb{B}_{\mathrm{G}, 2}(X, Y, Z)=-\mathbb{B}_{\mathrm{G}, 2}(Y, X, Z), X, Y, Z \in \Gamma^{\omega}\left(\mathrm{T}_{q} \mathrm{Q}\right)$. By definition of the flat map, a 
quadratic gyroscopic force is defined by

$$
\left\langle\mathcal{F}_{\mathrm{G}, 2}(X) ; Z\right\rangle=\mathbb{B}_{\mathrm{G}, 2}(Z, X, X), \quad X, Z \in \Gamma^{\omega}\left(\mathrm{T}_{q} \mathrm{Q}\right)
$$

Given an open-loop simple mechanical control system $\Sigma_{\mathrm{ol}}=\left(\mathrm{Q}, \mathbb{G}_{\mathrm{ol}}, V_{\mathrm{ol}}, \mathcal{F}_{\mathrm{ol}}, \mathcal{W}_{\mathrm{ol}}\right)$, we seek a control force such that the closed-loop system is a forced simple mechanical system $\Sigma_{\mathrm{cl}}=\left(\mathrm{Q}, \mathbb{G}_{\mathrm{cl}}, V_{\mathrm{cl}}, \mathcal{F}_{\mathrm{cl}}\right)$, possibly with some external force. The reason for seeking this as the closed-loop system is that the stability analysis of the equilibria for mechanical systems is well understood [11, Chapter 6]. The class of gyroscopic forces does not change the total energy of the closed-loop system, while adding gyroscopic forces increases the possibility of finding a stable closed-loop system [13]. In this paper we assume that the open-loop external force $\mathcal{F}_{\text {ol }}$ is zero. Moreover, it turns out that only the quadratic gyroscopic forces are useful in extending the space of possible closed-loop metrics [24]. The objective, therefore, can be phrased with the following definition.

Definition 2.2. Let $\Sigma_{\mathrm{ol}}=\left(\mathrm{Q}, \mathbb{G}_{\mathrm{ol}}, V_{\mathrm{ol}}, \mathcal{F}_{\mathrm{ol}}, \mathcal{W}_{\mathrm{ol}}\right)$ be an open-loop simple mechanical control system with $\mathcal{F}_{\mathrm{ol}}=0$. If there exists a bundle map $u_{\mathrm{shp}}: \mathrm{TQ} \rightarrow \mathcal{W}_{\mathrm{ol}}$ (called control) with $u_{\mathrm{shp}}=-u_{\mathrm{kin}}-u_{\mathrm{pot}}$ such that the closed-loop system is a forced simple mechanical system $\Sigma_{\mathrm{cl}}=\left(\mathrm{Q}, \mathbb{G}_{\mathrm{cl}}, V_{\mathrm{cl}}, \mathcal{F}_{\mathrm{cl}}\right)$, where $\mathcal{F}_{\mathrm{cl}}$ is a quadratic gyroscopic force with associated $(0,3)$-tensor $\mathbb{B}$ and

$$
\begin{aligned}
& \text { 1. } \mathbb{G}_{\mathrm{ol}}^{\sharp} \circ u_{\text {kin }}\left(\gamma^{\prime}(t)\right)=\nabla_{\gamma^{\prime}(t)}^{\mathbb{G}_{\mathrm{cl}}} \gamma^{\prime}(t)-\nabla_{\gamma^{\prime}(t)}^{\mathbb{G}_{\mathrm{ol}}} \gamma^{\prime}(t)-\mathbb{G}_{\mathrm{cl}}^{\sharp} \circ\left(\mathbb{B}^{\mathrm{b}}\left(\gamma^{\prime}(t)\right)\right), \\
& \text { 2. } u_{\mathrm{pot}}(\gamma(t))=\mathbb{G}_{o l}^{b} \circ \mathbb{G}_{c l}^{\sharp} d V_{\mathrm{cl}}(\gamma(t))-d V_{\mathrm{ol}}(\gamma(t)),
\end{aligned}
$$

then the control $u_{\text {shp }}$ is called an energy shaping feedback.

REMARK 2.3. Throughout this work, we assume that the equilibrium point $q_{0} \in \mathrm{Q}$ is a regular point for $\mathcal{W}_{\mathrm{ol}}$. Moreover, we assume that the control codistribution $\mathcal{W}_{\mathrm{ol}}$ is integrable. This assumption is common in the literature and many examples fall into this case.

The conditions of Definition 2.2 contain as unknowns the closed-loop metric $\mathbb{G}_{\mathrm{cl}}$, the closed-loop potential energy $V_{\mathrm{cl}}$ and the gyroscopic $(0,3)$-tensor field $\mathbb{B}$. One can observe that these equations involve the first jet of the unknowns. One can construct concretely a set of first-order partial differential equations as necessary and sufficient conditions for the existence of an energy shaping feedback. Let $\mathcal{W} \subset T^{*} Q$ be a given subbundle and define the associated $\mathbb{G}_{\mathrm{ol}}$-orthogonal projection map $P \in \Gamma^{\omega}\left(\mathrm{T}^{*} \mathrm{Q} \otimes \mathrm{TQ}\right)$ by

$$
\operatorname{Ker}(P)=\mathbb{G}_{\mathrm{ol}}^{\sharp} \mathcal{W}
$$

Note that $P$ completely prescribes $\mathcal{W}$. We apply $P$ to the equation from part 1 of Definition 2.2 to arrive at the following equation:

$$
P\left(\nabla_{\gamma^{\prime}(t)}^{\mathbb{G}_{c l}} \gamma^{\prime}(t)-\nabla_{\gamma^{\prime}(t)}^{\mathbb{G}_{\mathrm{ol}}} \gamma^{\prime}(t)-\mathbb{G}_{\mathrm{cl}}^{\sharp} \circ \mathbb{B}^{b}\left(\gamma^{\prime}(t)\right)\right)=0 .
$$


Assume $\mathrm{Q}$ is an $n$-dimensional manifold and $\mathcal{W}$ is an integrable codistribution of dimension $n-m$. In adapted local coordinates the kinetic energy shaping partial differential equation is given by

$P_{r}^{a}\left(\mathbb{G}_{\mathrm{cl}}^{r l}\left(\mathbb{G}_{\mathrm{cl}, l j, k}+\mathbb{G}_{\mathrm{cl} l, l k, j}-\mathbb{G}_{\mathrm{cl}, k j, l}\right)-\mathbb{G}_{\mathrm{ol}}^{r l}\left(\mathbb{G}_{\mathrm{ol}, l j, k}+\mathbb{G}_{\mathrm{ol}, l k, j}-\mathbb{G}_{\mathrm{ol}, k j, l}\right)-\mathbb{G}_{\mathrm{cl}}^{r l} \mathbb{B}_{l k j}\right)=0$, where $i, j, k, l, r \in\{1, \cdots, n\}, a \in\{1, \cdots, m\}$ and we denote the first derivative of $\mathbb{G}_{\mathrm{cl} l j}$ with respect to $q^{k}$ by $\mathbb{G}_{\mathrm{cl}, l j, k}$. Similarly, let $\hat{P}: \mathrm{T}^{*} \mathrm{Q} \rightarrow \mathrm{T}^{*} \mathrm{Q} / \mathcal{W}_{\mathrm{ol}}$ be the canonical projection on to the quotient vector bundle. We have

$$
\hat{P}\left(\mathbb{G}_{\mathrm{ol}}^{b} \circ \mathbb{G}_{\mathrm{cl}}^{\sharp} d V_{\mathrm{cl}}(\gamma(t))-d V_{\mathrm{ol}}(\gamma(t))\right)=0 .
$$

In local coordinates we have

$$
\hat{P}_{a}^{i}\left(\mathbb{G}_{\mathrm{ol}, i j} \mathbb{G}_{\mathrm{cl}}{ }^{j k} V_{\mathrm{cl}, k}-V_{\mathrm{ol}, k}\right)=0,
$$

where $i, j, k \in\{1, \cdots, n\}, a \in\{1, \cdots, m\}$ and we denoted the first derivative of $V_{\mathrm{cl}}$ with respect to $q^{k}$ by $V_{\mathrm{cl}, k}$. For more details on the affine differential geometric setup of energy shaping problem see [24].

Now that the energy shaping partial differential equations have been specified, we provide a summary of some of the fundamental questions one can now ask.

\section{Some problems in energy shaping}

P1. Describe the set of achievable closed-loop metrics. There has not been much treatment on this problem in the literature apart from giving a geometric description of the problem [5].

P2. Assume that one has found a closed-loop metric which satisfies the kinetic energy shaping problem. What are the conditions under which there exists a closed-loop potential function which satisfies the potential energy shaping problem?

P3. Describe the set of achievable closed-loop potential functions by allowing the closed-loop metric to vary over the achievable set.

P4. Give a complete description of the set of stabilizing potential energy shaping functions. In order to have a stabilizing energy shaping feedback, the Hessian of the closed-loop potential functions should be positive definite. The type of obstruction this condition puts on the set of achieved energy shaping feedbacks has not yet been characterized in a geometric fashion.

P5. Describe the effect of including gyroscopic forces in the procedure of energy shaping. An algebraic presentation of this problem has been given in [24]. Although one can extend our results in the current paper to the case with gyroscopic forces, many geometric and algebraic constructions need to be performed to clarify how the results should be interpreted in terms of stabilization. 
P6. Reconstruct some of the existing results using the sufficient conditions presented in the current paper; namely, answer the following questions using the results of this paper:

(a) why is it always the case that one can construct an explicit solution to the set of partial differential equations for systems with one degree of underactuation?

(b) why is linear controllability a sufficient condition for existence of a stabilizing energy shaping feedback in linear systems?

P7. Find some interesting counterexamples. It would be revealing to have an example for which there exists no stabilizing energy shaping feedback, even under the absence of gyroscopic forces. This might help to understand the key primary question in energy shaping: when is it possible to stabilize a system by the energy shaping method?

\section{Answers in this paper}

A1. In Section 5 we partially answer Problem 1. Assuming that $\mathcal{W}_{\mathrm{ol}}$ is integrable, we describe a set of sufficient conditions under which one can construct a formal solution to the set of kinetic energy shaping problem in the analytic case and in the absence of gyroscopic forces. Moreover, we show that any analytic solution to the kinetic energy shaping problem satisfies those conditions. (See Theorems 5.6 and 6.6.)

A2. Lewis [25] presented a set of sufficient condition for Problem 2 using a geometric analysis of the potential energy shaping partial differential equations. In Section 7 we couple this sufficient condition with the kinetic energy shaping results. In other words, we give conditions on the closed-loop metric so that there exists a solution to the set of potential energy shaping partial differential equations. (See Theorem 7.8.)

A3. Problem 3 is wide open and even a clear geometric formulation of this problem is far from being achieved. In this paper, we start down one possible avenue by placing the problem in the setting of geometric partial differential equations [18], [19]. In particular, we give a set of conditions on the set of closedloop metrics under which there exists a closed-loop potential function that satisfies the set of potential energy shaping partial differential equations. (See Theorem 7.8.)

A6. Problem 6(a) has been discussed in [4] and [2]. But the results do not reveal how the geometric obstructions given by the kinetic and potential energy shaping conditions are satisfied. In Example 7.9 we give a result which essentially solves the problem. The second question has been posed and solved in [40]. It would be interesting to recover the sufficient conditions solely by looking at the integrability conditions for the energy shaping partial differential equations. 
3. Preliminaries. We review some basic background for modeling the system of partial differential equations in the energy shaping problem. This section consists of three main parts. The first part deals with the geometric modeling of partial differential equations and the second part gives a useful definition of a connection on a vector bundle which characterizes the structure of the set of all connections. Finally, we present the so-called Ricci identity [12] which plays a significant role in answering some questions about the kinetic energy shaping problem.

3.1. Formal integrability of partial differential equations. In this section, we describe the main technique that we use for studying the energy shaping partial differential equations. The discussion centers around an analogue for the Cauchy-Kowalevski theorem [10] and formal integrability. We follow the contributions made by Goldschmidt and Spencer in the late 1960's [18, 19, 34].

Although understanding the proofs of the main theorems depends on the techniques described in this section, we emphasize that the statement of the main results of the paper are accessible without understanding formal methods in detail. The main results in the paper involve applications of the important Theorem 3.20 stated below. However, the verification of the hypothesis of this theorem typically takes some effort. In this section we describe the tools used to verify the hypothesis of Theorem 3.20.

3.1.1. Representation of a partial differential equation as a fibered submanifold of a jet bundle. We denote by $(\mathrm{E}, \pi, \mathrm{Q})$ a fibered manifold $\pi: \mathrm{E} \rightarrow \mathrm{Q}$. The vertical bundle of a fibered manifold $\pi$ is the subbundle of $\mathrm{T} \pi$ given by $\mathrm{V} \pi=\operatorname{ker}(\mathrm{T} \pi)$. We denote by $\mathrm{J}_{k} \pi$ the bundle of $k$-jets [31]. If $(\xi, U)$ is an analytic local section of $\pi$, we denote its $k$-jet by $j_{k} \xi$. We denote an element of $\mathrm{J}_{k} \pi$ by $j_{k} \xi(x)$. If we represent the sheaf of germs of sections of $\pi$ by $\mathscr{S}_{\mathrm{Q}}(\pi)$, then $j_{k}$ induces a morphism of sheaves $\mathscr{S}_{\mathrm{Q}}(\pi) \rightarrow \mathscr{S}_{\mathrm{Q}}\left(\mathrm{J}_{k} \pi\right)$. We let $\pi_{k}: \mathrm{J}_{k} \pi \rightarrow \mathrm{Q}$ and $\pi_{l}^{k}: \mathrm{J}_{k} \pi \rightarrow \mathrm{J}_{l} \pi, l \leq k$, be the canonical projections. One can show that $\pi_{k}$ and $\pi_{l}^{k}$ are surjective submersions; moreover, $\pi_{l}^{k}: \mathrm{J}_{k} \pi \rightarrow \mathrm{J}_{l} \pi$ is an epimorphism of fibered manifolds and $\left(\mathrm{J}_{k} \pi, \pi_{l}^{k}, \mathrm{~J}_{l} \pi\right)$ is a bundle. The following definition establishes the relationship between jet bundles and systems of partial differential equations.

Definition 3.1. Let $(\mathrm{E}, \pi, \mathrm{Q})$ be a fibered manifold and let $\mathrm{J}_{k} \pi$ be its bundle of $k$-jets. A partial differential equation is a fibered submanifold $\mathrm{R}_{k} \subset \mathrm{J}_{k} \pi$.

We denote by $\hat{\pi}_{k}$ the restriction of $\pi_{k}$ to $\mathrm{R}_{k}$. As one can see, the "equation" representation of the partial differential equation is obscure here. The following local characterization of a partial differential equation as a kernel of a fibered manifold morphism is helpful in clarifying the equation point of view.

Proposition 3.2. Let $(\mathrm{E}, \pi, \mathrm{Q})$ be a fibered manifold. Given a partial differential equations $\mathrm{R}_{k} \subset \mathrm{J}_{k} \pi$ and a point $p \in \mathrm{Q}$, there exists neighborhood $U$ of $p$, a fibered manifold $\left(\mathrm{E}^{\prime}, \pi^{\prime}, U\right)$, an analytic section $\eta$ of $\pi^{\prime}$, and a morphism of fibered manifolds 
$\Phi: \pi_{k}^{-1}(U) \rightarrow \mathrm{E}^{\prime}$ such that

$$
\pi_{k}^{-1}(U) \cap \mathrm{R}_{k} \doteq \operatorname{ker}_{\eta} \Phi=\left\{u_{k} \in \pi_{k}^{-1}\left(u_{k}\right) \mid \Phi\left(u_{k}\right)=\eta\left(\pi_{k}\left(u_{k}\right)\right)\right\} .
$$

Proof. Because $\mathrm{R}_{k}$ is a fibered submanifold, there exists an adapted chart $\left(\mathcal{U}_{k}, \phi_{k}\right)$ for $\mathrm{J}_{k} \pi$ with the induced chart $(U, \phi)$ on $\mathrm{Q}$ such that

$$
\phi_{k}\left(\mathcal{U}_{k}\right) \subset \phi(U) \times V \times W \subset \mathbb{R}^{n} \times \mathbb{R}^{m} \times \mathbb{R}^{m^{\prime}} \quad n, m, m^{\prime} \in \mathbb{Z}_{\geq 0}
$$

and such that

$$
\pi_{k}^{-1}(U) \cap \mathrm{R}_{k}=\{(x, v, 0) \mid x \in \phi(U), v \in V\} .
$$

Take $\mathrm{E}^{\prime}=U \times V$ and $\pi^{\prime}(x, v)=x$. Taking $\Phi(u)=(x, v)$ and $\eta(x)=(x, 0)$, the result follows.

A morphism $\Phi: J_{k} \pi \rightarrow \pi^{\prime}$ of fibered manifolds induces a differential operator $\mathfrak{D}$ of order $k$ which is a sheaf morphism of the form $\Phi \circ j_{k}: \mathscr{S}_{\mathrm{Q}}(\mathrm{E}) \rightarrow \mathscr{S}_{\mathrm{Q}}\left(\mathrm{E}^{\prime}\right)$.

\subsubsection{Prolongations and symbols.}

Prolongation. The process of differentiating a partial differential equation in order to arrive at a higher order partial differential equation is called prolongation. One can phrase this statement as the following definition.

Definition 3.3. Let $(\mathrm{E}, \pi, \mathrm{Q})$ be a fibered manifold and let $\mathrm{R}_{k} \subset \mathrm{J}_{k} \pi$ be a partial differential equation. The $r^{\text {th }}$-prolongation of $\mathrm{R}_{k}$ is the subset

$$
\rho_{r}\left(\mathrm{R}_{k}\right)=\mathrm{J}_{r} \hat{\pi}_{k} \cap \mathrm{J}_{k+r} \pi .
$$

A partial differential equation $\mathrm{R}_{k}$ is regular if $\rho_{r}\left(\mathrm{R}_{k}\right)$ is a fibered submanifold of $\mathrm{J}_{k+r} \pi$ for each $r \in \mathbb{Z}_{\geq 0}$. One can represent the $r^{t h}$-prolongation of a partial differential equations using the associated morphism. The $r^{t h}$-prolongation of $\Phi$ is defined to be the unique morphism of fibered manifolds over $\mathrm{Q}, \rho_{r}(\Phi): \mathrm{J}_{r+k} \pi \rightarrow \mathrm{J}_{r} \pi^{\prime}$, that makes the following diagram commutes:

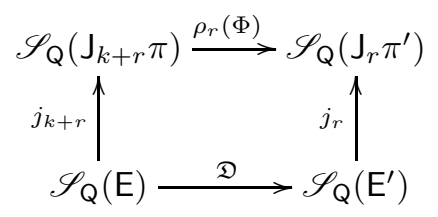

It is fairly clear that that for $r, l \in \mathbb{Z}_{\geq 0}$ and $r \geq l$ we have $\pi_{k+l}^{k+r}\left(\rho_{r}\left(\mathrm{R}_{k}\right)\right) \subset$ $\rho_{l}\left(\mathrm{R}_{k}\right)$. We adopt the notation $\hat{\pi}_{k+l}^{k+r}: \rho_{r}\left(\mathrm{R}_{k}\right) \rightarrow \rho_{l}\left(\mathrm{R}_{k}\right)$ and $\hat{\pi}_{k+r}: \rho_{r}\left(\mathrm{R}_{k}\right) \rightarrow \mathrm{Q}$ as the canonical projections. There is no guarantee that the first map is a surjective submersion; surjectivity of this map leads to the concept of formal integrability which will be discussed later. The following remark is advantageous for later purposes; for details of the proof we refer to [20], [19]. 
REMARK 3.4. Let $\pi$ be a fibered manifold as before and let $\mathrm{R}_{k} \subset \mathrm{J}_{k} \pi$ be a partial differential equation. If $\rho_{r}\left(\mathrm{R}_{k}\right)$ is a fibered submanifold of $\mathrm{J}_{k+r} \pi$, then $\rho_{l}\left(\rho_{r}\left(\mathrm{R}_{k}\right)\right)=$ $\rho_{l+r}\left(\mathrm{R}_{k}\right)$. Since one can define $\rho_{r}\left(\mathrm{R}_{k}\right)$ as the kernel of a morphism of fibered manifolds, this follows immediately from studying the following exact commutative diagram:

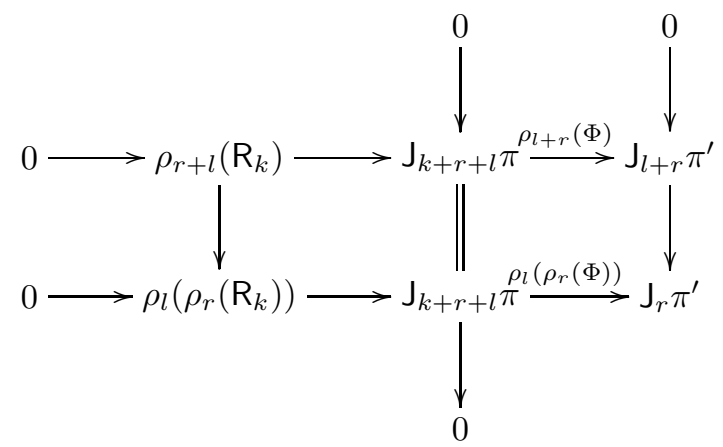

Whenever $\mathrm{R}_{k}$ is regular, for sake of convenience, we use $\mathrm{R}_{k+r}$ for the $r^{\text {th }}$-prolongation.

Symbols. The highest order terms in the linearization of a partial differential equation carry valuable information about formal integrability of the partial differential equation [18]. Similar to our approach for defining a partial differential equation, we give two equivalent formal definitions to capture these higher order terms, one as a vector bundle morphism and one as a family of subspaces.

Given $p_{k-1} \in \mathrm{J}_{k-1} \pi$, recall that $\left(\pi_{k-1}^{k}\right)^{-1}\left(p_{k-1}\right)$ has the structure of an affine space modeled on $\mathrm{S}_{k} \mathrm{~T}_{\pi_{k-1}\left(p_{k-1}\right)} \mathrm{Q} \otimes \mathrm{V}_{\pi_{0}^{k-1}\left(p_{k-1}\right)} \pi$. For each $p_{k} \in \mathrm{J}_{k} \pi$ we have $\mathrm{V}_{p_{k}} \pi_{k-1}^{k}$ $\simeq \mathrm{S}_{k} \mathrm{~T}_{\pi_{k-1}\left(p_{k-1}\right)}^{*} \mathrm{Q} \otimes \mathrm{V}_{\pi_{0}^{k-1}\left(p_{k-1}\right)} \pi$ as well as a vector bundle isomorphism $\pi_{k}^{*} \mathrm{~S}_{k} \mathrm{~T}^{*} \mathrm{Q} \otimes$ $\left(\pi_{0}^{k}\right)^{*} \mathrm{~V} \pi \cong \mathrm{V} \pi_{k-1}^{k}$. The identification of these bundles is made implicitly in most of the literature [31] and we follow this convention.

Definition 3.5. Let $(\mathrm{E}, \pi, \mathrm{Q})$ be a fibered manifold and let $\mathrm{R}_{k} \subset \mathrm{J}_{k} \pi$ be a partial differential equation. The symbol of $\mathrm{R}_{k}$ is the family $\mathrm{G}_{k}$ of vector spaces given by

$$
\left.\mathrm{G}_{k}\right|_{p_{k}}=\mathrm{V}_{p_{k}} \hat{\pi}_{k} \cap \mathrm{V}_{p_{k}} \pi_{k-1}^{k}, \quad p_{k} \in \mathrm{J}_{k} \pi .
$$

Let $\xi$ be a section of $\mathrm{E}$ over an open neighborhood $U \subset \mathrm{Q}$ and let $p \in U$. Let $\left\{f_{1}, \cdots, f_{n}\right\}$ be $\mathbb{R}$-valued functions defined on a neighborhood $U$ of $p \in \mathrm{Q}$ which vanish at $p$. Define $\epsilon_{k}: \mathrm{S}_{k} \mathrm{~T}^{*} \mathrm{Q} \otimes V \pi \rightarrow \mathrm{V} \pi_{k}$ by [18]

$$
\epsilon_{k}:\left(d f_{1} \cdots d f_{k} \otimes \xi\right)(p) \rightarrow j_{k}\left(\left(\Pi_{i=1}^{k} f_{i}\right) \cdot \xi\right)(p) .
$$

$\epsilon_{k}$ is well-defined since the derivatives of $\left(\Pi_{i=1}^{k} f_{i}\right)$ vanish up to order $k-1$ at $p$. We have the following lemma.

LEMMA 3.6. Let $(\mathrm{E}, \pi, \mathrm{Q})$ be a fibered manifold. We have the following short exact sequence of vector bundles over $\mathrm{J}_{k} \pi$ :

$$
0 \longrightarrow \mathrm{S}_{k} \mathrm{~T}^{*} \mathrm{Q} \otimes \mathrm{V} \pi \stackrel{\epsilon_{k}}{\longrightarrow} \mathrm{V} \pi_{k} \stackrel{\mathrm{V} \pi_{k-1}^{k}}{\longrightarrow}\left(\pi_{k-1}^{k}\right)^{*}\left(\mathrm{~V} \pi_{k-1}\right) \longrightarrow 0
$$


The following definition introduces the symbol map as a morphism of vector bundles. It is crucial to understand the distinction between the definition of the symbol map as a bundle map and the definition of the symbol map at a point as a map of vector spaces. This explicit distinction is usually dropped in the literature.

Definition 3.7. Let $(\mathrm{E}, \pi, \mathrm{Q})$ and $\left(\mathrm{E}^{\prime}, \pi^{\prime}, \mathrm{Q}\right)$ be fibered manifolds and let $\Phi$ : $\mathrm{J}_{k} \pi \rightarrow \mathrm{E}^{\prime}$ be a morphism over $\mathrm{id}_{\mathrm{Q}}$. The symbol of $\Phi$ is defined to be

$$
\sigma(\Phi)=\mathrm{V} \Phi \circ \epsilon_{k}: \pi_{k}^{*} \mathrm{~S}_{k} \mathrm{~T}^{*} \mathrm{Q} \otimes\left(\pi_{0}^{k}\right)^{*} \mathrm{~V} \pi \rightarrow \mathrm{V} \pi^{\prime}
$$

The following proposition relates the definition of the symbol as family of vector spaces with that as a map.

Proposition 3.8. Let $\pi$ be a fibered manifold as above and let $p_{k} \in \mathrm{R}_{k} \subset \mathrm{J}_{k} \pi$. Then the following sequences are exact:

1. $\left.0 \longrightarrow \mathrm{G}_{k}\right|_{p_{k}} \longrightarrow \mathrm{S}_{k} \mathrm{~T}_{\pi_{k}\left(p_{k}\right)}^{*} \mathrm{Q} \otimes \mathrm{V}_{\pi_{o}^{k}\left(p_{k}\right)} \stackrel{\left.\sigma(\Phi)\right|_{p_{k}}}{\longrightarrow} \mathrm{V}_{\Phi\left(p_{k}\right)} \pi^{\prime}$;

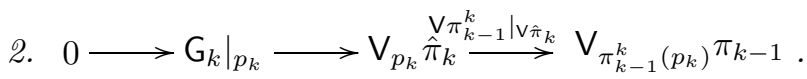

Proof. The proof of exactness of the first sequence follows from the following exact commutative diagram:

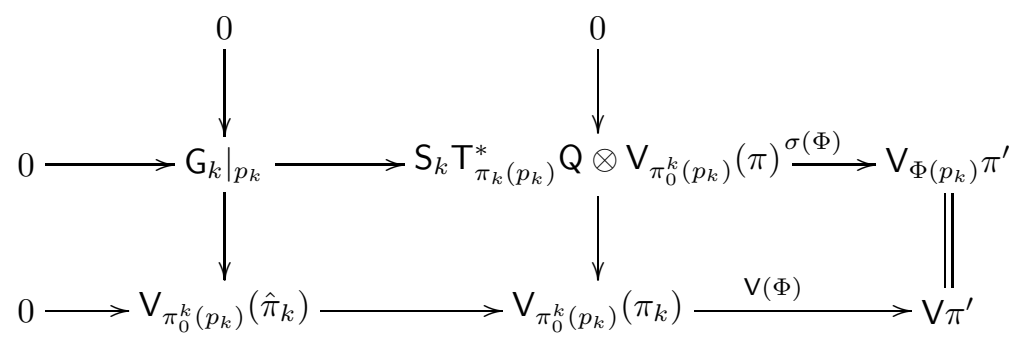

Similarly, for the second sequence, one should consider the following exact commutative diagram:

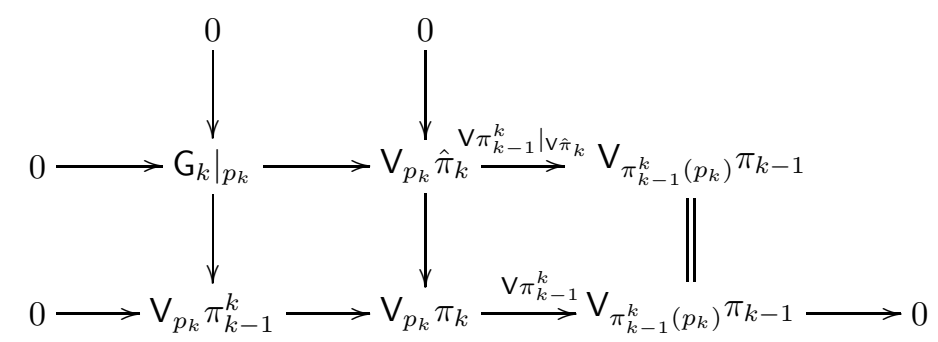

The second row is exact since $\mathrm{V} \pi_{k-1}^{k}$ is an epimorphism of vector spaces.

Note that $G_{k}$ is not always a vector bundle over $\vee \hat{\pi}_{k}$.

Prolongation of symbols. We establish a process for prolonging the symbol of a partial differential equation. This process can be obtained in a purely algebraic 
manner [21]. Let $(\mathrm{E}, \pi, \mathrm{Q})$ be a fibered manifold and $\mathrm{R}_{k} \subset \mathrm{J}_{k} \pi$ a partial differential equation. We fix a point $p_{k} \in \mathrm{R}_{k}$, we let $x=\pi_{k}\left(p_{k}\right)$ and we let $\left\{e^{1}, \cdots, e^{n}\right\}$ be a basis for $\mathrm{T}_{x}^{*} \mathrm{Q}$. We denote a basis element for $\mathrm{S}_{k} \mathrm{~T}_{x}^{*} \mathrm{Q}$ by $e^{i_{1}} e^{i_{2}} \cdots e^{i_{k}}$, where $i_{1}, \cdots, i_{k} \in$ $\{1, \cdots, n\}$ satisfy $i_{1} \leq i_{2} \leq \cdots \leq i_{n}$. For $k, r \in \mathbb{Z}_{\geq 0}$ we define the natural inclusion $\Delta_{k, r}: \mathrm{S}_{k+r} \mathrm{~T}_{x}^{*} \mathrm{Q} \rightarrow \mathrm{S}_{r} \mathrm{~T}_{x}^{*} \mathrm{Q} \otimes \mathrm{S}_{k} \mathrm{~T}_{x}^{*} \mathrm{Q}$ by

$$
\Delta_{k, r}: A_{i_{1} \cdots i_{k+r}} e^{i_{1}} e^{i_{2}} \cdots e^{i_{k+r}} \mapsto A_{i_{1} \cdots i_{r} i_{r+1} \cdots i_{k+r}} e^{i_{1}} e^{i_{2}} \cdots e^{i_{r}} \otimes e^{i_{r+1}} \cdots e^{i_{k+r}} .
$$

The map $\Delta_{k, r}$ can be extended naturally to

$$
\Delta_{k, r} \otimes \mathrm{id}_{\mathrm{V}_{\pi}}: \mathrm{S}_{k+r} \mathrm{~T}_{x}^{*} \mathrm{Q} \otimes \mathrm{V}_{\pi_{0}^{k}\left(p_{k}\right)} \pi \rightarrow \mathrm{S}_{k} \mathrm{~T}_{x}^{*} \mathrm{Q} \otimes \mathrm{S}_{r} \mathrm{~T}_{x}^{*} \mathrm{Q} \otimes \mathrm{V}_{\pi_{0}^{k}\left(p_{k}\right)} \pi
$$

Let $\Phi: \mathrm{J}_{k} \pi \rightarrow \pi^{\prime}$ be the local morphism associated to $\mathrm{R}_{k}$ and let $\sigma(\Phi)$ be the associated symbol map. With $\left.\mathrm{G}_{k}\right|_{p_{k}}=\left.\operatorname{ker} \sigma(\Phi)\right|_{p_{k}}$, we establish the $r^{t h}$-prolongation of the symbol by the following definition.

Definition 3.9. Let $\mathrm{R}_{k} \subset \mathrm{J}_{k} \pi$ be a partial differential equation. For each $p_{k} \in \mathrm{R}_{k}$ with $x=\pi_{k}\left(p_{k}\right)$, the map

$$
\rho_{r}\left(\left.\sigma(\Phi)\right|_{p_{k}}\right): \mathrm{S}_{k+r} \mathrm{~T}_{x}^{*} \mathrm{Q} \otimes \mathrm{V}_{\pi_{0}^{k}\left(p_{k}\right)} \pi \rightarrow \mathrm{S}_{r} \mathrm{~T}_{x}^{*} \mathrm{Q} \otimes \mathrm{V}_{\Phi\left(p_{k}\right)} \pi^{\prime}
$$

defined by $\left(\left.\operatorname{id}_{\mathrm{S}_{r} \mathrm{~T}_{x}^{*} \mathrm{Q}} \otimes \sigma(\Phi)\right|_{p_{k}}\right) \circ\left(\Delta_{k, r} \otimes \mathrm{id}_{V_{\pi}}\right)$ is called the $r^{\text {th }}$-prolongation of $\left.\sigma(\Phi)\right|_{p_{k}}$. Its kernel is denoted by $\rho_{r}\left(\left.\mathrm{G}_{k}\right|_{p_{k}}\right)$ and is called the $r^{\text {th }}$-prolongation of the symbol.

REMARK 3.10. Even if $\mathrm{G}_{k}$ is a vector bundle over $\mathrm{V} \hat{\pi}_{k}, \rho_{r}\left(\mathrm{G}_{k}\right)$ might not be a vector bundle over $\mathrm{V} \hat{\pi}_{k}$. In case it is, we sometimes use the notation $\mathrm{G}_{k+r}$ instead of $\rho_{r}\left(\mathrm{G}_{k}\right)$.

3.1.3. Formal integrability. Given a partial differential equation, we would like to study the existence of solutions. Specifically, we would like to construct the solutions of a given partial differential equation by constructing its Taylor series order by order. Since the theory we use rests on the Cauchy-Kowalevski theorem we assume analyticity of all the data. We start by giving a formal definition for solutions.

Definition 3.11. Let $(\mathrm{E}, \pi, \mathrm{Q})$ be a fibered manifold and let $\mathrm{R}_{k} \subset \mathrm{J}_{k} \pi$ be a $k$ th-order partial differential equation. A local formal solution of order $k$ is a pair $\left(\xi_{k}, U\right)$ where $U$ is an open subset of $\mathrm{Q}$ and $\xi_{k}$ is a section of $\mathrm{R}_{k}$ over $U$. If $\mathrm{R}_{k}$ is regular, one can define a formal solution of order $(k+r)$ as a pair $\left(\xi_{k+r}, U\right)$, where $\xi_{k+r}$ is a section of $\mathrm{R}_{k+r}$.

One can come up with different examples which are not "formally integrable" in the sense that one can not construct a solution as a Taylor series.

Definition 3.12. Let $(\mathrm{E}, \pi, \mathrm{Q})$ be a fibered manifold and let $\mathrm{R}_{k} \subset \mathrm{J}_{k} \pi$ be a regular partial differential equation. Then $\mathrm{R}_{k}$ is called formally integrable if the maps $\pi_{k+r}^{k+r+1}: \rho_{r+1}\left(\mathrm{R}_{k}\right) \rightarrow \rho_{r}\left(\mathrm{R}_{k}\right)$ are epimorphisms of fibered manifolds for each $r \in \mathbb{Z}_{\geq 0}$. 
Proposition 3.13. If $\mathrm{R}_{k}$ is formally integrable then $\rho_{r}\left(\mathrm{G}_{k}\right)$ is a vector bundle over $\mathrm{R}_{k}$ for each $r \in \mathbb{Z}_{\geq 0}$.

Proof. As $\mathrm{R}_{k}$ is formally integrable, $\pi_{k+r}^{k+r+1}$ is an epimorphism and so locally of constant rank. Then the following short exact sequence:

$$
0 \longrightarrow \mathrm{G}_{k+r+1} \longrightarrow \mathrm{V}\left(\hat{\pi}_{k+r+1}\right) \longrightarrow \mathrm{V}\left(\hat{\pi}_{k+r}\right) \longrightarrow 0
$$

yields that $\mathrm{G}_{k+r}$ is of constant rank.

The $\delta$-sequence. Another purely algebraic construction which is used extensively for the formal theory is the $\delta$-sequence. The $\delta$-sequence has been utilized by Spencer [33] in the theory of deformation of structures. We describe this construction in the partial differential equation framework, omitting some details, and we construct the $\delta$-sequence for $\mathrm{T}^{*} \mathrm{Q}$ which provides a characterization of the $\delta$ operator with the fiberwise exterior derivative on the set of differential $r$-forms on $\mathrm{T}^{*} \mathrm{Q}$. Generally, there is no necessity for a manifold structure and one can give the construction of the $\delta$-sequence in a purely algebraic fashion $[21]$.

We start by characterizing $\Lambda_{r} T^{*} \mathrm{Q} \otimes \mathrm{S}_{k} \mathrm{~T}^{*} \mathrm{Q}$ as a subset of differential $r$-forms on $T^{*} \mathrm{Q}$. First we give the following lemma whose proof is straightforward.

Lemma 3.14. Let $F$ be a $\mathbb{R}$-vector space and denote by

$$
P_{k}(F)=\left\{f: F \rightarrow \mathbb{R} \mid f(x)=\mathrm{A}(x, \cdots, x), \quad \mathrm{A} \in \mathrm{T}_{k}^{0}(F)\right\},
$$

the symmetric homogenous functions of degree $k$. Then, for $f \in P_{k}(F)$, there exists a unique $\mathrm{A} \in \mathrm{S}_{k}(F)$ such that $\mathrm{A}(x, \cdots, x)=f(x)$ for each $x \in F$.

Lemma 3.15. The following map from $\Lambda_{r} \mathrm{~T}_{x}^{*} \mathrm{Q} \otimes \mathrm{S}_{k} \mathrm{~T}_{x}^{*} \mathrm{Q}$ to the set of differential $r$-forms on $\mathrm{T}_{x}^{*} \mathrm{Q}$ is a monomorphism of $\mathbb{R}$-vector spaces:

$\phi_{k, r}(\alpha \otimes \mathrm{A})(x)\left(v_{1}, \cdots, v_{r}\right)=\mathrm{A}(x, \cdots, x) \alpha\left(v_{1}, \cdots, v_{r}\right), v_{1}, \cdots, v_{r} \in \mathrm{T}_{p} \mathbf{T}_{x}^{*} \mathrm{Q} \cong \mathrm{T}_{x}^{*} \mathrm{Q}$,

where $p \in \mathrm{T}_{x}^{*} \mathrm{Q}$.

The characterization basically identifies the symmetric tensor part of $\Lambda_{r} \mathrm{~T}_{x}^{*} \mathrm{Q} \otimes$ $\mathrm{S}_{k} \mathrm{~T}_{x}^{*} \mathrm{Q}$ with a homogenous polynomial function of order $k$. Let $\mathrm{d}_{r}$ be the exterior derivative on $\mathrm{T}_{x}^{*} \mathrm{Q}$ restricted to differential $r$-forms. One can define a linear map $\delta_{r, k}: \Lambda_{r} \mathrm{~T}_{x}^{*} \mathrm{Q} \otimes \mathrm{S}_{k} \mathrm{~T}_{x}^{*} \mathrm{Q} \rightarrow \Lambda_{r+1} \mathrm{~T}_{x}^{*} \mathrm{Q} \otimes \mathrm{S}_{k-1} \mathrm{~T}_{x}^{*} \mathrm{Q}$ by asking that the following diagram be commutative:

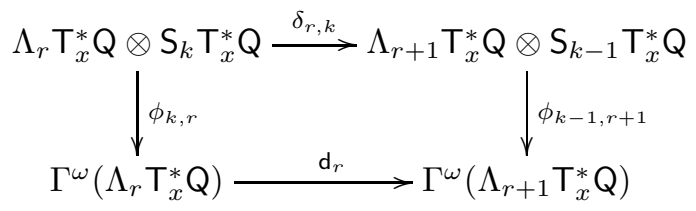


Explicitly, for $\alpha \in \Lambda_{r} \mathrm{~T}_{x}^{*} \mathrm{Q}$ and $\mathrm{A} \in \mathrm{S}_{k} \mathrm{~T}_{x}^{*} \mathrm{Q}$,

$$
\begin{aligned}
& \delta_{r, k}(\alpha \otimes \mathrm{A})\left(v_{1}, \cdots, v_{r+1}, u_{1}, \cdots, u_{k-1}\right)= \\
& \qquad \sum_{j=1}^{r+1} r \alpha\left(v_{1}, \cdots, \hat{v}_{j}, \cdots, v_{r+1}\right) \mathrm{A}\left(v_{j}, u_{1}, \cdots, u_{k-1}\right) .
\end{aligned}
$$

In other words, the $\delta_{r, k}$ operator imitates the exterior derivative on the space of differential forms on $\mathrm{T}_{x}^{*} \mathrm{Q}$ when we identify the symmetric homogenous polynomials of degree $k$ with a symmetric $k$-tensor.

It turns out that the following sequence, the so-called $r^{t h} \delta$-sequence, is exact (here we simply denote $\delta_{r, k}$ by $\delta$ ):

$$
\begin{aligned}
0 \longrightarrow \mathrm{S}_{n} \mathrm{~T}_{x}^{*} \mathrm{Q} \stackrel{\delta}{\longrightarrow} \mathrm{T}_{x}^{*} \mathrm{Q} \otimes \mathrm{S}_{n-1} \mathrm{~T}_{x}^{*} \mathrm{Q} \stackrel{\delta}{\longrightarrow} \cdots \\
\cdots \stackrel{\delta}{\longrightarrow} \Lambda_{r} \mathrm{~T}_{x}^{*} \mathrm{Q} \otimes \mathrm{S}_{n-r} \mathrm{~T}_{x}^{*} \mathrm{Q} \longrightarrow 0
\end{aligned}
$$

Let $\mathrm{R}_{k} \subset \mathrm{J}_{k} \pi$ be a partial differential equation. Consider the following exact and commutative diagram:

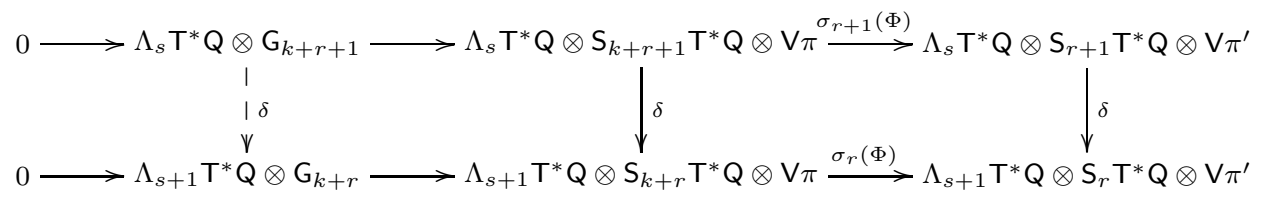

The map $\delta$ induces a new $\delta$-sequence for the symbol at each point. Note that sequences involving the symbol shall really be specified at each point and for the sake of simplicity we omit the point. What is more, there is no guarantee that this sequence is exact in general. Summarizing, we have the following graded differential complex:

$$
\begin{aligned}
0 \longrightarrow \mathrm{G}_{k+r} \stackrel{\delta}{\longrightarrow} \mathrm{T}^{*} \mathrm{Q} \otimes \mathrm{G}_{k+r-1} \stackrel{\delta}{\longrightarrow} \cdots \\
\cdots \stackrel{\delta}{\longrightarrow} \Lambda_{n} \mathrm{~T}^{*} \mathrm{Q} \otimes \mathrm{G}_{k+r-n} \longrightarrow 0 .
\end{aligned}
$$

We denote by $\mathrm{H}_{k+r-s}^{s}\left(\mathrm{G}_{k}\right)$ the cohomology at $\Lambda_{s} \mathrm{~T}^{*} \mathrm{Q} \otimes \mathrm{G}_{k+r-s}$ of this complex and we call it the Spencer cohomology group of degree $k+r-s$.

$$
\mathrm{H}_{k+r-s}^{s}\left(\mathrm{G}_{k}\right)=\operatorname{ker}\left(\delta_{s, k+r-s}\right) / \operatorname{Im}\left(\delta_{s-1, k+r+1-s}\right) .
$$

$\mathrm{G}_{k}$ is said to be $m$-acyclic if $\mathrm{H}_{k+r}^{s}=0$ for all $0 \leq s \leq m$ and $r \geq 0$.

Definition 3.16. Let $\mathrm{Q}$ be an n-dimensional manifold and let $\mathrm{R}_{k} \subset \mathrm{J}_{k} \pi$ be a partial differential equation as above. If the symbol is $n$-acyclic it is called involutive.

By definition, a symbol is involutive if and only if its corresponding $\delta$-sequences are exact. In particular, the symbol of the trivial system of partial differential equations is involutive.

REMARK 3.17. The concept of involutivity is a prominent algebraic concept which is not easy to grasp at first glance and it is simply not possible to provide a complete 
review of the concept in this document. Guillemin and Sternberg relate the different interpretations of an involutive symbol and actually propose a practical method for verifying involutivity [22]. J. P. Serre's complementary note on the appendix of this paper completes the picture by relating the sequence given in equation 2 to the Koszul complex.

We next address the concept of quasi-regular basis and a practical method for verifying involutivity [32].

Definition 3.18 (Quasi-regular basis). Let $(\mathrm{E}, \pi, \mathrm{Q})$ be a bundle with $\mathrm{Q}$ an ndimensional manifold and $x \in \mathrm{Q}$. Let $\mathrm{R}_{k} \subset \mathrm{J}_{k} \pi$ be a partial differential equation with associated symbol $\mathrm{G}_{k}$ and let $p_{k} \in \mathrm{R}_{k}$ be such that $\pi_{k}\left(p_{k}\right)=x$. A basis $\left\{\alpha^{1}, \cdots, \alpha^{n}\right\}$ for $\mathrm{T}_{x}^{*} \mathrm{Q}$ is called quasi-regular if

$$
\operatorname{dim}\left(\left.\mathrm{G}_{k+1}\right|_{p_{k+1}}\right)=\operatorname{dim}\left(\left.\mathrm{G}_{k}\right|_{p_{k}}\right)+\sum_{j=1}^{n-1} \operatorname{dim}\left(\left.\mathrm{G}_{k, j}\right|_{\left(x, p_{k}\right)}\right),
$$

where

$$
\left.\mathrm{G}_{k, j}\right|_{\left(x, p_{k}\right)}=\left.\left.\mathrm{G}_{k}\right|_{p_{k}} \cap \mathrm{S}_{k} \Sigma_{j}\right|_{x}
$$

and $\Sigma_{j}$ is the subspace of $\mathrm{T}_{x}^{*} \mathrm{Q}$ generated by $\left\{\alpha^{j+1}, \cdots, \alpha^{n}\right\}$.

The following theorem relates the concept of involutivity to the existence of a quasi-regular basis; the proof of the theorem can be found in [32].

TheOREm 3.19 (Criterion of involutivity). Let $\mathrm{R}_{k} \in \mathrm{J}_{k} \pi$ be a partial differential equation. If there exists a quasi-regular basis for $\mathrm{T}_{\pi_{k}\left(p_{k}\right)}^{*} \mathrm{Q}$, the symbol $\mathrm{G}_{k}$ is involutive at $p_{k} \in \mathrm{R}_{k}$.

We now have the required machinery for the following central theorem for formal integrability [19].

TheOrem 3.20 (Goldschmidt). Let $(\mathrm{E}, \pi, \mathrm{Q})$ be a fibered manifold and $\mathrm{R}_{k} \subset \mathrm{J}_{k} \pi$ a partial differential equation. Assume the following hypotheses:

1. $\rho_{1}\left(\mathrm{R}_{k}\right)$ is a fibered submanifold of $\mathrm{J}_{k+1} \pi$;

2. $\hat{\pi}_{k}^{k+1}: \rho_{1}\left(\mathrm{R}_{k}\right) \rightarrow \mathrm{R}_{k}$ is an epimorphism of fibered manifolds;

3. $\mathrm{G}_{k}$ is 2-acyclic.

Then $\mathrm{R}_{k}$ is formally integrable.

Proof. [Sketch of the proof] Let $\Phi$ be the local morphism associated to $\mathrm{R}_{k}$ and recall the affine structure of $\rho_{1}\left(\mathrm{R}_{k}\right)$ over $\mathrm{R}_{k}$. Since $\rho_{1}\left(\mathrm{R}_{k}\right)$ is a fibered submanifold of $\mathrm{J}_{k+1} \pi$, we have $\mathrm{G}_{k+1}=\rho_{1}\left(\mathrm{G}_{k}\right)$ as a vector bundle over $\mathrm{R}_{k}$ and so one can define a vector bundle $\mathbf{C}=\operatorname{coker}\left(\rho_{1}(\sigma(\Phi))\right)$ such that the following sequence is exact:

$$
0 \longrightarrow \mathrm{G}_{k+1} \longrightarrow \mathrm{S}_{k+1} \mathrm{~T}^{*} \mathrm{Q} \otimes \mathrm{V} \pi \stackrel{\rho_{1}(\sigma(\Phi))}{\longrightarrow} \mathrm{T}^{*} \mathrm{Q} \otimes \mathrm{V} \pi^{\prime} \stackrel{\tau}{\longrightarrow} \mathrm{C} \longrightarrow 0
$$

where $\tau$ is the canonical projection onto $\mathrm{C}$. The essence of the proof is the construction of a map $\kappa: \mathrm{R}_{k} \rightarrow \mathrm{C}$ as follows: Consider the following exact and commutative 
diagram where the upper row is a sequence of vector bundles on which the second row of affine bundles are modeled:

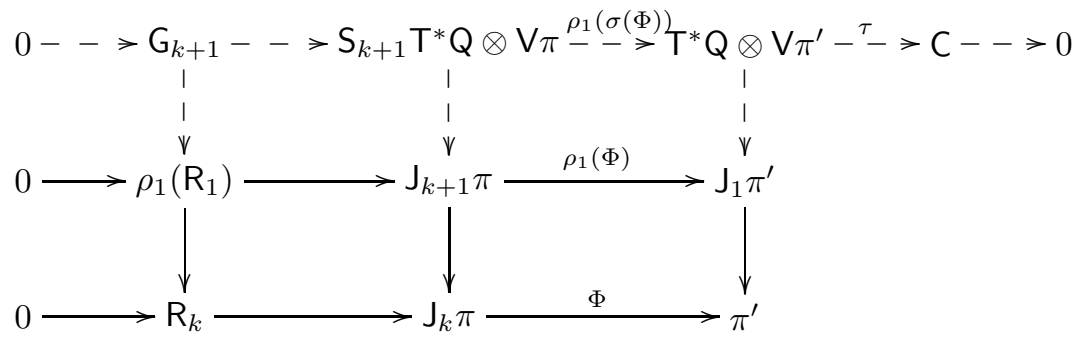

Let $p \in \mathrm{R}_{k}$ with $\pi_{k}(p)=q \in \mathrm{Q}$ and let $p^{\prime} \in \mathrm{J}_{k+1} \pi$ projecting to $p$. By commutativity of the diagram, $\rho_{1}(\Phi)\left(p^{\prime}\right)$ projects to $\Phi(p)$. As a result

$$
\rho_{1}(\Phi)\left(p^{\prime}\right)-j_{1} \Phi(p) \in \mathrm{T}^{*} \mathrm{Q} \otimes \mathrm{V} \pi^{\prime} .
$$

Let

$$
\kappa(p)=\tau\left(\rho_{1}(\Phi)\left(p^{\prime}\right)-j_{1} \Phi(p)\right) .
$$

One can show that this definition is independent of the choice of $p^{\prime}$ [18]. This map is called the curvature map. A diagram chase shows that the map $\kappa$ is zero with respect to the zero section of the vector bundle $\mathrm{C}$ if and only if the map $\pi_{k}^{k+1}$ is an epimorphism of affine bundles. Moreover 2-acyclicity implies that $\hat{\pi}_{k+r}^{k+l}$ is also an epimorphism of affine bundles.

If the symbol is involutive, condition 3 is automatically satisfied. We have the following definition.

Definition 3.21. A partial differential equation $\mathrm{R}_{k}$ is called involutive at a point $p$ if

1. its associated morphism is of constant rank,

2. there exists a quasi-regular basis at $p$ and

3. the map $\hat{\pi}_{k}^{k+1}$ is surjective and is of constant rank in a neighborhood of $p$.

EXAMPLE 3.22. Consider the partial differential equation

$$
\operatorname{grad}(f)=X,
$$

where $f: \mathbb{R}^{3} \rightarrow \mathbb{R}$ and $X$ is a vector field on $\mathbb{R}^{3}$.

One can easily check that the symbol of this partial differential equation is identity and hence involutive. Since

$$
\frac{\partial^{2} f}{\partial x^{j} \partial x^{i}}=\frac{\partial X^{i}}{\partial x^{j}}, \quad i, j \in\{1,2,3\}
$$

solutions for $f$ exist if

$$
\frac{\partial X^{j}}{\partial x^{i}}=\frac{\partial X^{i}}{\partial x^{j}}, \quad i, j \in\{1,2,3\}
$$


i.e.

$$
\operatorname{curl}(X)=0
$$

3.2. The space of connections. In this section we fix a fibered manifold $(\mathrm{E}, \pi, \mathrm{Q})$. As before, we denote by $\mathrm{V} \pi$ and $\mathrm{J}_{k}(\pi)$, the vertical bundle and the bundle of k-jets of a fibered manifold $\pi$ respectively [31]. We start by defining what we mean by a connection. It is not hard to show that this definition is equivalent to the usual construction of a connection as a splitting of the total space of a bundle on which the connection is defined [30], [10].

Definition 3.23. A connection on a fibered manifold $(\mathrm{E}, \pi, \mathrm{Q})$ is a section $\mathcal{S}: \mathrm{E} \rightarrow \mathrm{J}^{1}(\pi)$ of the bundle $\pi_{0}^{1}: \mathrm{J}^{1}(\pi) \rightarrow \mathrm{E}$.

In a natural coordinates $\left(q^{i}, u^{a}, u_{k}^{a}\right)$ for $\mathrm{J}^{1}(\pi)$, a connection has the form $\left(q^{i}, u^{a}\right) \mapsto$ $\left(q^{i}, u^{a}, \mathcal{S}_{k}^{a}\right)$ which defines the connection coefficients $\mathcal{S}_{k}^{a}$, where $a \in\{1, \cdots, m\}$ and $i, k \in\{1, \cdots, n\}$. One can define the covariant derivative associated to a connection as follows.

Definition 3.24. Let $\mathcal{S}: \mathrm{E} \rightarrow \mathrm{J}^{1}(\pi)$ be a connection on a fibered manifold $(\mathrm{E}, \pi, \mathrm{Q})$. If $\xi$ is a smooth local section of $\mathrm{E}$, then the $\mathcal{S}$-covariant differential of $\xi$ is the smooth local section $\nabla^{\mathcal{S}} \xi$ of $\mathrm{T}^{*} \mathrm{Q} \otimes \xi^{*} \mathrm{~V}(\pi)$ defined by

$$
\nabla^{\mathcal{S}} \xi(q)=j_{1} \xi(q)-\mathcal{S}(\xi(q))
$$

In natural coordinates we have

$$
\nabla^{\mathcal{S}} \xi=\left(\frac{\partial \xi^{a}}{\partial q^{i}}-\mathcal{S}_{i}^{a}\right) d x^{i} \otimes \frac{\partial}{\partial u^{a}}
$$

If $X$ is a vector field on $\mathrm{Q}$, then the $\mathcal{S}$-covariant derivative of $\xi$ with respect to $X$ is the section of $\xi^{*} \mathrm{~V}(\pi)$ defined by $\nabla_{X}^{\mathcal{S}} \xi=\nabla^{\mathcal{S}} \xi(X)$. A linear connection on a vector bundle $(\mathrm{E}, \pi, \mathrm{Q})$ is a connection $\mathcal{S}: \mathrm{E} \rightarrow \mathrm{J}^{1}(\pi)$ that is also a vector bundle morphism over $\mathrm{id}_{\mathrm{E}}$. In adapted coordinates $\left(x^{i}, u^{a}\right)$ on $\mathrm{E}$ and $\left(x^{i}, u^{a}, u_{k}^{a}\right)$ on $\mathrm{J}^{1}(\pi)$, a linear connection has the form $\left(x^{i}, u^{a}\right) \rightarrow\left(x^{i}, u^{a}, \mathcal{S}_{k b}^{a} u^{b}\right)$ which defines the connection coefficients $\mathcal{S}_{i b}^{a}$ where $a, b \in\{1, \cdots, m\}$ and $i, k \in\{1, \cdots, n\}$.

A linear connection $\mathcal{S}$ on the vector bundle $\left(\mathrm{TQ}, \pi_{\mathrm{Q}}, \mathrm{Q}\right)$ is sometimes called an affine connection on $\mathrm{Q}$. We have the following proposition which generalizes to vector bundles.

Proposition 3.25. The set of affine connections on a manifold $Q$ is the set of sections of an affine subbundle of the vector bundle $T^{*} \mathrm{Q} \otimes \mathrm{J}^{1}\left(\pi_{\mathrm{Q}}\right)$ over $\mathrm{Q}$ modeled on the vector bundle $\mathrm{T}^{*} \mathrm{Q} \otimes \mathrm{T}^{*} \mathrm{Q} \otimes \mathrm{TQ}$.

The following proposition clarifies the structure of the space of torsion-free affine connections.

Proposition 3.26. The set of torsion-free affine connections on a manifold $\mathrm{Q}$ is an affine subbundle of the vector bundle $\mathrm{T}^{*} \mathrm{Q} \otimes \mathrm{J}^{1}\left(\pi_{\mathrm{Q}}\right)$ over $\mathrm{Q}$ modeled on the vector 
bundle $\mathrm{S}_{2} \mathrm{~T}^{*} \mathrm{Q} \otimes \mathrm{TQ}$ given by

$\operatorname{Aff}_{0}(\mathrm{Q})=$

(6)

$\left\{\Xi \in \mathrm{T}^{*} \mathrm{Q} \otimes \mathrm{J}^{1}\left(\pi_{\mathrm{Q}}\right) \mid \pi_{0}^{1} \circ \Xi=\mathrm{id}_{\mathrm{TQ}},\left(j_{1} Y-\Xi(Y)\right)(X)-\left(j_{1} X-\Xi(X)\right)(Y)=[X, Y]\right\}$,

where $X, Y \in \Gamma^{\omega}(\mathrm{TQ})$ and we thought of $\Xi$ as a section $\mathrm{TQ} \rightarrow \mathrm{J}^{1}\left(\pi_{\mathrm{Q}}\right)$ for defining $\pi_{0}^{1} \circ \Xi$.

3.3. Ricci identity. Let $(E, \pi, Q)$ be a vector bundle. There is a bijective correspondence between the set of linear connections $\mathcal{S}: \mathrm{E} \rightarrow \mathrm{J}_{1} \pi$ and the type (1,1)-tensor fields $\mathcal{P}_{\mathcal{S}}^{H}$ on $\mathrm{E}$, where $\mathcal{P}_{\mathcal{S}}^{H}$ is a projection operator of constant rank, $\mathcal{P}_{\mathcal{S}}^{H}(X)=0$ for every $X \in \Gamma^{\omega}(\mathrm{V} \pi)$ and $\operatorname{Im}\left(\mathcal{P}_{\mathcal{S}}^{H}\right) \oplus \mathrm{V} \pi=\mathrm{TE}$. Such a projection is called the horizontal projection associated to the connection $\mathcal{S}$ [31]. An integral section of a connection $\mathcal{S}$ is an analytic local section $\xi$ of $\pi$ satisfying $j_{1} \xi=\mathcal{S}(\xi)$. There is no guarantee that such a section exists even locally. The existence of such an integral section is equivalent to the vanishing of the Nijenhuis tensor of the $(1,1)$-tensor field $\mathcal{P}_{\mathcal{S}}^{H}$ ( see $[31,23]$ ). In other words, the Nijenhuis tensor measures the involutivity of the associated horizontal subbundle, and as a result, the Nijenhuis tensor of $\mathcal{S}$ is directly related to the curvature tensor $\mathrm{R}[\mathcal{S}]$ associated to $\mathcal{S}$. Let $\left(q^{i}, u^{a}\right)$ be an adapted local coordinates on a neighborhood $U$ of $\mathrm{E}$ with $i \in\{1, \cdots, n\}$ and $a \in\{1, \cdots, m\}$. Also let $\left\{e_{a}\right\}$ be a basis for the local sections of $E$. The curvature tensor, $R[\mathcal{S}] \in \Gamma^{\omega}\left(E^{*} \otimes E \otimes \Lambda_{2} T^{*} Q\right)$, can be written as

$$
\mathrm{R}[\mathcal{S}]_{i j b}^{a}=\frac{\partial \mathcal{S}_{i b}^{a}}{\partial x^{j}}+\mathcal{S}_{i c}^{a} \mathcal{S}_{j b}^{c}-\left(\frac{\partial \mathcal{S}_{j b}^{a}}{\partial x^{i}}+\mathcal{S}_{j c}^{a} \mathcal{S}_{i b}^{c}\right)
$$

where $i, j \in\{1, \cdots, n\}$ and $a, b, c \in\{1, \cdots, m\}$. One can naturally define an induced connection on the fibered product as follows. Let $\left(E_{1}, \pi_{1}, Q\right)$ and $\left(E_{2}, \pi_{2}, Q\right)$ be two vector bundles equipped with two connections $\mathcal{S}_{1}$ and $\mathcal{S}_{2}$, respectively. There is a unique connection $\mathcal{S}_{1} \otimes_{\mathrm{Q}} \mathcal{S}_{2}$ that makes the following diagram commute:

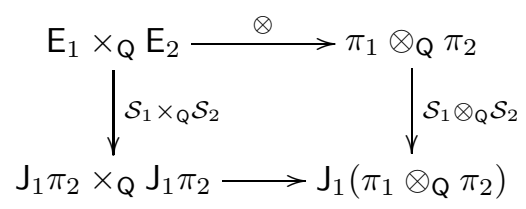

For more information about the induced connection on a fibered product bundle see [31]. One can use the same procedure to induce a connection $\mathcal{S}^{*}$ on the dual bundle $\pi^{*}$. For our purposes, we consider the tensor bundle $\left(\mathrm{E} \otimes_{\mathrm{Q}} \mathrm{E}, \pi \otimes_{\mathrm{Q}} \pi, \mathrm{Q}\right)$ where $\pi$ is a vector bundle. Let $\left(x^{i}\right)$ be local coordinates for $\mathrm{Q}$ and let $\left(x^{i}, u^{a}\right)$ be adapted coordinates for $\mathrm{E}$, where $i \in\{1, \cdots, n\}$ and $a \in\{1, \cdots, m\}$. Denote an analytic local section of $\mathrm{E} \otimes \mathrm{E}$ by $\xi=\xi^{a b} e_{a} \otimes e_{b}$ where $\left\{e_{a}\right\}$ is a basis for local sections of $\mathrm{E}$ 
and $a, b \in\{1, \cdots, m\}$. Then the covariant derivative with respect to $\mathcal{S} \otimes \mathcal{S}$ can be represented by

$$
\nabla^{\mathcal{S} \otimes \mathcal{S}} \xi=j_{1} \xi-(\mathcal{S} \otimes \mathcal{S})(\xi)
$$

We have the following representation of the covariant derivative with respect to the induced connection $\mathcal{S} \otimes \mathcal{S}$ in local coordinates:

$$
\left(\nabla^{\mathcal{S} \otimes \mathcal{S}} \xi\right)_{i}^{a b}=\frac{\partial \xi^{a b}}{\partial x^{i}}-\mathcal{S}_{i c}^{a} \xi^{c b}-\mathcal{S}_{i c}^{b} \xi^{a c},
$$

where $c \in\{1, \cdots, m\}$ and $a, b, c \in\{1, \cdots, m\}$. Using equation (7), one can show that the associated curvature tensor for $\mathcal{S} \otimes \mathcal{S}$ is

$$
\mathrm{R}[\mathcal{S} \otimes \mathcal{S}]_{c d i j}^{a b} \xi^{c d}=\mathrm{R}[\mathcal{S}]_{c i j}^{a} \xi^{c b}+\mathrm{R}[\mathcal{S}]_{c i j}^{b} \xi^{a c}
$$

The vanishing of the curvature tensor is an obstruction for the involutivity of the horizontal subspace of $\mathrm{T}(\mathrm{E} \otimes \mathrm{E})$ associated with the induced connection $\mathcal{S} \otimes \mathcal{S}$. The relation between the curvature tensor of a product bundle and the curvature of the underlying bundles leads to the Ricci identity, [12]. In the literature this identity is typically introduced through the following lemma:

Lemma 3.27. Let $(\mathrm{Q}, \mathbb{G})$ be a Riemannian manifold equipped with a symmetric affine connection $\mathcal{S}$. Then the following identity holds and is called the Ricci identity:

$$
\left(\nabla_{X} \nabla_{Y} \mathbb{G}-\nabla_{Y} \nabla_{X} \mathbb{G}-\nabla_{[X, Y]} \mathbb{G}\right)(Z, W)=\mathbb{G}(R(X, Y) Z, W)+\mathbb{G}(Z, R(X, Y) W) .
$$

where $X, Y, Z, W \in \Gamma^{\omega}(\mathrm{TQ})$

Proof. The proof follows from a direct computation using equation (8) to compute the covariant derivative of $\mathbb{G}$ with respect to a vector field.

REMARK 3.28. We state the following remarks for future use.

1. Lemma 3.27 can be extended to any $(0,2)$-tensor on $\mathrm{Q}$, but for our purposes we state the lemma for $\mathbb{G} \in \mathrm{S}_{2}^{+}\left(\mathrm{T}^{*} \mathrm{Q}\right)$.

2. The Ricci identity appears when one tries to find a set of necessary conditions for a metric to be associated to a given symmetric affine connection; see [16], [36].

4. Geometric formulation of partial differential equations in energy shaping. We give a formulation of the partial differential equations of the energy shaping problem using the theory of partial differential equations presented in the previous section. This formulation is an integral part of our approach since it places the energy shaping problem separately into the realm of the formal theory of partial differential equations.

4.1. Kinetic energy shaping. We provide a jet bundle structure associated to the kinetic energy shaping system of partial differential equations. This system of 
partial differential equations involves the affine subbundle description given for the set of torsion free connection; see Section 3.2.

Let $\left(\mathrm{S}_{2}^{+} \mathrm{T}^{*} \mathrm{Q}, \pi_{\mathcal{G}}, \mathrm{Q}\right)$ be the bundle of Riemannian metrics on the configuration manifold $Q$. One can generalize the definitions in this section by allowing metrics with other signatures; see Remark 4.7. Let $\left(\mathrm{B}, \pi_{\mathrm{B}}, \mathrm{Q}\right)$ be the bundle of gyroscopic tensor fields over $Q$ where $B \doteq \operatorname{Gyr}(T Q) \cap \operatorname{ker}($ Alt $)$. We have the following definition.

DeFINITION 4.1. The kinetic energy shaping bundle is the fibered product bundle $\left(K S, \pi \doteq \pi_{\mathcal{G}} \times_{\mathrm{Q}} \pi_{\mathrm{B}}, \mathrm{Q}\right)$, where $K S \doteq \mathrm{S}_{2}^{+} \mathrm{T}^{*} \mathrm{Q} \times{ }_{\mathrm{Q}} \mathrm{B}$. We denote by $\pi_{1}$ and $\pi_{2}$ the projection on the first and second factors.

In local coordinates, a typical fiber over $q \in \mathrm{Q}$ is a pair $(\mathbb{G}(q), \mathbb{B}(q))$ and a typical point of $\mathrm{J}^{1}(\pi)$ is given by $\left(q^{i}, \mathbb{G}_{m n}, \mathbb{B}_{l p q}, \mathbb{G}_{j k, a}, \mathbb{B}_{l p q, b}\right)$ where we denote the derivatives of $\mathbb{G}_{j k}$ and $\mathbb{B}_{l p q}$, respectively, by $\mathbb{G}_{j k, a}$ and $\mathbb{B}_{l p q, b}$.

Define the "Levi-Civita" map $\phi_{L C}: J_{1} \pi_{\mathcal{G}} \rightarrow \operatorname{Aff}_{0}(\mathrm{Q})$ by $\phi_{L C}\left(j_{1} \mathbb{G}\right)=\nabla^{\mathbb{G}}$. Let $\Sigma_{\mathrm{ol}}=\left(\mathrm{Q}, \mathbb{G}_{\mathrm{ol}}, V_{\mathrm{ol}}, 0, \mathcal{W}_{\mathrm{ol}}\right)$ be a given open-loop simple mechanical control system and let $(\mathrm{KS}, \pi, \mathrm{Q})$ be the kinetic energy shaping bundle. The point $p_{0}=\left(q_{0}, \mathbb{G}_{\mathrm{ol}}, 0\right) \in \mathrm{KS}$, where $q_{0} \in \mathrm{Q}$, represents the open-loop simple mechanical control system $\Sigma_{\mathrm{ol}}$. We define the following projection:

$$
\pi_{\mathcal{W}}: \mathbb{G}_{\mathrm{ol}}^{\sharp}\left(\mathrm{T}^{*} \mathrm{Q} \otimes \mathrm{S}_{2}^{+} \mathrm{T}^{*} \mathrm{Q}\right) \rightarrow \mathbb{G}_{\mathrm{ol}}^{\sharp}\left(\mathrm{T}^{*} \mathrm{Q} \otimes \mathrm{S}_{2}^{+} \mathrm{T}^{*} \mathrm{Q}\right) / \mathbb{G}_{\mathrm{ol}}^{\sharp}\left(\mathcal{W}_{\mathrm{ol}} \otimes \mathrm{S}_{2}^{+} \mathrm{T}^{*} \mathrm{Q}\right) \doteq \mathcal{K},
$$

where we used the extended definition of sharp map; see equation (1). We now have the required tools for defining the kinetic energy shaping partial differential equation as a submanifold of $J_{1} \pi$.

DeFINITION 4.2. Let (KS, $\pi, \mathrm{Q})$ be the kinetic energy shaping bundle and let $p_{0}=$ $\left(q_{0}, \mathbb{G}_{\mathrm{ol}}, 0\right) \in \mathrm{KS}$ where $q_{0} \in \mathrm{Q}$. If $\pi_{\mathcal{W}}$ and $\phi_{L C}$ are, respectively, the projection and the affine connection map defined above, the kinetic energy shaping submanifold $\mathrm{R}_{\text {kin }}\left(p_{0}\right) \subset \mathrm{J}_{1} \pi$ is defined by

$$
\mathrm{R}_{\mathrm{kin}}\left(p_{0}\right)=\left\{p \in \mathrm{J}_{1} \pi \quad \mid \Phi_{\text {kin }}(p)=0\right\},
$$

where $\Phi_{\text {kin }}$ is the kinetic energy shaping map given by

$$
\Phi_{\text {kin }}(p)=\pi_{\mathcal{W}}\left(\phi_{L C}\left(j_{1} \pi_{1}(p)\right)-\phi_{L C}\left(j_{1} \pi_{1}\left(p_{0}\right)\right)\right)-\pi_{\mathcal{W}}\left(\pi_{1}(p)\right)^{\sharp} \pi_{2}(p) .
$$

One can represent the governing system of partial differential equations for the kinetic energy shaping problem by the following exact sequence:

$$
0 \longrightarrow \mathrm{R}_{\mathrm{kin}}\left(p_{0}\right) \longrightarrow \mathrm{J}_{1} \pi \stackrel{\Phi_{\mathrm{kin}}}{\longrightarrow} \mathcal{K},
$$

where $R_{\text {kin }}$ is the kernel of $\Phi_{\text {kin }}$ with respect to the zero section of $\mathcal{K}$.

4.2. The $\lambda$-method. In this section, we recall a differential geometric approach to the kinetic energy shaping problem from $[5,3]$. The main idea is to transform the 
set of quasi-linear partial differential equations from the previous section into a set of linear partial differential equations in terms of a new variable. In the following definition we introduce a set of partial differential equations which is the main component of this equivalent system.

The following theorem gives the desired transformation.

TheOREM 4.3. Let $\Sigma_{\mathrm{ol}}=\left(\mathrm{Q}, \mathbb{G}_{\mathrm{ol}}, V_{\mathrm{ol}}, \mathcal{F}_{\mathrm{ol}}, \mathcal{W}_{\mathrm{ol}}\right)$ be an open-loop simple mechanical control system. Let $P \in \Gamma^{\omega}\left(\mathrm{T}^{*} \mathrm{Q} \otimes \mathrm{TQ}\right)$ be the $\mathbb{G}_{\mathrm{ol}}$-orthogonal projection as above. Let $\mathbb{G}_{\mathrm{cl}} \in \Gamma^{\omega}\left(\mathrm{S}_{2}^{+} \mathrm{T}^{*} \mathrm{Q}\right)$ and let $\mathbb{B}$ be a quadratic gyroscopic tensor. If $\mathbb{G}_{\mathrm{ol}}^{b}=\mathbb{G}_{\mathrm{cl}}^{b} \circ \lambda$ for $\lambda \in \Gamma^{\omega}\left(\mathrm{T}^{*} \mathrm{Q} \otimes \mathrm{TQ}\right)$, the following two conditions are equivalent:

1. $P\left(\nabla_{X}^{\mathbb{G}_{c l}} X-\nabla_{X}^{\mathbb{G}_{\mathrm{ol}}} X-\mathbb{G}_{\mathrm{cl}}^{\sharp} \circ \mathbb{B}^{\mathrm{b}}(X)\right)=0, \quad \forall X \in \Gamma^{\omega}(\mathrm{TQ})$;

2. (a) $\nabla_{Z}^{\mathbb{G}_{\text {ol }}}\left(\mathbb{G}_{\mathrm{ol}} \lambda\right)(P X, P Y)+\frac{1}{2}\langle\mathbb{B}(\lambda P X, \lambda P Y)+\mathbb{B}(\lambda P Y, \lambda P X), Z\rangle=0$, and

(b) $\nabla_{\lambda P X}^{\mathbb{G}_{\mathrm{ol}}} \mathbb{G}_{\mathrm{cl}}(Z, Z)+2 \mathbb{G}_{\mathrm{cl}}\left(\nabla_{Z}^{\mathbb{G}_{\mathrm{ol}}} \lambda P X, Z\right)=2 \mathbb{G}_{\mathrm{ol}}\left(\nabla_{Z}^{\mathbb{G}_{\mathrm{ol}}} P X, Z\right)$

$-2\left\langle\lambda P X, \mathbb{B}^{b}(Z)\right\rangle$,

where $X, Y, Z \in \Gamma^{\omega}(\mathrm{TQ})$.

In order to prove this theorem we need the following lemma.

Lemma 4.4. Let $(\mathrm{Q}, \mathbb{G})$ be a Riemannian manifold and let $\mathcal{W}$ be a codistribution on $\mathrm{Q}$. Let $P \in \Gamma^{\omega}\left(\mathrm{T}^{*} \mathrm{Q} \otimes \mathrm{TQ}\right)$ be the $\mathbb{G}$-orthogonal projection introduced above and let $\mathbb{G}_{\mathrm{cl}} \in \Gamma^{\omega}\left(\mathrm{S}_{2}^{+} \mathrm{T}^{*} \mathrm{Q}\right)$. If

$$
P\left(\nabla_{X}^{\mathbb{G}_{c l}} X-\nabla_{X}^{\mathbb{G}} X-\mathbb{G}_{\mathrm{cl}}^{\sharp} \circ \mathbb{B}^{b}(X)\right)=0, \quad \forall X \in \Gamma^{\omega}(\mathrm{TQ}),
$$

then

1. for $X, Y \in \Gamma^{\omega}(\mathrm{TQ})$ we have

$$
P\left(\nabla_{X}^{\mathbb{G}} Y-\nabla_{X}^{\mathbb{G}_{c l}} Y\right)=-\frac{1}{2} P \mathbb{G}_{\mathrm{cl}}^{\sharp} \circ\left(\mathbb{B}^{b}(X+Y)-\mathbb{B}^{b}(X)-\mathbb{B}^{b}(Y)\right),
$$

and

2. for $\mathbb{G}=\mathbb{G}_{\mathrm{cl}} \circ \lambda$ for $\lambda \in \Gamma^{\omega}\left(\mathrm{T}^{*} \mathrm{Q} \otimes \mathrm{TQ}\right)$ we have

$$
2 \mathbb{G}\left(P\left(\nabla_{\lambda P X}^{\mathbb{G}} Z-\nabla_{\lambda P X}^{\mathbb{G}_{c l}} Z\right), X\right)=\nabla_{Z}^{\mathbb{G}}(\mathbb{G} \lambda)(P X, P X),
$$

where $X, Z \in \Gamma^{\omega}(\mathrm{TQ})$.

Proof. We begin with the first statement. Note that since the connections are torsion free,

$$
\nabla_{X}^{\mathbb{G}} Y-\nabla_{X}^{\mathbb{G}_{c l}} Y=\nabla_{Y}^{\mathbb{G}} X-\nabla_{Y}^{\mathbb{G}_{c l}} X
$$

We have

$$
\begin{aligned}
P\left(\nabla_{X}^{\mathbb{G}} Y-\nabla_{X}^{\mathbb{G}_{c l}} Y\right)= & \frac{1}{2} P\left(\nabla_{X}^{\mathbb{G}} Y-\nabla_{X}^{\mathbb{G}_{c l}} Y+\nabla_{Y}^{\mathbb{G}} X-\nabla_{Y}^{\mathbb{G}_{c l}} X\right) \\
= & \frac{1}{2} P\left(\nabla_{X+Y}^{\mathbb{G}}(X+Y)-\nabla_{X+Y}^{\mathbb{G}_{c l}}(X+Y)\right) \\
& -\frac{1}{2} P\left(\nabla_{X}^{\mathbb{G}}(X)-\nabla_{X}^{\mathbb{G}_{c l}}(X)\right)-\frac{1}{2} P\left(\nabla_{Y}^{\mathbb{G}}(Y)-\nabla_{Y}^{\mathbb{G}_{c l}}(Y)\right) \\
= & -\frac{1}{2} P \mathbb{G}_{\mathrm{cl}}^{\sharp} \circ\left(\mathbb{B}^{b}(X+Y)-\mathbb{B}^{b}(X)-\mathbb{B}^{b}(Y)\right),
\end{aligned}
$$


where $X, Y \in \Gamma^{\omega}(\mathrm{TQ})$.

For the second part, recall that for the Levi-Civita connection $\nabla^{\mathbb{G}}$ associated to $\mathbb{G}$ one can write

$$
\begin{aligned}
2 \mathbb{G}\left(\nabla_{X}^{\mathbb{G}} Y, Z\right)= & L_{X} \mathbb{G}(Y, Z)+L_{Y} \mathbb{G}(Z, X)-L_{Z} \mathbb{G}(X, Y) \\
& +\mathbb{G}([X, Y], Z)+\mathbb{G}([Z, X], Y)-\mathbb{G}([Y, Z], X) .
\end{aligned}
$$

Moreover, we have

$$
L_{X}(\mathbb{G}(Y, Z))=\mathbb{G}\left(\nabla_{X}^{\mathbb{G}} Y, Z\right)+\mathbb{G}\left(Y, \nabla_{X}^{\mathbb{G}} Z\right) .
$$

Thus

$$
\begin{aligned}
2 \mathbb{G}\left(P\left(\nabla_{\lambda P X}^{\mathbb{G}} Z-\nabla_{\lambda P X}^{\mathbb{G}_{\mathrm{cl}}} Z\right), X\right) & =2 \mathbb{G}\left(\nabla_{\lambda P X}^{\mathbb{G}} Z, P X\right)-2 \mathbb{G}\left(\nabla_{\lambda P X}^{\mathbb{G}_{\mathrm{cl}}} Z, P X\right) \\
& =2 \mathbb{G}\left(\nabla_{\lambda P X}^{\mathbb{G}} Z, P X\right)-2 \mathbb{G}_{\mathrm{cl}}\left(\nabla_{\lambda P X}^{\mathbb{G}_{\mathrm{cl}}} Z, \lambda P X\right) .
\end{aligned}
$$

We use equation (13) to get

$$
\begin{aligned}
2 \mathbb{G}\left(P \left(\nabla_{\lambda P X}^{\mathbb{G}} Z-\right.\right. & \left.\left.\nabla_{\lambda P X}^{\mathbb{G}_{c 1}} Z\right), X\right)=\lambda P X \mathbb{G}(Z, P X)-P X \mathbb{G}(\lambda P X, Z) \\
& +\mathbb{G}([P X, \lambda P X], Z)-\mathbb{G}([Z, P X], \lambda P X)+\mathbb{G}([Z, \lambda P X], P X) .
\end{aligned}
$$

Expanding the terms along with equation (14), and after some simplifications, we have

$$
2 \mathbb{G}\left(P\left(\nabla_{\lambda P X}^{\mathbb{G}} Z-\nabla_{\lambda P X}^{\mathbb{G}_{\mathrm{cl}}} Z\right), X\right)=\mathbb{G}\left(\nabla_{Z}^{\mathbb{G}}(\lambda P X), P X\right)-\mathbb{G}\left(\nabla_{Z}^{\mathbb{G}} P X, \lambda P X\right) .
$$

Note that

$$
L_{Z}(\lambda(X))=\nabla_{Z}^{\mathbb{G}}(\lambda)(X)+\lambda\left(\nabla_{Z}^{\mathbb{G}} X\right) .
$$

As a result,

$$
\begin{aligned}
2 \mathbb{G}\left(P\left(\nabla_{\lambda P X}^{\mathbb{G}} Z-\nabla_{\lambda P X}^{\mathbb{G}_{c l}} Z\right), X\right)= & \mathbb{G}\left(\left(\nabla_{Z}^{\mathbb{G}} \lambda\right)(P X)+\lambda\left(\nabla_{Z}^{\mathbb{G}} P X\right), P X\right) \\
& -\mathbb{G}\left(\nabla_{Z}^{\mathbb{G}} P X, \lambda P X\right) \\
= & \mathbb{G}\left(\left(\nabla_{Z}^{\mathbb{G}} \lambda\right)(P X), P X\right)+\mathbb{G}\left(\lambda\left(\nabla_{Z}^{\mathbb{G}} P X\right), P X\right) \\
& -\mathbb{G}\left(\nabla_{Z}^{\mathbb{G}} P X, \lambda P X\right) \\
= & \mathbb{G}\left(\left(\nabla_{Z}^{\mathbb{G}} \lambda\right) P X, P X\right) .
\end{aligned}
$$

Since $\nabla^{\mathbb{G}} \mathbb{G}=0$, one can write

$$
2 \mathbb{G}\left(P\left(\nabla_{\lambda P X}^{\mathbb{G}} Z-\nabla_{\lambda P X}^{\mathbb{G}_{c l}} Z\right), X\right)=\nabla_{Z}^{\mathbb{G}}(\mathbb{G} \lambda)(P X, P X),
$$

which is the desired result.

Proof. [Proof of Theorem $4.3(1) \Rightarrow(2)$ ] We first assume that $\mathbb{G}_{\mathrm{cl}}$ and $\mathbb{B}$ satisfy the kinetic energy shaping equation

$$
P\left(\nabla_{X}^{\mathbb{G}_{\mathrm{cl}}} X-\nabla_{X}^{\mathbb{G}_{\text {ol }}} X-\mathbb{G}_{\mathrm{cl}}^{\sharp} \circ \mathbb{B}^{b}(X)\right)=0, \quad \forall X \in \Gamma^{\omega}(\mathrm{TQ}) .
$$


Using the second part of lemma 4.4 we have

$$
2 \mathbb{G}_{\mathrm{ol}}\left(P\left(\nabla_{\lambda P X}^{\mathbb{G}_{\mathrm{ol}}} Z-\nabla_{\lambda P X}^{\mathbb{G}_{\mathrm{cl}}} Z\right), X\right)=\nabla_{Z}\left(\mathbb{G}_{\mathrm{ol}} \lambda\right)(P X, P X) .
$$

By the first part of lemma 4.4 we have

$$
P\left(\nabla_{\lambda P X}^{\mathbb{G}_{\mathrm{ol}}} Z-\nabla_{\lambda P X}^{\mathbb{G}_{\mathrm{cl}}} Z\right)=-\frac{1}{2} P \mathbb{G}_{\mathrm{cl}}^{\sharp} \circ\left(\mathbb{B}^{\mathrm{b}}(\lambda P X+Z)-\mathbb{B}^{\mathrm{b}}(\lambda P X)-\mathbb{B}^{\mathrm{b}}(Z)\right) .
$$

Substituting equation (16) we get

$$
2 \mathbb{G}_{\mathrm{ol}}\left(-\frac{1}{2} P \mathbb{G}_{\mathrm{cl}}^{\sharp} \circ\left(\mathbb{B}^{b}(\lambda P X+Z)-\mathbb{B}^{b}(\lambda P X)-\mathbb{B}^{b}(Z)\right), X\right)=\nabla_{Z}\left(\mathbb{G}_{\mathrm{ol}} \lambda\right)(P X, P X),
$$

which can be written as

$$
\nabla_{Z}\left(\mathbb{G}_{\mathrm{ol}} \lambda\right)(P X, P X)+\left\langle\mathbb{B}^{\mathrm{b}}(\lambda P X+Z)-\mathbb{B}^{\mathrm{b}}(\lambda P X)-\mathbb{B}^{\mathrm{b}}(Z), \lambda P X\right\rangle=0 .
$$

From the definition of the flat operation we have $\left\langle\mathbb{B}^{\mathrm{b}}(Y), X\right\rangle=\mathbb{B}(X, Y, Y)$. Also recall that the gyroscopic tensor is antisymmetric in the first two elements. We can then expand the right hand side of the previous equation to get

$$
\nabla_{Z}\left(\mathbb{G}_{\mathrm{ol}} \lambda\right)(P X, P X)=\mathbb{B}(\lambda P X, Z, \lambda P X)=-\langle\mathbb{B}(\lambda P X, \lambda P X), Z\rangle .
$$

Notice that $\mathbb{G}_{\mathrm{ol}} \lambda$ and $\nabla_{Z}\left(\mathbb{G}_{\mathrm{ol}} \lambda\right)$ are both symmetric $(0,2)$ tensors. Thus we have

$$
\nabla_{Z}\left(\mathbb{G}_{\mathrm{ol}} \lambda\right)(P X, P Y)+\frac{1}{2}\langle\mathbb{B}(\lambda P X, \lambda P Y)+\mathbb{B}(\lambda P Y, \lambda P X), Z\rangle=0,
$$

for all $X, Y \in \Gamma^{\omega}(\mathrm{TQ})$ as claimed. Now, for the second part of the proof, we have

$$
\begin{aligned}
L_{\lambda P X} \mathbb{G}_{\mathrm{cl}}(Z, Z) & =\mathbb{G}_{\mathrm{cl}}\left(\nabla_{\lambda P X}^{\mathbb{G}_{c l}} Z, Z\right)+\mathbb{G}_{\mathrm{cl}}\left(Z, \nabla_{\lambda P X}^{\mathbb{G}_{c l}} Z\right) \\
& =2 \mathbb{G}_{\mathrm{cl}}\left(\nabla_{\lambda P X}^{\mathbb{G}_{c l}} Z, Z\right) \\
& =2 \mathbb{G}_{\mathrm{cl}}\left(\nabla_{Z}^{\mathbb{G}_{\mathrm{cl}}} \lambda P X-[Z, \lambda P X], Z\right) \\
& =2 \mathbb{G}_{\mathrm{cl}}\left(\nabla_{Z}^{\mathbb{G}_{\mathrm{cl}}} \lambda P X, Z\right)-2 \mathbb{G}_{\mathrm{cl}}([Z, \lambda P X], Z) .
\end{aligned}
$$

As a result

$$
\begin{aligned}
L_{\lambda P X} \mathbb{G}_{\mathrm{cl}}(Z, Z)+2 \mathbb{G}_{\mathrm{cl}}([Z, \lambda P X], Z) & =2 \mathbb{G}_{\mathrm{cl}}\left(\nabla_{Z}^{\mathbb{G}_{\mathrm{cl}}} \lambda P X, Z\right) \\
& =2 L_{Z} \mathbb{G}_{\mathrm{ol}}(P X, Z)-2 \mathbb{G}_{\mathrm{ol}}\left(P X, \nabla_{Z}^{\mathbb{G}_{\mathrm{cl}}} Z\right) \\
& =2 L_{Z} \mathbb{G}_{\mathrm{ol}}(P X, Z)-2 \mathbb{G}_{\mathrm{ol}}\left(X, P \nabla_{Z}^{\mathbb{G}_{\mathrm{cl}}} Z\right) .
\end{aligned}
$$

Now, from the kinetic energy shaping system of partial differential equations, we have

$$
P \nabla_{Z}^{\mathbb{G}_{\mathrm{cl}}} Z=P \nabla_{Z}^{\mathbb{G}_{\mathrm{ol}}} Z+P \mathbb{G}_{\mathrm{cl}}^{\sharp} \mathbb{B}^{b}(Z) .
$$

Therefore,

$$
\begin{aligned}
& L_{\lambda P X} \mathbb{G}_{\mathrm{cl}}(Z, Z)+2 \mathbb{G}_{\mathrm{cl}}([Z, \lambda P X], Z) \\
& =2 L_{Z} \mathbb{G}_{\mathrm{ol}}(P X, Z)-2 \mathbb{G}_{\mathrm{ol}}\left(X, P \nabla_{Z}^{\mathbb{G}_{\mathrm{ol}}} Z+P \mathbb{G}_{\mathrm{cl}}^{\sharp} \mathbb{B}^{\mathrm{b}}(Z)\right) \\
& =2 \mathbb{G}_{\mathrm{ol}}\left(\nabla_{Z}^{\mathbb{G}_{\mathrm{ol}}} P X, Z\right)+2 \mathbb{G}_{\mathrm{ol}}\left(P X, \nabla_{Z}^{\mathbb{G}_{\mathrm{ol}}} Z\right)-2 \mathbb{G}_{\mathrm{ol}}\left(X, P \nabla_{Z}^{\mathbb{G}_{\mathrm{ol}}} Z+P \mathbb{G}_{\mathrm{cl}}^{\sharp} \mathbb{B}^{\mathrm{b}}(Z)\right) .
\end{aligned}
$$


This gives

$$
L_{\lambda P X} \mathbb{G}_{\mathrm{cl}}(Z, Z)+2 \mathbb{G}_{\mathrm{cl}}([Z, \lambda P X], Z)=2 \mathbb{G}_{\mathrm{ol}}\left(\nabla_{Z}^{\mathbb{G}_{\mathrm{ol}}} P X, Z\right)-2\left\langle\lambda P X, \mathbb{B}^{b}(Z)\right\rangle .
$$

A simple computation gives the desired conclusion.

$(2) \Rightarrow(1)$ : We have to prove that if $\lambda=\mathbb{G}_{\mathrm{cl}}^{\sharp} \circ \mathbb{G}_{\mathrm{ol}}^{b}$ and if $\mathbb{G}_{\mathrm{cl}}$ and $\mathbb{B}$ satisfy the set of extended $\lambda$ equations and the closed-loop metric equation given in part 2 of the theorem, then $\left(\mathbb{G}_{\mathrm{cl}}, \mathbb{B}\right)$ is a solution to the kinetic energy shaping problem. We compute

$$
\begin{aligned}
& \mathbb{G}_{\mathrm{ol}}\left(P\left(\nabla_{X}^{\mathbb{G}_{\mathrm{ol}}} X-\nabla_{X}^{\mathbb{G}_{\mathrm{cl}}} X+\mathbb{G}_{\mathrm{cl}}^{\sharp} \mathbb{B}^{\mathrm{b}}(X)\right), Z\right) \\
&=\mathbb{G}_{\mathrm{ol}}\left(\nabla_{X}^{\mathbb{G}_{\mathrm{ol}}} X-\nabla_{X}^{\mathbb{G}_{\mathrm{cl}}} X+\mathbb{G}_{\mathrm{cl}}^{\sharp} \mathbb{B}^{b}(X), P Z\right) \\
&=\mathbb{G}_{\mathrm{ol}}\left(\nabla_{X}^{\mathbb{G}_{\mathrm{ol}}} X, P Z\right)-\mathbb{G}_{\mathrm{cl}}\left(\nabla_{X}^{\mathbb{G}_{\mathrm{cl}}} X, \lambda P Z\right)+\left\langle\mathbb{B}^{b}(X), \lambda P Z\right\rangle \\
&=L_{X} \mathbb{G}_{\mathrm{ol}}(X, P Z)-\mathbb{G}_{\mathrm{ol}}\left(X, \nabla_{X}^{\mathbb{G}_{\mathrm{ol}}} P Z\right) \\
& \quad-L_{X} \mathbb{G}_{\mathrm{cl}}(X, \lambda P Z)+\mathbb{G}_{\mathrm{cl}}\left(X, \nabla_{X}^{\mathbb{G}_{\mathrm{cl}}} \lambda P Z\right)+\left\langle\mathbb{B}^{b}(X), \lambda P Z\right\rangle \\
&=-\mathbb{G}_{\mathrm{ol}}\left(X, \nabla_{X}^{\mathbb{G}_{\mathrm{ol}}} P Z\right)+\mathbb{G}_{\mathrm{cl}}\left(X, \nabla_{X}^{\mathbb{G}_{\mathrm{cl}}} \lambda P Z\right)+\left\langle\mathbb{B}^{b}(X), \lambda P Z\right\rangle \\
&=-\mathbb{G}_{\mathrm{ol}}\left(X, \nabla_{X}^{\mathbb{G}_{\mathrm{ol}}} P Z\right)+\mathbb{G}_{\mathrm{cl}}\left(X, \nabla_{\lambda P}^{\mathbb{G}_{\mathrm{cl}}} X\right)+\mathbb{G}_{\mathrm{cl}}(X,[X, \lambda P Z])+\left\langle\mathbb{B}^{b}(X), \lambda P Z\right\rangle \\
&=--\mathbb{G}_{\mathrm{ol}}\left(X, \nabla_{X}^{\mathbb{G}_{\mathrm{ol}}} P Z\right)+\frac{1}{2} L_{\lambda P Z} \mathbb{G}_{\mathrm{cl}}(X, X)+\mathbb{G}_{\mathrm{cl}}(X,[X, \lambda P Z])+\left\langle\mathbb{B}^{\mathrm{b}}(X), \lambda P Z\right\rangle \\
&=-\mathbb{G}_{\mathrm{ol}}\left(X, \nabla_{X}^{\mathbb{G}_{\mathrm{ol}}} P Z\right)+\mathbb{G}_{\mathrm{ol}}\left(\nabla_{X}^{\mathbb{G}_{\mathrm{ol}}} P Z, X\right)-\left\langle\lambda P Z, \mathbb{B}^{b}(X)\right\rangle+\left\langle\mathbb{B}^{b}(X), \lambda P Z\right\rangle \\
&= 0,
\end{aligned}
$$

As a result,

$$
P\left(\nabla_{X}^{\mathbb{G}_{\text {ol }}} X-\nabla_{X}^{\mathbb{G}_{c l}} X+\mathbb{G}_{\mathrm{cl}}^{\sharp} \mathbb{B}^{b}(X)\right)=0,
$$

as desired.

From part 2 of the previous theorem we see that the kinetic energy shaping partial differential equation is equivalent to two partial differential equations, one for $\lambda$ and one for obtaining $\mathbb{G}_{\mathrm{cl}}$ from $\lambda$. We will study these partial differential equations in detail later in the paper, but for now let us define them.

Definition 4.5. Let $\mathrm{Q}$ be an n-dimensional manifold and let $\mathbb{G} \in \Gamma^{\omega}\left(\mathrm{S}_{2}^{+} \mathrm{T}^{*} \mathrm{Q}\right)$ be a metric on $\mathrm{Q}$. Let $\mathcal{W} \subset \mathrm{T}^{*} \mathrm{Q}$ be a subbundle and let $P$ be the associated $\mathbb{G}$-orthogonal projection map as in Section 2. The following set of partial differential equations with $\lambda \in \Gamma^{\omega}\left(\mathrm{T}^{*} \mathrm{Q} \otimes \mathrm{TQ}\right)$ and $\mathbb{B}$ a gyroscopic $(0,3)$-tensor field as dependent variables is called the (extended) $\lambda$-equation:

$$
\nabla_{Z}^{\mathbb{G}}(\mathbb{G} \lambda)(P X, P Y)+\frac{1}{2}\langle\mathbb{B}(\lambda P X, \lambda P Y)+\mathbb{B}(\lambda P Y, \lambda P X), Z\rangle=0,
$$

where $X, Y \in \Gamma^{\omega}(\mathrm{TQ})$.

Definition 4.6. Let $\mathrm{Q}$ be an n-dimensional manifold and let $\mathbb{G} \in \Gamma^{\omega}\left(\mathrm{S}_{2}^{+} \mathrm{T}^{*} \mathrm{Q}\right)$ be a metric on $\mathrm{Q}$. Let $\mathcal{W} \subset \mathrm{T}^{*} \mathrm{Q}$ be a subbundle and $P \in \Gamma^{\omega}\left(\mathrm{T}^{*} \mathrm{Q} \otimes \mathrm{TQ}\right)$ be the 
associated projection map as above. Also let $\lambda \in \Gamma^{\omega}\left(\mathrm{T}^{*} \mathrm{Q} \otimes \mathrm{TQ}\right)$ and let $\mathbb{B}$ be a gyroscopic $(0,3)$-tensor field on $\mathbf{Q}$. The following set of partial differential equations with $\mathbb{G}_{\mathrm{cl}} \in \Gamma^{\omega}\left(\mathrm{S}_{2} \mathrm{~T}^{*} \mathrm{Q}\right)$ as unknown is called the (extended) closed-loop metric equation:

$$
\nabla_{\lambda P X}^{\mathbb{G}} \mathbb{G}_{\mathrm{cl}}(Z, Z)+2 \mathbb{G}_{\mathrm{cl}}\left(\nabla_{Z}^{\mathbb{G}} \lambda P X, Z\right)=2 \mathbb{G}\left(\nabla_{Z}^{\mathbb{G}} P X, Z\right)-2\left\langle\lambda P X, \mathbb{B}^{b}(Z)\right\rangle,
$$

for $X, Y, Z \in \Gamma^{\omega}(\mathrm{TQ})$.

It is clear that the $\lambda$-equation is a first-order linear systems of partial differential equations. The word extended is due to the presence of gyroscopic forces. Theorem 4.3 is an intrinsic version of what has been presented in [4]. Different versions of the proof have been given in $[5,3]$ and modified with presence of gyroscopic forces in [13]. One should note that, by solving the $\lambda$-equations, we only obtain the restriction of $\lambda$ to $\Gamma^{\omega}\left(\mathcal{W}^{\perp} \otimes \mathrm{T}^{*} \mathrm{Q}\right)$, but only this restriction of $\lambda$ appears in the statement of the extended closed-loop metric equation.

REMARK 4.7. Note that there is no assumption on the positive definiteness of the closed-loop metric. In other words, one may very well achieve a closed-loop metric which is not positive definite, but which could possibly lead to a stabilizing energy shaping feedback.

According to Definition 2.2, the energy shaping problem can proceed in two steps: first, kinetic energy shaping and then potential energy shaping. As in [4], we assume that one solves the energy shaping problem in the following steps:

1 . Find the set of pairs $(\lambda, \mathbb{B})$ which satisfy the extended $\lambda$-equations.

2. The system of partial differential equations $(2 \mathrm{~b})$ form Theorem 4.3 with $\mathbb{G}_{\mathrm{cl}}$ as unknown is called the (extended) closed-loop metric system of equations. So we use the set of solutions to the $\lambda$-equations to find a closed-loop metric $\mathbb{G}_{\mathrm{cl}}$ as a solution to the extended closed-loop metric system of equations. This closed-loop metric will be a solution to the kinetic energy shaping problem by the statement of the theorem. Moreover, all the solutions to the kinetic energy shaping problem can be produced by this procedure.

3. Check if the set of $\mathbb{G}_{\mathrm{cl}}$, or equivalently the set of solutions to the $\lambda$-equations satisfies the sufficient condition of potential energy shaping problem, equation (21). If so, one can find a formal solution to the potential energy shaping partial differential equation.

One of our main goals in this paper is to show that a bad choice for the closed-loop metric can make it impossible to find a solution for the energy shaping problem. In order to identify the space of solutions to the energy shaping problem, one should consider together the compatibility conditions of the kinetic energy shaping and potential energy shaping problem. In the coming sections we use the language of geometric partial differential equations to analyze the integrability of the complete set of partial differential equations. 
4.3. The $\lambda$-method partial differential equations. In this section, we formulate the two partial differential equations for the $\lambda$-method in the language of jet bundles. We make the simplifying assumption in this section that $\mathbb{B}=0$. Assume that $\mathcal{W}$ is an $(n-m)$-dimensional integrable subbundle of $T^{*} Q$ where $m$ is the number of unactuated directions.

4.3.1. The equation $\mathbf{R}_{\mathbf{L}}$. With the assumptions above, the set of $\lambda$-equations we consider in this section is

$$
\nabla_{Z}^{\mathbb{G}}(\mathbb{G} \lambda)(P X, P X)=0, \quad X, Z \in \Gamma^{\omega}(\mathrm{TQ}),
$$

where $\lambda \in \Gamma^{\omega}\left(T^{*} Q \otimes T Q\right), \mathbb{G}$ is a metric on $Q$ and $P \in \Gamma^{\omega}\left(T^{*} Q \otimes T Q\right)$ is the $\mathbb{G}$ orthogonal projection as before. We denote by $\mathcal{W}^{\perp}$ the $\mathbb{G}$-orthogonal complement of $\mathcal{W}$ and by $\left.\lambda\right|_{P}$ the restriction of $\lambda$ to $\mathcal{W}^{\perp} \otimes \mathrm{TQ}$. Consider the bundle $\pi: \mathcal{W}^{\perp} \otimes \mathrm{TQ} \rightarrow \mathrm{Q}$ and let $\left(q,\left.\lambda\right|_{P}(q)\right)$ be a typical fiber element over $q \in \mathrm{Q}$. We define the bundle map

$$
\begin{gathered}
\Phi: J_{1} \pi \rightarrow \mathrm{T}^{*} \mathrm{Q} \otimes \mathcal{W}^{\perp} \otimes \mathcal{W}^{\perp} \\
\left.\left.j_{1} \lambda\right|_{P} \mapsto \nabla^{\mathbb{G}}(\mathbb{G} \lambda)\right|_{\operatorname{Im} P^{\otimes 2}},
\end{gathered}
$$

In an adapted coordinate system $\left(q^{i}, \lambda_{a}^{i}\right)$ on $\mathcal{W}^{\perp} \otimes \mathrm{TQ}$ and $\left(q^{i}, \lambda_{a}^{i}, \lambda_{a, k}^{i}\right)$ on $\mathrm{J}^{1} \pi$,

$$
\Phi\left(q^{i}, \lambda_{a}^{i}, \lambda_{a, k}^{i}\right)=\left(q^{i}, \mathbb{G}_{a i} \lambda_{b, k}^{i}+\mathbb{G}_{a i, k} \lambda_{b}^{i}-\mathcal{S}_{k a}^{s} \mathbb{G}_{s i} \lambda_{b}^{i}-\mathcal{S}_{k b}^{s} \mathbb{G}_{s i} \lambda_{a}^{i}\right),
$$

where $\mathcal{S}$ is the coefficient of the Levi-Civita connection associated to $\mathbb{G}$, and $k \in$ $\{1, \cdots, n\}, a, b \in\{1, \cdots, m\}$. Thus we define

$$
\mathrm{R}_{\mathrm{L}} \doteq\left\{p \in \mathrm{J}_{1}(\pi) \mid \Phi(p)=0\right\} .
$$

to be the submanifold of $J_{1} \pi$ corresponding to the $\lambda$-equation.

4.3.2. The equation $R_{\mathbf{E}}$. With the assumptions above, the closed-loop metric equation is

$$
\nabla_{\lambda P X}^{\mathbb{G}} \mathbb{G}_{\mathrm{cl}}(Z, Z)+2 \mathbb{G}_{\mathrm{cl}}\left(\nabla_{Z}^{\mathbb{G}} \lambda P X, Z\right)=2 \mathbb{G}\left(\nabla_{Z}^{\mathbb{G}} P X, Z\right),
$$

for $X, Y, Z \in \Gamma^{\omega}(\mathrm{TQ})$. Consider the bundle $\left(\mathrm{S}_{2} \mathrm{~T}^{*} \mathrm{Q}, \pi, \mathrm{Q}\right)$ and let $\mathcal{W}$ and $P$ be, respectively, the integrable control codistribution and the $\mathbb{G}$-orthogonal projection, respectively, as in Section 2. Let $\lambda$ be an automorphism of TQ and denote a section of $\mathcal{W}^{\perp} \otimes \mathrm{TQ}$ by $\left.\lambda\right|_{P}$. In adapted local coordinates, $\left.\lambda\right|_{P}=\lambda_{a}^{j} e^{a} \otimes e_{j}$, where $a \in$ $\{1, \cdots, m\}$ and $j \in\{1, \cdots, n\}$. Define the bundle map $\Upsilon_{1}: J_{1} \pi \rightarrow \mathrm{T}^{*} \mathrm{Q} \otimes \mathrm{S}_{2} \mathrm{~T}^{*} \mathrm{Q}$ by $\Upsilon_{1}\left(j_{1} \mathbb{G}_{\mathrm{cl}}\right)=\nabla^{\mathbb{G}} \mathbb{G}_{\mathrm{cl}}$. Also define a bundle map

$$
\Upsilon_{0}: S_{2} T^{*} \mathrm{Q} \rightarrow \mathcal{W}^{\perp} \otimes \mathrm{S}_{2} \mathrm{~T}^{*} \mathrm{Q}
$$

by

$$
\Upsilon_{0}(X, Z, Z)=2 \mathbb{G}_{\mathrm{cl}}\left(\nabla_{Z}^{\mathbb{G}} \lambda P X, Z\right)-2 \mathbb{G}\left(\nabla_{Z}^{\mathbb{G}} P X, Z\right)
$$


Let $\Psi_{\mathrm{E}}: \mathrm{T}^{*} \mathrm{Q} \otimes \mathrm{S}_{2} \mathrm{~T}^{*} \mathrm{Q} \rightarrow \mathcal{W}^{\perp} \otimes \mathrm{S}_{2} \mathrm{~T}^{*} \mathrm{Q}$ be the bundle map given in local coordinates by

$$
\Psi_{\mathrm{E}}(\beta \otimes \mathrm{A})=\lambda_{a}^{j} \beta_{j} e^{a} \otimes \mathrm{A},
$$

where $a \in\{1, \cdots, m\}, j \in\{1, \cdots, n\}, \beta \in \Gamma^{\omega}\left(\mathrm{T}^{*} \mathrm{Q}\right)$ and $\mathrm{A} \in \Gamma^{\omega}\left(\mathrm{S}_{2} \mathrm{~T}^{*} \mathrm{Q}\right)$. Observe that the map $\Psi_{\mathrm{E}}$ is surjective. Finally, define $\Phi_{\mathrm{E}}=\Psi_{\mathrm{E}} \circ \Upsilon_{1}+\Upsilon_{0}$. Thus $\mathrm{R}_{\mathrm{E}}=\operatorname{ker} \Phi_{\mathrm{E}}$ gives the extended closed-loop metric system of partial differential equations.

4.4. Potential energy shaping. In this section, we explore aspects of potential energy shaping. Firstly, we recall the result of Lewis [25] regarding potential shaping after kinetic shaping has been done. Then, we couple the sufficient conditions of Lewis [25] with the $\lambda$-equations from the previous section. In this way, we can understand how kinetic energy shaping can influence potential energy shaping.

4.4.1. Sufficient conditions for potential energy shaping. We recall the results for potential energy shaping after kinetic energy shaping from [25]. Denote the bundle automorphism $\mathbb{G}_{\mathrm{ol}}^{b} \circ \mathbb{G}_{\mathrm{cl}}^{\sharp}$ by $\Lambda_{\mathrm{cl}}$. Define an integrable codistribution $\mathcal{W}_{\mathrm{cl}}=$ $\Lambda_{\mathrm{cl}}^{-1}\left(\mathcal{W}_{\mathrm{ol}}\right)$. Let $(\mathrm{PS} \doteq \mathrm{Q} \times \mathbb{R}, \pi, \mathrm{Q})$ be the trivial vector bundle over $\mathrm{Q}$, so that a section of $\pi$ corresponds to a potential function via the formula $q \mapsto(q, V(q))$. We define a $\mathrm{T}^{*} \mathrm{Q}$-valued differential operator $\mathfrak{D}_{\mathrm{d}}(V)=d V$ which induces a vector bundle map $\Phi_{\text {pot }}: J_{1} \pi \rightarrow \mathrm{T}^{*} \mathrm{Q}$ such that $\mathfrak{D}_{\mathrm{d}}(V)(q)=\Phi_{\text {pot }}\left(j_{1} V(q)\right)$. Similar to what we did for kinetic energy shaping we denote by

$$
\pi_{\mathcal{W}_{\mathrm{cl}}}: \mathrm{T}^{*} \mathrm{Q} \rightarrow \mathrm{T}^{*} \mathrm{Q} / \mathcal{W}_{c l}
$$

as the canonical projection.

Definition 4.8. Let $\Sigma_{\mathrm{ol}}=\left(\mathrm{Q}, \mathbb{G}_{\mathrm{ol}}, V_{\mathrm{ol}}, \mathcal{F}_{\mathrm{ol}}, \mathcal{W}_{\mathrm{ol}}\right)$ be an open-loop simple mechanical control system. The submanifold $\mathrm{R}_{\mathrm{pot}} \subset \mathrm{J}_{1} \pi$ defined by

$$
\mathrm{R}_{\mathrm{pot}}=\left\{p \in \mathrm{J}_{1} \pi \mid \pi_{\mathcal{W}_{c l}} \circ \Phi_{\mathrm{pot}}(p)=\pi_{\mathcal{W}_{c l}} \circ \Lambda_{c l}^{-1} d V_{\mathrm{ol}}\right\} .
$$

is called the potential energy shaping submanifold.

Let $\pi_{1}: J_{1} \pi \rightarrow Q$ be the canonical projection. Lewis [25] gives a set of sufficient conditions under which the potential shaping problem has a solution. The proof follows from the integrability theory of partial differential equations; in particular, the potential energy shaping partial differential equation has an involutive symbol. We recall the definition of $\left(\mathbb{G}_{\mathrm{ol}}-\mathbb{G}_{\mathrm{cl}}\right)$-potential energy shaping feedback from [25].

Definition 4.9. A section $\mathcal{F}$ of $\mathcal{W}$ is called a $\left(\mathbb{G}_{\mathrm{ol}}-\mathbb{G}_{\mathrm{cl}}\right)$-potential energy shaping feedback if there exists a function $V_{\mathrm{cl}}$ on $\mathrm{Q}$ such that

$$
\mathcal{F}(q)=\Lambda_{\mathrm{cl}} d V_{\mathrm{cl}}-d V_{\mathrm{ol}}, \quad q \in \mathrm{Q} .
$$

The following results implies when one can construct a Taylor series solution to the potential energy shaping partial differential equation order-by-order. 
THEOREM 4.10. Let $\Sigma_{\mathrm{ol}}=\left(\mathrm{Q}, \mathbb{G}_{\mathrm{ol}}, V_{\mathrm{ol}}, \mathcal{F}_{\mathrm{ol}}, \mathcal{W}_{\mathrm{ol}}\right)$ be an analytic open-loop simple mechanical control system. Let $\mathbb{G}_{\mathrm{cl}}$ be a closed-loop analytic metric. Let $p_{0} \in \mathrm{R}_{\mathrm{pot}}$ and let $q_{0}=\pi_{1}\left(p_{0}\right)$. Assume that $q_{0}$ is a regular point for $\mathcal{W}_{\mathrm{ol}}$ and that $\mathcal{W}_{c l}=\Lambda_{\mathrm{cl}}^{-1} \mathcal{W}_{\mathrm{ol}}$ is integrable in a neighborhood of $q_{0}$. Then the following statements are equivalent:

1. there exists a neighborhood $U$ of $q_{0}$ and an analytic $\left(\mathbb{G}_{\mathrm{ol}}-\mathbb{G}_{\mathrm{cl}}\right)$-potential energy shaping feedback $\mathcal{F} \in \Gamma^{\omega}(\mathcal{W})$ defined on $U$ which satisfies

$$
\Phi_{\mathrm{pot}}\left(p_{0}\right)=\Lambda_{\mathrm{cl}} d V\left(q_{0}\right)-d V_{\mathrm{ol}}\left(q_{0}\right)+\Lambda_{\mathrm{cl}}^{-1} d V_{\mathrm{ol}}\left(q_{0}\right),
$$

for a solution $V$ to $\mathrm{R}_{\mathrm{pot}}$;

2. there exists a neighborhood $U$ of $q_{0}$ such that $d\left(\Lambda_{\mathrm{cl}}^{-1} d V_{\mathrm{ol}}\right)(q) \in \mathrm{I}_{2}\left(\left.\mathcal{W}_{c l}\right|_{q}\right)$, where we denote $\mathrm{I}_{2}\left(\left.\mathcal{W}_{c l}\right|_{q}\right)=\mathrm{I}\left(\left.\mathcal{W}_{c l}\right|_{q}\right) \cap \Lambda_{2}\left(\mathrm{~T}_{q}^{*} \mathrm{Q}\right)$ where the algebraic ideal $\mathrm{I}\left(\left.\mathcal{W}_{c l}\right|_{q}\right)$ of $\Lambda\left(\mathrm{T}_{q}^{*} \mathrm{Q}\right)$ is generated by elements of the form $\gamma \wedge \omega$ where $\left.\gamma \in \mathcal{W}_{c l}\right|_{q}$.

The theorem gives a set of compatibility conditions for the existence of a $\left(\mathbb{G}_{\mathrm{ol}^{-}}\right.$ $\mathbb{G}_{\mathrm{cl}}$ )-potential energy shaping feedback. Moreover, one can give a full description of the set of achievable potential energy shaping feedbacks. Let $\alpha_{c l}=\Lambda_{\mathrm{cl}}^{-1} d V_{\mathrm{ol}}$. Let us use a coordinate system $\left(q^{1}, \cdots, q^{n}\right)$ on $U$ a neighborhood of $q_{0}$ such that

$$
\left.\mathcal{W}_{c l}\right|_{q_{0}}=\operatorname{Span}\left(d q^{m+1}, \cdots, d q^{n}\right) .
$$

In these local coordinate we write the one form $\alpha_{c l}$ as $\alpha_{c l}=\alpha_{j} d q^{j}$ and compatibility conditions become:

$$
\frac{\partial \alpha_{j}}{\partial q^{i}}-\frac{\partial \alpha_{i}}{\partial q^{j}}=0 \quad, \quad i, j \in\{1, \cdots, m\} .
$$

REMARK 4.11. One can make the following observations from the potential energy shaping problem.

1. The choice of $\mathbb{G}_{\mathrm{cl}}$ affects the set of solutions that one might get for potential energy shaping. A bad choice of $\mathbb{G}_{c l}$ might make it impossible to find any potential energy shaping feedback. As a result, if one is able to have an understanding of the set of closed-loop energy shaping metrics, then the condition given by equation (21) is an obstruction that detects the closedloop energy shaping metrics for which their exists a potential energy shaping feedback. We give a complete description of this problem in Section 7.

2. Following [24], if we denote the set of all solutions for the potential shaping problem by

$\mathrm{PS}_{q_{0}}=\left\{V_{\mathrm{cl}} \oplus \mathcal{F}_{\mathrm{cl}} \in C^{\omega}(\mathrm{Q}) \oplus \Gamma^{\omega}\left(\mathcal{W}_{\mathrm{cl}}\right) \mid d V_{\mathrm{cl}}=\mathcal{F}_{\mathrm{cl}}+\Lambda_{\mathrm{cl}}^{-1} d V_{\mathrm{ol}} \quad, \quad V_{\mathrm{cl}}\left(q_{0}\right)=0\right\}$, one can describe the whole set of solutions as an affine subspace of $C^{\omega}(\mathrm{Q}) \oplus$ $\Gamma^{\omega}\left(\mathcal{W}_{c l}\right)$ modeled on the subspace

$$
L\left(\mathrm{PS}_{q_{0}}\right)=\left\{f \oplus \beta \in C^{\omega}(\mathrm{Q}) \oplus \Gamma^{\omega}\left(\mathcal{W}_{c l}\right) \mid d f=\beta\right\} .
$$


4.4.2. The equation $R_{T}$. The sufficient condition for integrability of the partial differential equation in potential energy shaping, given in equation (21), is a nonlinear partial differential equation with the dependent variable $\Lambda=\mathbb{G}_{\mathrm{ol}}^{b} \circ \mathbb{G}_{\mathrm{cl}}^{\sharp}$. The following commutative diagram shows the relation between $\lambda$ and $\Lambda$ :

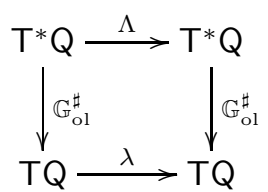

Any condition on $\Lambda$ imposes conditions on $\lambda$ and vice versa. Through these conditions, we can find the obstruction that the potential energy shaping integrability condition imposes on the set of solutions to the $\lambda$-equations. For more information about the procedure we propose for energy shaping, see Section 8. We make some algebraic constructions before moving to the potential energy shaping problem.

An algebraic construction Let $\mathrm{V}$ be a finite-dimensional $\mathbb{R}$-vector space and denote the dual vector space by $\mathrm{V}^{*}$. Let $\mathrm{D}$ be a subspace of $\mathrm{V}^{*}$. The algebraic ideal I(D) of $\Lambda\left(V^{*}\right)$ is generated by elements of the form $\gamma \wedge \omega$ where $\gamma \in \mathrm{D}$. For $k \in \mathbb{Z}$ we denote $\mathrm{I}_{k}(\mathrm{D})=\mathrm{I}(\mathrm{D}) \cap \Lambda_{k}\left(\mathrm{~V}^{*}\right)$. For $\Theta \in \operatorname{Aut}\left(\mathrm{V}^{*}\right)$, we wish to understand $\mathrm{I}_{2}(\Theta(\mathrm{D}))$.

Lemma 4.12. We have $\mathrm{I}_{2}(\Theta(\mathrm{D}))=(\Theta \otimes \Theta)\left(\mathrm{I}_{2}(\mathrm{D})\right)$.

Proof. Let $\left\{v^{1}, \cdots, v^{n}\right\}$ be a basis for $\mathrm{V}$, and suppose that

$$
\mathrm{D}=\operatorname{Span}\left\{v^{m+1}, v^{m+2}, \cdots, v^{n}\right\} .
$$

One can identify $\mathrm{I}_{2}(\Theta(\mathrm{D}))$ by

$$
\mathrm{I}_{2}(\Theta(\mathrm{D}))=\operatorname{Span}\left\{\Theta\left(v^{j}\right) \wedge \Theta\left(v^{i}\right) \mid m+1 \leq j \leq n, 1 \leq i \leq n\right\},
$$

If one extends $\Theta$ to $\Theta \otimes \Theta$ on $\mathrm{V}^{*} \otimes \mathrm{V}^{*}$ in the usual way we have

$$
\Theta \otimes \Theta\left(\mathrm{I}_{2}(\mathrm{D})\right)=\operatorname{Span}\left\{\Theta\left(v^{j}\right) \wedge \Theta\left(v^{i}\right) \mid m+1 \leq j \leq n, 1 \leq i \leq n\right\}
$$

as desired.

LEMma 4.13. Let $\alpha$ be an analytic local section of $\left(\mathrm{T}^{*} \mathrm{Q}, \pi, \mathrm{Q}\right)$ in a neighborhood $U$ of $\mathrm{Q}$ and let $\mathrm{D} \subset \mathrm{T}^{*} \mathrm{Q}$ be a subbundle. Then $\mathrm{d} \alpha \in \mathrm{I}_{2}(\Theta(\mathrm{D}))$ if and only if

$$
\Theta^{-1} \otimes \Theta^{-1}(\mathrm{~d} \alpha) \in \mathrm{I}_{2}(\mathrm{D})
$$

Proof. Note that $(\Theta \otimes \Theta)^{-1}=\Theta^{-1} \otimes \Theta^{-1}$ and so the proof follows from the previous lemma.

Proposition 4.14. Let $\mathrm{Q}$ be an $n$-dimensional manifold and let $\beta \neq 0$ and $\alpha$ be analytic local sections of $\mathrm{T}^{*} \mathrm{Q}$ such that $\alpha=\Theta(\beta)$, where $\Theta \in \operatorname{Aut}\left(\mathrm{T}^{*} \mathrm{Q}\right)$. Let $U$ be a neighborhood of $p \in \mathrm{Q}$. Given $\mathrm{D}$, an integrable subbundle of $\mathrm{T}^{*} \mathrm{Q}$, with adapted local 
coordinates $\left\{d q^{m+1}, \cdots, d q^{n}\right\}$ as above, we have $\mathrm{d} \alpha \in \mathrm{I}_{2}(\Theta(\mathrm{D}))$ in this neighborhood if and only if

$$
\left(\Delta_{i}^{k} \Delta_{j}^{p}-\Delta_{j}^{k} \Delta_{i}^{p}\right)\left(\frac{\partial \Theta_{k}^{l}}{\partial x^{p}} \beta_{l}+\Theta_{k}^{l} \frac{\partial \beta_{l}}{\partial x^{p}}\right)=0,
$$

where $i, j, k \in\{1, \cdots, m\}$ and we denoted the $\Theta^{-1}$ by $\Delta$.

Proof. Using Lemma 4.13 we have

$$
\mathrm{d} \alpha=\Delta_{i}^{k} \Delta_{j}^{p}\left(\frac{\partial \Theta_{k}^{l}}{\partial x^{p}} \beta_{l}+\Theta_{k}^{l} \frac{\partial \beta_{l}}{\partial x^{p}}\right) d q^{i} \wedge d q^{j} .
$$

The proof follows since $\Lambda_{2} T^{*} \mathrm{Q}=\mathrm{I}_{2}(\mathrm{D}) \oplus \Lambda_{2}\left(\mathrm{D}^{\perp}\right)$.

Partial differential equation We consider the system of partial differential equations of Proposition 4.14 with the automorphism $\Theta$ as unknown. One can easily observe that this system of partial differential equation is equivalent to the system of partial differential equations one would obtain by assuming equation (21) as a partial differential equation with unknown $\Lambda^{-1}$. We consider nonlinear partial differential equations as described briefly in Section 3.1. More details on the formal integrability of nonlinear systems of partial differential equations can be found in [19].

Let $(\mathrm{Q}, \mathbb{G})$ be an $n$-dimensional Riemannian manifold and let $\mathcal{W} \subset \mathrm{T}^{*} \mathrm{Q}$ be a subbundle. Consider the vector bundle $\left(\pi,\left(\mathcal{W}^{\perp} \otimes_{\mathrm{Q}} \mathrm{TQ}\right) \oplus\left(\mathcal{W}^{\perp} \otimes_{\mathrm{Q}} \mathrm{TQ}\right), \mathrm{Q}\right)$ with a typical fiber $(q, \Theta(q) \oplus \Delta(q))$ and denote its first jet bundle by $\mathrm{J}_{1} \pi$. We define the following system of partial differential equations in a neighborhood $U$ of $q_{0} \in \mathrm{Q}$ :

$$
\mathrm{R}_{\mathrm{T}}=\left\{j_{1}(\Theta \oplus \Delta) \in \mathrm{J}_{1} \pi \mid \Phi_{\mathrm{T}}\left(j_{1}(\Theta \oplus \Delta)\right)=0\right\}
$$

where $\Phi_{\mathrm{T}}$ can be written in adapted local coordinates

$$
\Phi_{\mathrm{T}}\left(j_{1}(\Theta \oplus \Delta)\right)=\left(\Delta_{i}^{k} \Delta_{j}^{r}-\Delta_{j}^{k} \Delta_{i}^{r}\right)\left(\frac{\partial \Theta_{k}^{l}}{\partial q^{r}} \beta_{l}+\Theta_{k}^{l} \frac{\partial \beta_{l}}{\partial q^{r}}\right),
$$

where $i, j, k \in\{1, \cdots, m\}$ and $\beta \in \Gamma^{\omega}\left(\mathrm{T}^{*} \mathrm{Q}\right)$. This system of partial differential equations is quasi-linear and so we need to use Definition 3.7 to find the symbol. We look at the linearization of the partial differential equation about a given reference point. Let $(q, \bar{\Theta}(q) \oplus \bar{\Delta}(q))$ be a typical fiber of $\pi$. If we linearize the system about this point we have

$$
\begin{aligned}
\left.\frac{d}{d t}\right|_{t=0} & \left(\left(\left(\Delta_{i}^{k} t+\bar{\Delta}_{i}^{k}\right)\left(\Delta_{j}^{p} t+\bar{\Delta}_{j}^{r}\right)-\left(\Delta_{j}^{k} t+\bar{\Delta}_{j}^{k}\right)\left(\Delta_{i}^{p} t+\bar{\Delta}_{i}^{r}\right)\right)\right. \\
& \left.\times\left[\frac{\partial\left(\Theta_{k}^{l} t+\bar{\Theta}_{k}^{l}\right)}{\partial q^{r}} \beta_{l}+\left(\Theta_{k}^{l} t+\bar{\Theta}_{k}^{l}\right) \beta_{l}\right)\right) \\
& =\left(\Delta_{i}^{k} \bar{\Delta}_{j}^{r}+\Delta_{j}^{r} \bar{\Delta}_{i}^{k}-\Delta_{j}^{k} \bar{\Delta}_{i}^{r}-\Delta_{i}^{r} \bar{\Delta}_{j}^{k}\right) \frac{\partial \bar{\Theta}_{k}^{l}}{\partial q^{r}} \beta_{l} \\
& +\left(\bar{\Delta}_{i}^{k} \bar{\Delta}_{j}^{r}-\bar{\Delta}_{j}^{k} \bar{\Delta}_{i}^{r}\right)\left(\frac{\partial \Theta_{k}^{l}}{\partial q^{r}} \beta_{l}+\Theta_{k}^{l} \frac{\partial \beta_{l}}{\partial q^{r}}\right) .
\end{aligned}
$$


The effect on the reference point of the linearization should be investigated carefully. For now, we consider the linearization of the system about a point $p \in \mathrm{J}_{1} \pi$ with

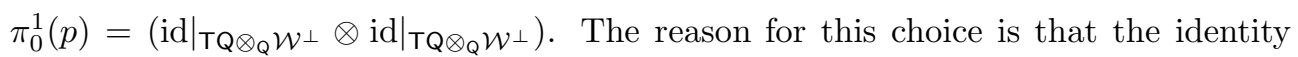
solution for $\Theta$ refers to the open-loop system which is always a solution to the energy shaping problem. Thus we study the linearization of the nonlinear system about the open-loop solution. We have the following linearization of $\Phi_{\mathrm{T}}$ about $p$ :

$$
\mathrm{V}_{p}\left(\Phi_{\mathrm{T}}\right)\left(j_{1}(\Theta \oplus \Delta)\right)=\left(\frac{\partial \Theta_{i}^{l}}{\partial q^{j}} \beta_{l}-\frac{\partial \Theta_{j}^{l}}{\partial q^{i}} \beta_{l}\right)+\left(\Theta_{i}^{l} \frac{\partial \beta_{l}}{\partial q^{j}}-\Theta_{j}^{l} \frac{\partial \beta_{l}}{\partial q^{i}}\right),
$$

where we utilized the fact that $\mathrm{VJ}_{1} \pi \cong \mathrm{J}_{1} \vee \pi[27]$

5. Formal integrability of $R_{\mathbf{L}}$. In this section, we apply the theorem of Goldschmidt, Theorem 3.20, to the $\lambda$-equations. The main result in this section is Theorem 5.6 which gives sufficient conditions for formal integrability of the $\lambda$-equations. The proof of this theorem requires the machinery of Section 3.1. However, the main result can be understood without understanding the details of the proof.

5.1. The symbol of $R_{\mathbf{L}}$. The symbol map $\sigma\left(R_{L}\right): T^{*} Q \otimes \mathcal{W}^{\perp} \otimes T Q \rightarrow T^{*} Q \otimes$ $\mathcal{W}^{\perp} \otimes \mathcal{W}^{\perp}$ is defined by

$$
\sigma\left(\mathrm{R}_{\mathrm{L}}\right)(\mathrm{A})(X, P Y, P Z)=\mathrm{A}\left(X, P Y, \mathbb{G}^{b}(P Z)\right), \quad \mathrm{A} \in \Gamma^{\omega}\left(\mathrm{T}^{*} \mathrm{Q} \otimes \mathcal{W}^{\perp} \otimes \mathrm{TQ}\right),
$$

where $X, Y, Z \in \Gamma^{\omega}(\mathrm{TQ})$. This can be shown using the affine structure of $\mathrm{J}_{1} \pi$ as follows. Take $p_{1}, p_{2} \in \mathrm{J}_{1} \pi$ such that $\pi_{0}^{1}\left(p_{1}\right)=\pi_{0}^{1}\left(p_{2}\right)$. Then $p_{1}-p_{2} \in \Gamma^{\omega}\left(\mathbf{T}^{*} \mathrm{Q} \otimes\right.$ $\mathcal{W}^{\perp} \otimes \mathrm{TQ}$ ) by the affine structure of $J_{1} \pi$. Now one can define the symbol map to be $\Phi\left(p_{2}-p_{1}\right)$. Using equation (20), one can observe in local coordinates that $\Phi\left(p_{1}-p_{2}\right)$ is the highest order term of the partial differential equations since $\pi_{0}^{1}\left(p_{1}\right)=\pi_{0}^{1}\left(p_{2}\right)$. Let us determine the symbol $G_{1}\left(R_{L}\right)$ and its prolongation.

LEMMA 5.1. The following sequence is short exact:

$$
0 \longrightarrow \mathrm{G}_{1}\left(\mathrm{R}_{\mathrm{L}}\right) \longrightarrow \mathrm{T}^{*} \mathrm{Q} \otimes \mathcal{W}^{\perp} \otimes \mathrm{TQ} \stackrel{\sigma\left(\mathrm{R}_{\mathrm{L}}\right)}{\longrightarrow} \mathrm{T}^{*} \mathrm{Q} \otimes \mathcal{W}^{\perp} \otimes \mathcal{W}^{\perp} \longrightarrow 0
$$

Proof. The symbol map is surjective since $\sigma\left(\mathrm{R}_{\mathrm{L}}\right)=\mathrm{id}_{\mathrm{T}^{*} \mathrm{Q} \otimes \mathcal{W}^{\perp}} \otimes\left(\mathbb{G}^{b} \circ P\right)$.

Let $\left\{e^{1}, \cdots, e^{n}\right\}$ be a basis for $\mathrm{T}_{q_{0}}^{*} \mathrm{Q}$ for $q_{0} \in \mathrm{Q}$ and let $\Sigma_{j}$ be the subspace of $\mathbf{T}_{q_{0}}^{*} \mathbf{Q}$ generated by $\left\{e^{j+1}, \cdots, e^{n}\right\}$. Then we have the following lemma, similar to Lemma 5.1.

LEMmA 5.2. The following sequence is short exact:

$$
0 \longrightarrow \mathrm{G}_{1, j}\left(\mathrm{R}_{\mathrm{L}}\right) \longrightarrow \Sigma_{j} \otimes \mathcal{W}^{\perp} \otimes \mathrm{TQ} \stackrel{\sigma\left(\mathrm{R}_{\mathrm{L}}\right)}{\longrightarrow} \Sigma_{j} \otimes \mathcal{W}^{\perp} \otimes \mathcal{W}^{\perp} \longrightarrow 0
$$

where $\mathrm{G}_{1, j}\left(\mathrm{R}_{\mathrm{L}}\right)=\mathrm{G}_{1}\left(\mathrm{R}_{\mathrm{L}}\right) \cap \Sigma_{j}$.

The following lemma characterizes the prolonged symbol $\rho_{1}\left(G_{1}\left(R_{L}\right)\right)$. 
LEMma 5.3. The following sequence is short exact:

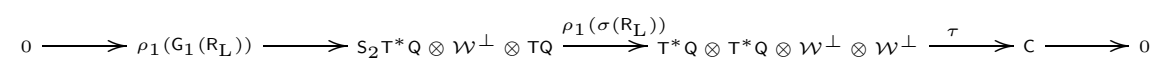

where $\mathrm{C} \cong \Lambda^{2} \mathrm{~T}^{*} \mathrm{Q} \otimes \mathcal{W}^{\perp} \otimes \mathcal{W}^{\perp}$ and $\tau$ is given by

$$
\tau(\mathrm{A})\left(X_{1}, X_{2}, Y_{1}, Y_{2}\right)=\mathrm{A}\left(X_{1}, X_{2}, Y_{1}, Y_{2}\right)-\mathrm{A}\left(X_{2}, X_{1}, Y_{1}, Y_{2}\right) .
$$

Proof. Note that $\tau$ is the alternation map up to a constant coefficient and so is surjective. Moreover, we have

$$
\rho_{1}\left(\mathrm{G}_{1}\left(\mathrm{R}_{\mathrm{L}}\right)\right)(\mathrm{A})\left(X_{1}, X_{2}, Y_{1}, Y_{2}\right)=\mathrm{A}\left(X_{1}, X_{2}, Y_{1}, \mathbb{G}^{b} Y_{2}\right),
$$

as a consequence of the fact that $\tau \circ \rho_{1}\left(\mathrm{G}_{1}\left(\mathrm{R}_{\mathrm{L}}\right)\right)$ is zero since $\mathrm{A}$ is symmetric in the first two elements.

LEMMA 5.4. The symbol $\mathrm{G}_{1}\left(\mathrm{R}_{\mathrm{L}}\right)$ is involutive.

Proof. We will show that the basis $\left\{e^{1}, \cdots, e^{n}\right\}$ is a quasi-regular basis. This is just a dimension count. From Lemmata 5.1 and 5.2 we have

$$
\begin{aligned}
\operatorname{dim}\left(\mathrm{G}_{1}\left(\mathrm{R}_{\mathrm{L}}\right)\right) & =n m(n-m), \\
\operatorname{dim}\left(\mathrm{G}_{1, j}\left(\mathrm{R}_{\mathrm{L}}\right)\right) & =(n-j) m(n-m) .
\end{aligned}
$$

We compute

$$
\operatorname{dim}\left(\mathrm{G}_{1}\left(\mathrm{R}_{\mathrm{L}}\right)\right)+\sum_{j=1}^{n-1} \operatorname{dim}\left(\mathrm{G}_{1, j}\left(\mathrm{R}_{\mathrm{L}}\right)\right)=\frac{1}{2} n m(n+1)(n-m),
$$

which is precisely dimension of $\rho_{1}\left(\mathrm{G}_{1}\left(\mathrm{R}_{\mathrm{L}}\right)\right)$ using Lemma 5.3.

\subsection{Involutivity of $\mathbf{R}_{\mathbf{L}}$.}

TheOREM 5.5. The set of $\lambda$-equations $\mathrm{R}_{\mathrm{L}}$ is involutive if, for $p \in \mathrm{R}_{\mathrm{L}}$, we have

$$
\tau\left(\rho_{1}(\Phi)\left(p_{2}\right)-0\right)=0
$$

where $p_{2}$ is any point in $\mathrm{J}_{2}(\pi)$ that projects to $p$.

Proof. Note that $\rho_{1}\left(G_{1}\left(R_{L}\right)\right)$ is isomorphic to $S_{2} T^{*} Q \otimes \mathcal{W}^{\perp} \otimes \mathbb{G}^{\sharp} \mathcal{W}$. Therefore, it is a vector bundle on the open subset for which $\mathbb{G}^{\sharp} \mathcal{W}$ is a vector bundle. Let $\mathrm{C}$ be the cokernel of $\left.\rho_{1}(\sigma(\Phi))\right)$. Then $\mathrm{G}\left(\mathrm{R}_{\mathrm{L}}\right)$ is involutive and so the system of partial differential equations $R_{L}$ is involutive if the curvature map $\kappa: R_{L} \rightarrow C$, defined as in equation (4), is zero. We have the following exact commutative diagram:

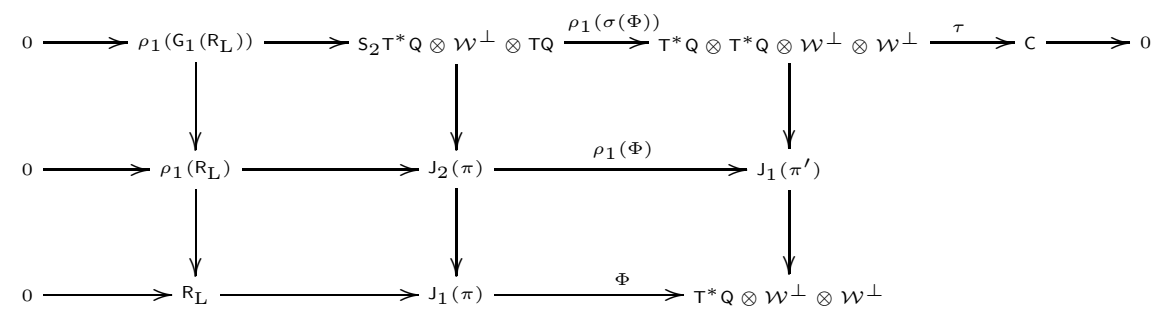


Let $p \in \mathrm{R}_{\mathrm{L}}$ so that $\pi(p)=q$ for $q \in \mathrm{Q}$. Therefore, $\Phi(p)=0$. Take $p_{2} \in \mathrm{J}_{2}(\pi)$ projecting to $p$ and define $\xi=\rho_{1}(\Phi)\left(p_{2}\right) \in \mathrm{J}_{1}\left(\pi^{\prime}\right)$. By commutativity of the diagram, $\xi$ projects to $0 \in \mathrm{T}^{*} \mathrm{Q} \otimes \mathcal{W}^{\perp} \otimes \mathcal{W}^{\perp}$, so we take $\kappa(p)=\tau(\xi-0)$. It is easy to show that the definition of $\kappa$ is independent of the choice of $p_{2}[27,28]$. By the discussion in Section $3.1, p$ is in the image of the projection of $\rho_{1}\left(\mathrm{R}_{\mathrm{L}}\right)$ to $\mathrm{R}_{\mathrm{L}}$ if and only if $\kappa(p)=0$.

Using Theorem 5.5 and the map defined by equation (24), we can write the following intrinsic formula for $\kappa$ :

$$
\begin{aligned}
& \kappa\left(j_{1} \lambda\right)(Z, W, P X, P Y)= \\
& \nabla_{W}^{\mathbb{G}}\left[\nabla_{Z}^{\mathbb{G}}(\mathbb{G} \lambda)(P X, P X)\right]-\nabla_{Z}^{\mathbb{G}}\left[\nabla_{W}^{\mathbb{G}}(\mathbb{G} \lambda)(P X, P X)\right]-\nabla_{[W, Z]}^{\mathbb{G}}(\mathbb{G} \lambda)(P X, P X) .
\end{aligned}
$$

This leads to the following theorem which gives an explicit expression for the compatibility conditions of the $\lambda$-equations.

TheOrem 5.6. Let $(\mathrm{Q}, \mathbb{G})$ be an analytic Riemannian manifold of dimension $n$ and let $\mathcal{S}$ be the Levi-Civita connection on $\mathrm{Q}$ with the associated curvature tensor R. Let $\mathcal{W} \subset \mathrm{T}^{*} \mathrm{Q}$ be a given analytic subbundle and let $P \in \Gamma^{\omega}\left(\mathrm{T}^{*} \mathrm{Q} \otimes \mathrm{TQ}\right)$ be the associated $\mathbb{G}$-orthogonal projection as above. If the partial differential equation

$$
\begin{aligned}
& (\mathbb{G} \lambda)(\mathrm{R}(P X, P X) W, Z)+(\mathbb{G} \lambda)(W, \mathrm{R}(P X, P X) Z)+ \\
& \left.2 \nabla_{Z}^{\mathbb{G}}(\mathbb{G} \lambda)\left(\nabla_{W}^{\mathbb{G}} P X, P X\right)-2 \nabla_{W}^{\mathbb{G}}(\mathbb{G} \lambda)\left(\nabla_{Z}^{\mathbb{G}} P X, P X\right)\right)=0
\end{aligned}
$$

is satisfied in a neighborhood of $\lambda_{0} \in \Gamma^{\omega}\left(\mathrm{T}^{*} \mathrm{Q} \otimes \mathrm{TQ}\right)$, then the set of $\lambda$-equations has a solution in a neighborhood of $\lambda_{0}$. Moreover, any solution to the $\lambda$-equations will satisfy equation (26).

Proof. From Theorem 5.5 and involutivity of the symbol of $\mathrm{R}_{\mathrm{L}}$, a sufficient condition for the existence of solutions to the $\lambda$-equations is that the curvature map be zero. We have

$$
\nabla_{Z}^{\mathbb{G}}(\mathbb{G} \lambda)(P X, P X)=\mathbb{G}\left(\left(\nabla_{Z}^{\mathbb{G}} \lambda\right)(P X), P X\right),
$$

for all $X, Z \in \Gamma^{\omega}(\mathrm{TQ})$. Thus

$$
\begin{aligned}
\nabla_{W}^{\mathbb{G}}\left[\nabla_{Z}^{\mathbb{G}}(\mathbb{G} \lambda)(P X, P X)\right] & =\nabla_{W}^{\mathbb{G}}\left[\mathbb{G}\left(\left(\nabla_{Z}^{\mathbb{G}} \lambda\right)(P X), P X\right)\right] \\
& =\mathbb{G}\left(\nabla_{W}^{\mathbb{G}}\left(\nabla_{Z}^{\mathbb{G}} \lambda(P X)\right), P X\right)+\mathbb{G}\left(\nabla_{Z}^{\mathbb{G}} \lambda(P X), \nabla_{W}^{\mathbb{G}} P X\right),
\end{aligned}
$$

where $X, Z, W \in \Gamma^{\omega}(\mathrm{TQ})$. Therefore,

$$
\nabla_{W}^{\mathbb{G}}\left[\nabla_{Z}^{\mathbb{G}}(\mathbb{G} \lambda)(P X, P X)\right]=\mathbb{G}\left(\nabla_{W}^{\mathbb{G}}\left(\nabla_{Z}^{\mathbb{G}} \lambda(P X)\right), P X\right)+\mathbb{G}\left(\nabla_{Z}^{\mathbb{G}} \lambda(P X), \nabla_{W}^{\mathbb{G}} P X\right) .
$$

As a result,

$$
\begin{aligned}
& \nabla_{W}^{\mathbb{G}}\left[\nabla_{Z}^{\mathbb{G}}(\mathbb{G} \lambda)(P X, P X)\right] \\
& =\mathbb{G}\left(\nabla_{W}^{\mathbb{G}} \nabla_{Z}^{\mathbb{G}} \lambda(P X), P X\right)+2 \nabla_{Z}^{\mathbb{G}}(\mathbb{G} \lambda)\left(\nabla_{W}^{\mathbb{G}} P X, P X\right) .
\end{aligned}
$$


We conclude that

$$
\begin{aligned}
& \nabla_{W}^{\mathbb{G}}\left[\nabla_{Z}^{\mathbb{G}}(\mathbb{G} \lambda)(P X, P X)\right]-\nabla_{Z}^{\mathbb{G}}\left[\nabla_{W}^{\mathbb{G}}(\mathbb{G} \lambda)(P X, P X)\right]-\nabla_{[W, Z]}^{\mathbb{G}}(\mathbb{G} \lambda)(P X, P X) \\
& =\mathbb{G}\left(\nabla_{W}^{\mathbb{G}} \nabla_{Z}^{\mathbb{G}} \lambda(P X), P X\right)-\mathbb{G}\left(\nabla_{Z}^{\mathbb{G}} \nabla_{W}^{\mathbb{G}} \lambda(P X), P X\right)-\mathbb{G}\left(\nabla_{[W, Z]}^{\mathbb{G}} \lambda(P X), P X\right)+ \\
& \quad+2 \nabla_{Z}^{\mathbb{G}}(\mathbb{G} \lambda)\left(\nabla_{W}^{\mathbb{G}} P X, P X\right)-2 \nabla_{W}^{\mathbb{G}}(\mathbb{G} \lambda)\left(\nabla_{Z}^{\mathbb{G}} P X, P X\right) .
\end{aligned}
$$

Finally, using the Ricci identity and Theorem 5.5, we have the following sufficient condition for the existence of solutions:

$$
\begin{aligned}
& (\mathbb{G} \lambda)(\mathrm{R}(P X, P X) W, Z)+(\mathbb{G} \lambda)(W, \mathrm{R}(P X, P X) Z) \\
& \quad+2 \nabla_{Z}^{\mathbb{G}}(\mathbb{G} \lambda)\left(\nabla_{W}^{\mathbb{G}} P X, P X\right)-2 \nabla_{W}^{\mathbb{G}}(\mathbb{G} \lambda)\left(\nabla_{Z}^{\mathbb{G}} P X, P X\right)=0 .
\end{aligned}
$$

The necessity of this condition is clear since any solution of the $\lambda$-equation satisfies equation (25) by definition.

6. Formal integrability of $R_{\mathbf{E}}$. We prove that the system of partial differential equations for the closed-loop metric has an involutive symbol and is formally integrable under a certain surjectivity condition. An additional assumption is that $\lambda\left(\mathrm{TQ} /\left(\mathbb{G}_{\mathrm{ol}}^{\sharp} \mathcal{W}\right)\right)$ is integrable. Recall that a similar assumption has been used in Theorem 4.10. The main result here is Theorem 6.6. Again, this result can be understood separately from the details of its proof.

6.1. The symbol of $R_{\mathbf{E}}$. We have the symbol map $\sigma\left(R_{E}\right): T^{*} Q \otimes S_{2} T^{*} Q \rightarrow$ $\mathcal{W}^{\perp} \otimes \mathrm{S}_{2} \mathrm{~T}^{*} \mathrm{Q}$ for the partial differential equation $\mathrm{R}_{\mathrm{E}}$ given by

$$
\sigma\left(\mathrm{R}_{\mathrm{E}}\right)(\beta \otimes \mathrm{A})=\lambda_{a}^{j} \beta_{j} e^{a} \otimes \mathrm{A}
$$

in local coordinates.

Lemma 6.1. We have $\mathrm{G}\left(\mathrm{R}_{\mathrm{E}}\right) \doteq \operatorname{ker}\left(\sigma\left(\mathrm{R}_{\mathrm{E}}\right)\right) \cong \mathcal{W} \otimes \mathrm{S}_{2} \mathrm{~T}^{*} \mathrm{Q}$.

Proof. Note that $\lambda$ is an isomorphism, so $\operatorname{ker}\left(\sigma\left(\mathrm{R}_{\mathrm{E}}\right)\right)$ is of dimension $(n-m) \times$ $(n \times(n+1) / 2)$ and so is isomorphic to $\mathcal{W} \otimes \mathrm{S}_{2} \mathrm{~T}^{*} \mathrm{Q}$ as claimed.

Let $\left\{e^{1}, e^{2}, \cdots, e^{n}\right\}$ be a basis for $\mathrm{T}_{q_{0}}^{*} \mathrm{Q}$ such that $\left\{e^{1}, \cdots, e^{n-m}\right\}$ spans $\mathcal{W}$ and let $\Sigma_{j}$ be the subspace of $\mathrm{T}_{q_{0}}^{*} \mathrm{Q}$ generated by $\left\{e^{j+1}, \cdots, e^{n}\right\}$. Consider the restriction

$$
\left.\sigma\left(\mathrm{R}_{\mathrm{E}}\right)\right|_{\Sigma_{j}}: \Sigma_{j} \otimes \mathrm{S}_{2} \mathrm{~T}^{*} \mathrm{Q} \rightarrow \mathcal{W}^{\perp} \otimes \mathrm{S}_{2} \mathrm{~T}^{*} \mathrm{Q},
$$

of the symbol map to $\Sigma_{j} \otimes \mathrm{S}_{2} \mathrm{~T}^{*} \mathrm{Q}$. We have the following lemma:

Lemma 6.2. We have

$$
\mathrm{G}\left(\mathrm{R}_{\mathrm{E}}\right)_{1, j} \doteq \operatorname{ker}\left(\left.\sigma\left(\mathrm{R}_{\mathrm{E}}\right)\right|_{\Sigma_{j} \otimes \mathrm{S}_{2} \mathrm{~T}^{*} \mathrm{Q}}\right)=\left(\Sigma_{j} \cap \mathcal{W}\right) \otimes \mathrm{S}_{2} \mathrm{~T}^{*} \mathrm{Q} .
$$

Proof. The proof follows along the same lines as that of Lemma 6.1.

In local coordinates, the prolongation of the symbol map

$$
\rho_{1}\left(\sigma\left(\mathrm{R}_{\mathrm{E}}\right)\right): \mathrm{S}_{2} \mathrm{~T}^{*} \mathrm{Q} \otimes \mathrm{S}_{2} \mathrm{~T}^{*} \mathrm{Q} \rightarrow \mathrm{T}^{*} \mathrm{Q} \otimes \mathcal{W}^{\perp} \otimes \mathrm{S}_{2} \mathrm{~T}^{*} \mathrm{Q}
$$


is given by

$$
\rho_{1}\left(\sigma\left(\mathrm{R}_{\mathrm{E}}\right)\right)(\Pi \otimes \mathrm{A})=\lambda_{a}^{j} \Pi_{j k} \otimes \mathrm{A},
$$

where $\Pi \in \Gamma^{\omega}\left(\mathrm{S}_{2} \mathrm{~T}^{*} \mathrm{Q}\right)$.

Lemma 6.3. The following sequence is short exact:

$$
\mathrm{S}_{2} \mathrm{~T}^{*} \mathrm{Q} \otimes \mathrm{S}_{2} \mathrm{~T}^{*} \mathrm{Q} \stackrel{\rho_{1}\left(\sigma\left(\mathrm{R}_{\mathrm{E}}\right)\right)}{\longrightarrow} \mathrm{T}^{*} \mathrm{Q} \otimes \mathcal{W}^{\perp} \otimes \mathrm{S}_{2} \mathrm{~T}^{*} \mathrm{Q} \stackrel{\tau}{\longrightarrow} \Lambda_{2} \mathcal{W}^{\perp} \otimes \mathrm{S}_{2} \mathrm{~T}^{*} \mathrm{Q} \longrightarrow 0
$$

where $\tau$ is the canonical projection onto cokernel of $\rho_{1}\left(\sigma\left(\mathrm{R}_{\mathrm{E}}\right)\right)$.

Proof. Recall that

$$
\mathrm{S}_{2} \mathrm{~T}^{*} \mathrm{Q}=\mathrm{S}_{2} \mathcal{W} \oplus \mathrm{S}_{2} \mathcal{W}^{\perp} \oplus\left(\mathcal{W} \otimes \mathcal{W}^{\perp}\right)
$$

By using the definition of $\rho_{1}\left(\sigma\left(\mathrm{R}_{\mathrm{E}}\right)\right)$, the kernel of $\rho_{1}\left(\sigma\left(\mathrm{R}_{\mathrm{E}}\right)\right)$ is isomorphic to $\mathrm{S}_{2} \mathcal{W} \otimes$ $\mathrm{S}_{2} \mathrm{~T}^{*} \mathrm{Q}$. Moreover, we have

$$
\operatorname{Im}\left(\rho_{1}\left(\sigma\left(\mathrm{R}_{\mathrm{E}}\right)\right)\right) \cong\left(\mathrm{S}_{2} \mathcal{W}^{\perp} \oplus\left(\mathcal{W} \otimes \mathcal{W}^{\perp}\right)\right) \otimes \mathrm{S}_{2} \mathrm{~T}^{*} \mathrm{Q} .
$$

Therefore, the cokernel of $\rho_{1}\left(\sigma\left(\mathrm{R}_{\mathrm{E}}\right)\right)$ is isomorphic to $\Lambda_{2} \mathcal{W}^{\perp} \otimes \mathrm{S}_{2} \mathrm{~T}^{*} \mathrm{Q}$.

Proposition 6.4. The symbol of $\mathrm{R}_{\mathrm{E}}$ is involutive.

Proof. Let $\left\{e^{1}, \cdots, e^{n}\right\}$ be a basis for $\mathrm{T}_{q_{0}}^{*} \mathrm{Q}$ for $q_{0} \in \mathrm{Q}$ and let $\Sigma_{j}$ be the subspace of $\mathrm{T}_{q_{0}}^{*} \mathrm{Q}$ generated by $\left\{e^{j+1}, \cdots, e^{n}\right\}$. We show that this yields a quasi-linear basis for $\mathrm{T}_{q_{0}}^{*} \mathrm{Q} \mathrm{R}_{\mathrm{E}}$. Using Lemmata 6.1 and 6.2 we have

$$
\begin{aligned}
\operatorname{dim}\left(\mathrm{G}\left(\mathrm{R}_{\mathrm{E}}\right)\right) & =\frac{1}{2}(n-m) n(n+1), \\
\operatorname{dim}\left(\mathrm{G}\left(\mathrm{R}_{\mathrm{E}}\right)_{1, j}\right) & =\left\{\begin{array}{cl}
\frac{1}{2}(n-m-j) n(n+1), & 1 \leq j<n-m, \\
0, & n-m \leq j<0 .
\end{array}\right.
\end{aligned}
$$

We compute

$$
\operatorname{dim}\left(\mathrm{G}\left(\mathrm{R}_{\mathrm{E}}\right)\right)+\sum_{j=1}^{n-m} \operatorname{dim}\left(\mathrm{G}\left(\mathrm{R}_{\mathrm{E}}\right)_{1, j}\right)=\frac{1}{4} n(n-m)(n-m+1)(n+1),
$$

which is equal to $\operatorname{dim}\left(\rho_{1}\left(\mathrm{G}\left(\mathrm{R}_{\mathrm{E}}\right)\right)\right)$ by Lemma 6.3 , as required.

6.2. Involutivity of $R_{\mathbf{E}}$. The following theorem applies Goldschmidt's theorem to $\mathrm{R}_{\mathrm{E}}$.

THEOREM 6.5. The partial differential equation $\mathrm{R}_{\mathrm{E}}$ is involutive if, for $p \in \mathrm{R}_{\mathrm{E}}$, we have

$$
\tau\left(\rho_{1}\left(p_{2}\right)-0\right)=0,
$$

where $p_{2}$ is any point in $\mathrm{J}_{2}(\pi)$ that projects to $p$.

Proof. Since the symbol is involutive, the proof follows along the same lines as that of Theorem 5.5. 
One can construct the curvature map similar to the one for $\mathrm{R}_{\mathrm{L}}$ :

$$
\kappa\left(j_{1} \mathbb{G}_{\mathrm{cl}}\right)(\lambda P X, \lambda P Y, Z, Z)=
$$

$(28) \nabla_{\lambda P Y}^{\mathbb{G}}\left[\nabla_{\lambda P X}^{\mathbb{G}}\left(\mathbb{G}_{\mathrm{cl}}\right)(Z, Z)\right]-\nabla_{\lambda P X}^{\mathbb{G}}\left[\nabla_{\lambda P Y}^{\mathbb{G}}\left(\mathbb{G}_{\mathrm{cl}}\right)(Z, Z)\right]-\nabla_{[\lambda P Y, \lambda P X]}^{\mathbb{G}}\left(\mathbb{G}_{\mathrm{cl}}\right)(Z, Z)$.

Note that since $\lambda\left(\mathrm{TQ} /\left(\mathbb{G}_{\mathrm{ol}}^{\sharp} \mathcal{W}\right)\right)$ is integrable by assumption, there exists a $\zeta \in \Gamma^{\omega}(\mathrm{TQ})$ such that $[\lambda P Y, \lambda P X]=\lambda P \zeta$. We state the following theorem which implies the obstruction to finding a closed-loop metric.

THEOREM 6.6. Let $(\mathrm{Q}, \mathbb{G})$ be an analytic Riemannian manifold of dimension $n$ and let $\mathcal{S}$ be the Levi-Civita connection on $\mathrm{Q}$ with associated curvature tensor $\mathrm{R}$. Let $\mathcal{W} \subset \mathrm{T}^{*} \mathrm{Q}$ be an analytic integrable subbundle and let $P \in \Gamma^{\omega}\left(\mathrm{T}^{*} \mathrm{Q} \otimes \mathrm{TQ}\right)$ be the associated $\mathbb{G}$-orthogonal projection as above. Let $\lambda$ be an automorphism on $\mathrm{TQ}$ so that $\lambda\left(\mathrm{TQ} /\left(\mathbb{G}^{\sharp} \mathcal{W}\right)\right)$ is integrable. If the first-order partial differential equations

$$
\begin{aligned}
2 \nabla_{\lambda P Y}^{\mathbb{G}}\left(\mathbb{G}_{\mathrm{cl}}\right)( & {[Z, \lambda P X], Z)-2 \nabla_{\lambda P X}^{\mathbb{G}}\left(\mathbb{G}_{\mathrm{cl}}\right)([Z, \lambda P Y], Z) } \\
+ & 2 \mathbb{G}_{\mathrm{cl}}\left(\nabla_{\lambda P Y}^{\mathbb{G}}\left(\nabla_{Z}^{\mathbb{G}} \lambda P X\right)-\nabla_{\lambda P X}^{\mathbb{G}}\left(\nabla_{Z}^{\mathbb{G}} \lambda P Y\right), Z\right) \\
& +2 \mathbb{G}\left(\nabla_{\lambda P Y}^{\mathbb{G}}\left(\nabla_{Z}^{\mathbb{G}} P X\right)-\nabla_{\lambda P X}^{\mathbb{G}}\left(\nabla_{Z}^{\mathbb{G}} P Y\right), Z\right) \\
+2 \mathbb{G}_{\mathrm{cl}}\left(\nabla_{\lambda P Y}^{\mathbb{G}} Z,\right. & \left.\nabla_{Z}^{\mathbb{G}} \lambda P X\right)-2 \mathbb{G}_{\mathrm{cl}}\left(\nabla_{\lambda P X}^{\mathbb{G}} Z, \nabla_{Z}^{\mathbb{G}} \lambda P Y\right)+2 \mathbb{G}\left(\nabla_{\lambda P X}^{\mathbb{G}} Z, \nabla_{Z}^{\mathbb{G}} \lambda P Y\right) \\
& -2 \mathbb{G}_{\mathrm{cl}}\left(\nabla_{\lambda P Y}^{\mathbb{G}} Z, \nabla_{Z}^{\mathbb{G}} \lambda P X\right)+2 \mathbb{G}_{\mathrm{cl}}\left(\nabla_{Z}^{\mathbb{G}} \lambda \zeta, Z\right)-2 \mathbb{G}\left(\nabla_{Z}^{\mathbb{G}} \zeta, Z\right)=0
\end{aligned}
$$

are satisfied in a neighborhood of $\mathbb{G}_{\mathrm{cl}} \in \Gamma^{\omega}\left(\mathrm{S}_{2} \mathrm{~T}^{*} \mathrm{Q}\right)$ for $X, Y, Z \in \Gamma^{\omega}(\mathrm{TQ})$ and $\zeta \in$ $\Gamma^{\omega}(\mathrm{TQ})$ as above, then the set of closed-loop metric equations has a solution in a neighborhood of $\mathbb{G}_{\mathrm{cl}}$. Moreover, any solution to the closed-loop metric equations will satisfy equation (29).

Proof. The proof follows from a direct computation using equation (28) and the Ricci identity (similar to Theorem 5.6). One also uses the following identity

$$
\begin{aligned}
\left(\mathbb{G}_{\mathrm{cl}}\right)(\mathrm{R}(Z, Z) \lambda P Y, \lambda P X)+\left(\mathbb{G}_{\mathrm{cl}}\right)(\lambda P Y, \mathrm{R}(Z, Z) \lambda P X) & = \\
(\mathbb{G})(\mathrm{R}(Z, Z) \lambda P Y, P X)+(\mathbb{G})(P Y, \mathrm{R}(Z, Z) \lambda P X) & =0,
\end{aligned}
$$

which holds since $\mathbb{G}$ is associated to $\mathcal{S}$.

7. Formal integrability of $R_{\mathbf{T}}$. We prove that the system of partial differential equations relating the $\lambda$-equations to the potential energy shaping equations is formally integrable under a surjectivity condition. The main result here is Theorem 7.8. As with the previous two sections, this result can be understood separately from the details of its proof.

7.1. The symbol of $R_{T}$. The symbol map for $R_{T}$ can be written precisely as the following morphism of vector bundles:

$$
\sigma\left(\mathrm{R}_{\mathrm{T}}\right): \pi_{1}^{*} \mathrm{~T}^{*} \mathrm{Q} \otimes\left(\pi_{0}^{1}\right)^{*} \mathrm{~V} \pi \rightarrow \mathrm{V} \pi^{\prime}
$$


where $\pi^{\prime}$ denotes the bundle $\left(\pi^{\prime}, \Lambda_{2} \mathcal{W}^{\perp}, \mathrm{Q}\right)$. If we evaluate the morphism $\sigma$ at a given point $p$, we obtain the following exact sequence, which characterizes the symbol, $\mathrm{G}\left(\mathrm{R}_{\mathrm{T}}\right)$, at $p$ :

$$
\left.0 \longrightarrow \mathrm{G}\left(\mathrm{R}_{\mathrm{T}}\right)\right|_{p} \longrightarrow \mathrm{T}_{\pi_{1}(p)}^{*} \mathrm{Q} \otimes \mathrm{V}_{\pi_{0}^{1}(p)}(\pi) \stackrel{\sigma(\Phi)}{\longrightarrow} \mathrm{V}_{\Phi(p)} \pi^{\prime}
$$

Note that the linearization $\mathrm{V} \Phi$ about a point $p \in \mathrm{J}_{1} \pi$ with

$$
\pi_{0}^{1}(p)=\left(\left.\left.\mathrm{id}\right|_{\mathrm{TQ} \otimes Q_{\mathcal{Q}} \mathcal{W} \perp} \otimes \mathrm{id}\right|_{\mathrm{TQ} \otimes_{\mathrm{Q}} \mathcal{W}^{\perp}}\right),
$$

can be reduced to a map on $\left.J_{1} \vee \pi\right|_{\left(\mathcal{W}^{\perp} \otimes_{\mathrm{Q}} \mathrm{TQ}\right) \oplus 0}$ since there is no $\Delta$ involved in the linearization (see equation (22)). If we identify $\bigvee_{\pi_{0}^{k}(p)}(\pi)$ and $\bigvee_{\Phi(p)}\left(\pi^{\prime}\right)$ with $\left(\mathcal{W}^{\perp} \otimes_{\mathbf{Q}}\right.$ $\mathrm{TQ})\left.\right|_{\pi_{0}^{1}(p)}$ and $\left.\Lambda_{2}\left(\mathcal{W}^{\perp}\right)\right|_{\Phi(p)}$, respectively, we can write the symbol map as

$$
\left.\sigma\left(\mathrm{R}_{\mathrm{T}}\right)\right|_{p}:\left.\left.\mathrm{T}_{\pi_{1}(p)}^{*} \mathrm{Q} \otimes\left(\mathcal{W}^{\perp} \otimes_{\mathrm{Q}} \mathrm{TQ}\right)\right|_{\pi_{0}^{1}(p)} \rightarrow \Lambda_{2}\left(\mathcal{W}^{\perp}\right)\right|_{\Phi(p)}
$$

We usually drop the points of evaluation for simplicity of notation. Consider the alternation map Alt acting on the (0,2)-tensors and denote the restriction of 2Alt to $\left.\left(\mathcal{W}^{\perp} \otimes \mathrm{T}^{*} \mathrm{Q}\right)\right|_{\pi_{0}^{1}(p)}$ by $\check{\sigma}$. Explicitly, if we have $\mathrm{b} \in \mathcal{W}_{\pi_{1}(p)}^{\perp}$ and $c \in \mathrm{T}_{\pi_{1}(p)}^{*} \mathrm{Q}$, then

$$
\check{\sigma}(\mathrm{b} \otimes \mathrm{c})\left(u_{1} \oplus u_{2}, v_{1} \oplus v_{2}\right)=\mathrm{b}\left(u_{1}\right) \mathrm{c}\left(v_{1} \oplus v_{2}\right)-\mathrm{b}\left(v_{1}\right) \mathrm{c}\left(u_{1} \oplus u_{2}\right) .
$$

Lemma 7.1. We have $\operatorname{ker}(\check{\sigma})=\mathrm{S}_{2} \mathcal{W}^{\perp}$ and $\operatorname{Im}(\check{\sigma})=\mathrm{I}_{2}\left(\mathcal{W}^{\perp}\right)$.

Proof. Since $\check{\sigma}$ is the restriction of the alternation map, one can easily observe that $\operatorname{ker}(\check{\sigma})=\operatorname{ker}($ Alt $) \cap\left(\mathcal{W}^{\perp} \otimes \mathrm{T}^{*} \mathrm{Q}\right)$. Clearly $\mathrm{S}_{2} \mathcal{W}^{\perp} \subset \mathrm{S}_{2} \mathrm{~T}^{*} \mathrm{Q} \cap\left(\mathcal{W}^{\perp} \otimes \mathrm{T}^{*} \mathrm{Q}\right)$. Moreover, for any $\Theta \in \Gamma^{\omega}\left(S_{2} T^{*} Q \cap\left(\mathcal{W}^{\perp} \otimes T^{*} Q\right)\right)$, we have

$$
\Theta\left(u_{1} \oplus u_{2}, v_{1} \oplus v_{2}\right)=\Theta\left(0 \oplus v_{2}, u_{1} \oplus u_{2}\right)=\Theta\left(0 \oplus u_{2}, 0 \oplus v_{2}\right) .
$$

Thus $\Theta \in \Gamma^{\omega}\left(S_{2} \mathcal{W}^{\perp}\right)$ and as a result we have $S_{2} T^{*} Q \cap\left(\mathcal{W}^{\perp} \otimes T^{*} Q\right)=S_{2} \mathcal{W}^{\perp}$. Recalling that $\Lambda_{2}\left(\mathrm{~T}^{*} \mathrm{Q}\right)=\Lambda_{2}(\mathcal{W}) \oplus \Lambda_{2}\left(\mathcal{W}^{\perp}\right) \oplus\left(\mathcal{W} \otimes \mathcal{W}^{\perp}\right)$ and using the definition of $\check{\sigma}$, one can observe that $\operatorname{Im}(2 \mathrm{Alt})=\operatorname{Im}(\check{\sigma}) \cup \Lambda_{2}(\mathcal{W})$.

The symbol map $\left.\sigma\left(\mathrm{R}_{\mathrm{T}}\right)\right|_{p}$ can be characterized as the following composition map:

$$
\mathrm{T}^{*} \mathrm{Q} \otimes\left(\mathcal{W}^{\perp} \otimes_{\mathrm{Q}} \mathrm{TQ}\right) \stackrel{\tilde{\sigma}}{\longrightarrow} \mathrm{I}_{2}\left(\mathcal{W}^{\perp}\right) \stackrel{\mathrm{p}}{\longrightarrow} \Lambda_{2} \mathcal{W}^{\perp}
$$

where $\mathrm{p}$ is the canonical projection of $\mathrm{I}_{2}\left(\mathcal{W}^{\perp}\right)$ onto $\Lambda_{2} \mathcal{W}^{\perp}$ and

$$
\tilde{\sigma}(\mathbf{b} \otimes \mathbf{c} \otimes v)=\beta(v) \check{\sigma}(\mathbf{b} \otimes \mathbf{c}),
$$

with $\beta \in \mathrm{T}_{\pi_{1}(p)}^{*} \mathrm{Q}, \mathrm{b} \in \mathcal{W}_{\pi_{1}(p)}^{\perp}, \mathrm{c} \in \mathrm{T}_{\pi_{1}(p)}^{*} \mathrm{Q}$ and $v \in \mathrm{T}_{\pi_{1}(p)} \mathrm{Q}$.

LEMMA 7.2. The following sequence is short exact:

$$
0 \longrightarrow \mathrm{G}\left(\mathrm{R}_{\mathrm{T}}\right) \longrightarrow \mathrm{T}^{*} \mathrm{Q} \otimes\left(\mathcal{W}^{\perp} \otimes_{\mathrm{Q}} \mathrm{TQ}\right)^{\sigma\left(\mathrm{R}_{\mathrm{T}}\right)} \longrightarrow \Lambda_{2}\left(\mathcal{W}^{\perp}\right)
$$


where

$$
\mathrm{G}\left(\mathrm{R}_{\mathrm{T}}\right) \cong\left(\mathrm{S}_{2} \mathcal{W}^{\perp} \otimes \mathrm{TQ}\right) \oplus\left(\left(\mathcal{W} \otimes \mathcal{W}^{\perp}\right) \otimes \mathrm{TQ}\right) \oplus\left(\Lambda_{2} \mathcal{W}^{\perp} \otimes \operatorname{ann}(\beta)\right) .
$$

Proof. Clearly $\mathrm{T}^{*} \mathrm{Q} \otimes\left(\mathcal{W}^{\perp} \otimes \operatorname{ann}(\beta)\right) \subset \operatorname{ker}\left(\sigma\left(\mathrm{R}_{\mathrm{T}}\right)\right)$. Lemma 7.2 yields $\mathrm{S}_{2} \mathcal{W}^{\perp} \otimes$ $\mathrm{TQ} \subset \operatorname{ker} \sigma\left(\mathrm{R}_{\mathrm{T}}\right)$. Finally $\tilde{\sigma}\left(\left(\mathcal{W} \otimes \mathcal{W}^{\perp}\right) \otimes \mathrm{TQ}\right) \subset$ ker $\mathrm{p}$. The claim follows since the image of $\Lambda_{2} \mathcal{W}^{\perp} \otimes v$ under $\sigma\left(\mathrm{R}_{\mathrm{T}}\right)$ is $\Lambda_{2} \mathcal{W}^{\perp}$, where $v \in \mathrm{T}_{\pi_{1}(p)} \mathrm{Q} / \operatorname{ann}(\beta)$.

Let $\left\{e^{1}, \cdots, e^{n}\right\}$ be a basis for $\mathrm{T}_{\pi_{1}(p)}^{*} \mathrm{Q}$. Let $\Sigma_{j}$ be the subspace of $\mathrm{T}_{\pi_{1}(p)}^{*} \mathrm{Q}$ generated by $\left\{e^{j+1}, \cdots, e^{n}\right\}$ and define $\mathrm{M}^{*}{ }_{j}=\mathcal{W}^{\perp} \cap \Sigma_{j}$ and $\mathrm{M}^{*}{ }_{j}^{\perp}=\mathcal{W}^{\perp} \cap \Sigma_{j}^{\perp}$. Let $\mathrm{I}_{2}\left(\mathrm{M}^{*}{ }_{j}\right)=$ $\mathrm{I}\left(\mathrm{M}^{*}{ }_{j}\right) \cap \Lambda_{2}\left(\mathcal{W}^{\perp}\right)$. The following two lemmata can be proved along the same lines as Lemma 7.2.

Lemma 7.3. The following sequence is short exact:

$$
0 \longrightarrow \mathrm{G}\left(\mathrm{R}_{\mathrm{T}}\right)_{1, j} \longrightarrow \Sigma_{j} \otimes\left(\mathcal{W}^{\perp} \otimes_{\mathrm{Q}} \mathrm{TQ}\right) \stackrel{\sigma\left(\mathrm{R}_{\mathrm{T}}\right)}{\longrightarrow} \mathrm{I}_{2}\left(\mathrm{M}^{*}{ }_{j}\right)
$$

where

$$
\begin{aligned}
\mathrm{G}\left(\mathrm{R}_{\mathrm{T}}\right)_{1, j} \cong\left(\mathrm{S}_{2} \mathrm{M}_{\mathrm{j}}^{*} \otimes \mathrm{TQ}\right) & \oplus\left(\left(\left(\mathcal{W} \cap \Sigma_{j}\right) \otimes \mathcal{W}^{\perp}\right) \otimes \mathrm{TQ}\right) \oplus \\
& \left(\left(\left(\mathcal{W}^{\perp} \cap \Sigma_{j}\right) \otimes \mathcal{W}^{\perp}\right) / \mathrm{S}_{2} \mathrm{M}_{\mathrm{j}}^{*} \otimes \operatorname{ann}(\beta)\right)
\end{aligned}
$$

One can identify the prolongation map $\rho_{1}\left(\sigma\left(\mathrm{R}_{\mathrm{T}}\right)\right)$ as

$$
\rho_{1}\left(\sigma\left(\mathrm{R}_{\mathrm{T}}\right)\right)(\mathrm{c} \cdot \mathrm{d} \otimes \mathrm{b} \otimes v)=\frac{1}{2} \beta(v)(\mathbf{c} \otimes \check{\sigma}(\mathbf{d} \otimes \mathbf{b})+\mathrm{d} \otimes \check{\sigma}(\mathbf{c} \otimes \mathbf{b})),
$$

where $\beta \in \mathrm{T}_{\pi_{1}(p)}^{*} \mathrm{Q}, \mathrm{b} \in \mathcal{W}_{\pi_{1}(p)}^{\perp}, \mathrm{c}, \mathrm{d} \in \mathrm{T}_{\pi_{1}(p)}^{*} \mathrm{Q}$ and $v \in \mathrm{T}_{\pi_{1}(p)} \mathrm{Q}$.

LEMMA 7.4. The following sequence is short exact:

$$
0 \longrightarrow \rho_{1}\left(\mathrm{G}\left(\mathrm{R}_{\mathrm{T}}\right)\right) \longrightarrow \mathrm{S}_{2} \mathrm{~T}^{*} \mathrm{Q} \otimes\left(\mathcal{W}^{\perp} \otimes_{\mathrm{Q}} \mathrm{TQ}\right)^{\rho_{1}\left(\sigma\left(\mathrm{R}_{\mathrm{T}}\right)\right)} \longrightarrow \mathrm{T}^{*} \mathrm{Q} \otimes \Lambda_{2} \mathcal{W}^{\perp}
$$

where

$$
\begin{aligned}
\rho_{1}\left(\mathrm{G}\left(\mathrm{R}_{\mathrm{T}}\right)\right) \cong & \left(\mathrm{S}_{3} \mathcal{W}^{\perp} \otimes \mathrm{TQ}\right) \oplus\left(\left(\mathcal{W} \otimes \mathcal{W}^{\perp} \otimes \mathcal{W}^{\perp}\right) \otimes \mathrm{TQ}\right) \\
& \oplus\left(\left(\mathrm{S}_{2} \mathcal{W} \otimes \mathcal{W}^{\perp}\right) \otimes \mathrm{TQ}\right) \oplus\left(\left(\left(\mathrm{S}_{2} \mathcal{W}^{\perp} \otimes \mathcal{W}^{\perp}\right) / \mathrm{S}_{3} \mathcal{W}^{\perp}\right) \otimes \operatorname{ann}(\beta)\right) .
\end{aligned}
$$

Proposition 7.5. The symbol of $\mathrm{R}_{\mathrm{T}}$ is involutive.

Proof. We will show that the basis $\left\{e^{1}, \cdots, e^{n}\right\}$ above is a quasi-regular basis. Using Lemmata 7.2 and 7.3 we have

$$
\begin{aligned}
\operatorname{dim}\left(\mathrm{G}\left(\mathrm{R}_{\mathrm{T}}\right)\right)= & \frac{m(m+1)}{2} n+m n(n-m)+\frac{m(m-1)}{2}(n-1), \\
\operatorname{dim}\left(\mathrm{G}\left(\mathrm{R}_{\mathrm{T}}\right)_{1, j}\right)= & n \frac{(m-j+1)(m-j)}{2}+m n(n-m) \\
& +\left[(m-j) m-\frac{(m-j)(m-j+1)}{2}\right](n-1), \quad j<m, \\
\operatorname{dim}\left(\mathrm{G}\left(\mathrm{R}_{\mathrm{T}}\right)_{1, j}\right)= & m n(n-j), \quad j \geq m .
\end{aligned}
$$


As a result we compute

$$
\begin{aligned}
\sum_{j=1}^{n-1} \operatorname{dim}\left(\mathrm{G}\left(\mathrm{R}_{\mathrm{T}}\right)_{1, j}\right)+\operatorname{dim}\left(\mathrm{G}\left(\mathrm{R}_{\mathrm{T}}\right)\right)= & \sum_{j=1}^{m-1}\left(n \frac{(m-j+1)(m-j)}{2}+m n(n-m)\right. \\
& \left.+\left[(m-j) m-\frac{(m-j)(m-j+1)}{2}\right](n-1)\right) \\
& +\sum_{j=m}^{n-1}(m n(n-j))+n \frac{m(m+1)}{2} \\
& +m n(n-m)+\frac{m(m-1)}{2}(n-1) \\
= & \frac{1}{6} m n(m+1)(m+2)+\frac{1}{2} m n(n+m+1)(n-m) \\
& +\frac{1}{3} m(m-1)(m+1)(n-1),
\end{aligned}
$$

which is precisely dimension of $\operatorname{dim}\left(\rho_{1}\left(\mathrm{G}\left(\mathrm{R}_{\mathrm{T}}\right)\right)\right)$ by Lemma 7.4.

7.2. Involutivity of $R_{T}$. To compute the curvature map for $R_{T}$ we use the following lemma.

LEMma 7.6. The following sequence is exact:

$$
\mathrm{S}_{2} \mathrm{~T}^{*} \mathrm{Q} \otimes\left(\mathcal{W}^{\perp} \otimes \mathrm{Q} T \mathrm{Q}\right)^{Q_{1}\left(\sigma\left(\mathrm{R}_{\mathrm{T}}\right)\right)} \longrightarrow \mathrm{T}^{*} \mathrm{Q} \otimes \Lambda_{2} \mathcal{W}^{\perp} \stackrel{\tau}{\longrightarrow} \Lambda_{3} \mathcal{W}^{\perp} \oplus\left(\mathcal{W} \otimes \Lambda_{2} \mathcal{W}^{\perp}\right) \longrightarrow 0
$$

where $\tau$ is the projection to the $\operatorname{coker}\left(\rho_{1}\left(\sigma\left(\mathrm{R}_{\mathrm{T}}\right)\right)\right)$ given by

$$
\begin{aligned}
& \tau(\mathrm{b})\left(v_{1} \oplus v_{2}, u, w\right)= \\
& \left(\mathrm{b}\left(v_{1}, u, w\right)+\mathrm{b}\left(u, w, v_{1}\right)+\mathrm{b}\left(w, v_{1}, u\right)\right)+\mathrm{b}\left(v_{2}, u, w\right), \quad v_{1}, u, w \in \mathcal{W}^{\perp}, v_{2} \in \mathcal{W} .
\end{aligned}
$$

Proof. Recall that $\mathrm{S}_{2} \mathrm{~T}^{*} \mathrm{Q}=\mathrm{S}_{2} \mathcal{W} \oplus \mathrm{S}_{2} \mathcal{W}^{\perp} \oplus\left(\mathcal{W} \otimes \mathcal{W}^{\perp}\right)$. Using Lemma 7.4 and since

$$
\left(\mathrm{S}_{3} \mathcal{W}^{\perp} \otimes \mathrm{TQ}\right) \oplus\left(\left(\mathcal{W} \otimes \mathcal{W}^{\perp} \otimes \mathcal{W}^{\perp}\right) \otimes \mathrm{TQ}\right) \subseteq \operatorname{ker}\left(\rho_{1}\left(\sigma\left(\mathrm{R}_{\mathrm{T}}\right)\right)\right)
$$

we observe that

$$
\mathcal{W} \otimes \Lambda_{2} \mathcal{W}^{\perp} \subseteq \operatorname{coker}\left(\rho_{1}\left(\sigma\left(\mathrm{R}_{\mathrm{T}}\right)\right)\right)
$$

Moreover, by definition the image of $\rho_{1}\left(\sigma\left(\mathrm{R}_{\mathrm{T}}\right)\right)$ is symmetric in the first two elements, so one can conclude that

$$
\Lambda_{3} \mathcal{W}^{\perp} \subseteq \operatorname{coker}\left(\rho_{1}\left(\sigma\left(\mathrm{R}_{\mathrm{T}}\right)\right)\right)
$$

Finally, a direct computation shows that, for any $\xi \in \Gamma^{\omega}\left(T^{*} Q \otimes \Lambda_{2} \mathcal{W}^{\perp}\right)$, there exists an analytic section of $\mathrm{T}^{*} \mathrm{Q} \otimes \Lambda_{2} \mathcal{W}^{\perp}$ which projects to $\xi$ under $\tau$.

As a consequence of the previous computations, we have the following theorem. 
THEOREM 7.7. The partial differential equation $\mathrm{R}_{\mathrm{T}}$ is involutive if, for $p \in \mathrm{R}_{\mathrm{T}}$, we have

$$
\tau\left(\rho_{1}(\Phi)\left(p_{2}\right)-0\right)=0
$$

where $p_{2}$ is any point in $\mathrm{J}_{2} \pi$ that projects to $p$.

Proof. The proof follows similarly to Theorem 5.5. Notice that $\rho_{1}\left(\mathrm{G}_{1}\left(\mathrm{R}_{\mathrm{L}}\right)\right)$ is a vector bundle on the open subset on which $\mathcal{W}$ is a vector bundle. Since $G\left(R_{T}\right)$ is an involutive symbol, the system of partial differential equations $R_{T}$ is involutive if the curvature map $\kappa$ defined as following is zero:

$$
\kappa: \mathrm{R}_{\mathrm{T}} \rightarrow \Lambda_{3} \mathcal{W}^{\perp} \oplus\left(\mathcal{W} \otimes \Lambda_{2} \mathcal{W}^{\perp}\right)
$$

with $\kappa(p)=\tau\left(\rho_{1}(\Phi)\left(p_{2}\right)\right)$, where $p_{2}$ is any point in $\mathrm{J}_{2}(\pi)$ that projects to $p$.

Consider the bundle $\pi^{\prime}: \Lambda_{2} \mathcal{W}^{\perp} \rightarrow \mathrm{Q}$ and let $p \in \mathrm{R}_{\mathrm{T}}$ be such that $\pi(p)=q$ for $q \in \mathrm{Q}$. Therefore, $\Phi(p)=0$. Take $p_{2} \in \mathrm{J}_{2}(\pi)$ projecting to $p$ and define $\xi=$ $\rho_{1}(\Phi)\left(p_{2}\right) \in J_{1}\left(\pi^{\prime}\right)$. One can show that $\xi$ projects to $0 \in \Gamma^{\omega}\left(\Lambda_{2} \mathcal{W}^{\perp}\right)$, so we take $\kappa(p)=\tau(\xi-0)$. It is easy to show that the definition of $\kappa$ is independent of the choice of $p_{2}[27,28]$. By the discussion we had in Section 3.1, $p$ is in the projection of $\rho_{1}\left(\mathrm{R}_{\mathrm{T}}\right)$ to $\mathrm{R}_{\mathrm{T}}$ if and only if $\kappa(p)=0$.

Recall the definition of $\mathrm{R}_{\mathrm{pot}}$ from Section 4. We have the following theorem.

TheOrem 7.8. Let $\Sigma_{\mathrm{ol}}=\left(\mathrm{Q}, \mathbb{G}_{\mathrm{ol}}, V_{\mathrm{ol}}, \mathcal{F}_{\mathrm{ol}}, \mathcal{W}_{\mathrm{ol}}\right)$ be an analytic open-loop simple mechanical control system. Let $p_{0} \in \mathrm{R}_{\mathrm{pot}}$ and let $q_{0}=\pi_{1}\left(p_{0}\right)$. Assume that $q_{0}$ is a regular point for $\mathcal{W}_{\mathrm{ol}}$ and that there exists a bundle automorphism $\Theta$ on $\mathrm{T}^{*} \mathrm{Q}$ defined on a neighborhood $U$ of $q_{0}$ such that $\Theta$ satisfies the following equation in the neighborhood $U$ :

$$
\begin{aligned}
{[\kappa(\Theta)]_{r i j}=} & \left(\frac{\partial^{2} \Theta_{i}^{l}}{\partial q^{r} \partial q^{j}} \frac{\partial V_{\mathrm{ol}}}{\partial q^{l}}-\frac{\partial^{2} \Theta_{j}^{l}}{\partial q^{r} \partial q^{i}} \frac{\partial V_{\mathrm{ol}}}{\partial q^{l}}\right)+\left(\frac{\partial \Theta_{i}^{l}}{\partial q^{j}} \frac{\partial^{2} V_{\mathrm{ol}}}{\partial q^{r} \partial q^{l}}-\frac{\partial \Theta_{j}^{l}}{\partial q^{i}} \frac{\partial^{2} V_{\mathrm{ol}}}{\partial q^{r} \partial q^{l}}\right) \\
& +\left(\frac{\partial \Theta_{i}^{l}}{\partial q^{r}} \frac{\partial^{2} V_{\mathrm{ol}}}{\partial q^{j} \partial q^{l}}-\frac{\partial \Theta_{j}^{l}}{\partial q^{r}} \frac{\partial^{2} V_{\mathrm{ol}}}{\partial q^{i} \partial q^{l}}\right)+\left(\Theta_{i}^{l} \frac{\partial^{3} V_{\mathrm{ol}}}{\partial q^{r} \partial q^{j} \partial q^{l}}-\Theta_{j}^{l} \frac{\partial^{3} V_{\mathrm{ol}}}{\partial q^{r} \partial q^{i} \partial q^{l}}\right)=0,
\end{aligned}
$$

where $i, j \in\{1, \cdots, m\}, l \in\{1, \cdots, n\}$ and $r \in\{m+1, \cdots, n\}$. Then there exists an analytic closed-loop energy shaping metric $\mathbb{G}_{\mathrm{cl}}$ prescribed by $\mathbb{G}_{\mathrm{cl}}^{b}=\Theta \circ \mathbb{G}_{\mathrm{ol}}^{b}$ and an analytic $\left(\mathbb{G}_{\mathrm{ol}}-\mathbb{G}_{\mathrm{cl}}\right)$-potential energy shaping feedback $\mathcal{F} \in \Gamma^{\omega}\left(\mathcal{W}_{\mathrm{ol}}\right)$ defined on $U$ which satisfies $\Phi_{\mathrm{pot}}\left(p_{0}\right)=\Theta^{-1} d V\left(q_{0}\right)-d V_{\mathrm{ol}}\left(q_{0}\right)+\Theta d V_{\mathrm{ol}}\left(q_{0}\right)$ for a solution $V$ to $\mathrm{R}_{\mathrm{pot}}$.

Proof. Observe that the system of partial differential equations $\mathrm{R}_{\mathrm{T}}$, with $\Theta=$ $\Lambda_{\mathrm{cl}}^{-1}$ and $\beta=d V_{\mathrm{ol}}$, prescribes the sufficient conditions for existence of a $\left(\mathbb{G}_{\mathrm{ol}}-\mathbb{G}_{\mathrm{cl}}\right)$ potential energy shaping feedback; see equation (21). Using Theorem 7.7, this partial differential equation is integrable if the curvature map given by equation (33) is zero. 
A direct computation shows that the curvature map is given in local coordinates by:

$$
\begin{aligned}
{[\kappa(\Theta)]=} & \left(\frac{\partial^{2} \Theta_{i}^{l}}{\partial q^{r} \partial q^{j}} \frac{\partial V_{\mathrm{ol}}}{\partial q^{l}}-\frac{\partial^{2} \Theta_{j}^{l}}{\partial q^{r} \partial q^{i}} \frac{\partial V_{\mathrm{ol}}}{\partial q^{l}}+\frac{\partial \Theta_{i}^{l}}{\partial q^{j}} \frac{\partial^{2} V_{\mathrm{ol}}}{\partial q^{r} \partial q^{l}}-\frac{\partial \Theta_{j}^{l}}{\partial q^{i}} \frac{\partial^{2} V_{\mathrm{ol}}}{\partial q^{r} \partial q^{l}}\right. \\
& \left.+\frac{\partial \Theta_{i}^{l}}{\partial q^{r}} \frac{\partial^{2} V_{\mathrm{ol}}}{\partial q^{j} \partial q^{l}}-\frac{\partial \Theta_{j}^{l}}{\partial q^{r}} \frac{\partial^{2} V_{\mathrm{ol}}}{\partial q^{i} \partial q^{l}}+\Theta_{i}^{l} \frac{\partial^{3} V_{\mathrm{ol}}}{\partial q^{r} \partial q^{j} \partial q^{l}}-\Theta_{j}^{l} \frac{\partial^{3} V_{\mathrm{ol}}}{\partial q^{r} \partial q^{i} \partial q^{l}}\right) d q^{r} \\
& \otimes d q^{i} \wedge d q^{j},
\end{aligned}
$$

where $i, j \in\{1, \cdots, m\}, l \in\{1, \cdots, n\}$ and $r \in\{m+1, \cdots, n\}$, as desired.

As an example, we discuss systems with one degree of underactuation.

EXAMPLE 7.9 (Systems with one degree of underactuation). Numerous systems considered in the literature on energy shaping have one degree of underactuation. In [4], the authors introduce a coordinate system that transforms the system of partial differential equations into a homogenous equation. Using the results of this section, one can give a complete description of the system of partial differential equations with one degree of underactuation. The following theorem shows that in this case it is enough to find the set of bundle automorphisms which satisfy the sufficient conditions of Theorem 5.6.

THEOREM 7.10. If $\Sigma_{\mathrm{ol}}$ is a simple mechanical control system with one degree of underactuation, for each bundle automorphism which satisfies the set of $\lambda$-equations, there exists a closed-loop metric and a closed-loop potential function which satisfies the energy shaping system of partial differential equations.

Proof. Note that the projection map $\tau$ in Lemma 7.6 is a zero map for $m=$ 1 and so the closed-loop metric equation is involutive by Theorem 6.6. Moreover, equation (21) always holds for $m=1$.

8. Summary. In this section we give a summary of the theorems we have obtained in the previous sections. Moreover, we state a procedure that clarifies how one should perform the energy shaping method so that certain problems - such as having a closed-loop energy shaping metric for which no potential energy shaping is possible - will not happen. This procedure reveals some of the fundamental properties of energy shaping partial differential equations that have not been addressed before.

1. Kinetic energy shaping: Find the set of bundle automorphisms $\lambda$ on TQ which satisfy the sufficient conditions of Theorem 5.6 and denote it by $\hat{S}_{K}$. Use the sufficient conditions of Theorem 6.6 to find the set of $\lambda \in \hat{S}_{K}$ for which there exists a closed-loop energy shaping metric $\mathbb{G}_{\mathrm{cl}}$ and denote it by $\mathrm{S}_{\mathrm{K}}$.

2. Potential energy shaping: Find the set of bundle automorphisms $\Theta$ on $\mathrm{T}^{*} \mathrm{Q}$ which satisfy the sufficient conditions of Theorem 7.8 and denote it by $\hat{S}_{\mathrm{P}}$ and let

$$
\mathrm{S}_{\mathrm{P}}^{\prime}=\left\{\Theta^{-1} \mid \Theta \in \hat{\mathrm{S}}_{\mathrm{P}}\right\}
$$


The set of bundle automorphisms $S_{P}^{\prime}$ induces a set of bundle automorphisms on $T Q$ by

$$
\mathrm{S}_{\mathrm{P}} \doteq\left\{\mathbb{G}_{\mathrm{ol}}^{\sharp} \Lambda \mathbb{G}_{\mathrm{ol}}^{b} \mid \Lambda \in \mathrm{S}_{\mathrm{P}}^{\prime}\right\}
$$

Note that by Theorem 4.10, for each $\lambda \in \mathrm{S}_{\mathrm{P}}$ there exists a $V_{\mathrm{cl}}$ which satisfies the potential energy shaping partial differential equations.

3. Total energy shaping: The intersection $S_{P} \cap S_{K}$ yields the set of $\lambda$ such that

(a) there exists a closed-loop metric which is a solution to the kinetic energy shaping problem and

(b) more importantly, potential energy shaping is possible, and as a result energy shaping is possible.

4. Determine the set of closed-loop potential functions $V_{\mathrm{cl}}$ with positive definite Hessian at the desired point. It would be interesting to have a geometric characterization of this.

\section{REFERENCES}

[1] R. Abraham, J. E. Marsden, and T. S. Ratiu, Manifolds, Tensor Analysis, and Applications, no. 75, Applied Mathematical Sciences, Springer-Verlag, second edition, 1988.

[2] J. A. Acosta, R. Ortega, A. Astolfi, and A. D. Mahindrakar, Interconnection and damping assignment passivity-based control of mechanical systems with underactation degree one, IEEE, Transactions on Automatic Control, 50 (2005), pp. 1936-1955.

[3] D. R. Auckly and L. V. Kapitanski, Mathematical problems in the control of underactuated systems, Nonlinear Dynamics and Renormalization Group, I. M. Signal and C. Sulem, eds., CRM Proc. Lecture Notes 27, AMS, Providence, RI, (2001), pp. 29-40.

[4] - On the $\lambda$-equations for matching control laws, SIAM Journal of Control and Optimization, 41 (2002), pp. 1372-1388.

[5] D. R. Auckly, L. V. Kapitanski, and W. White, Control of nonlinear underactuated systems, Communications on Pure and Applied Mathematics, 53 (2000), pp. 354-369.

[6] G. Blankenstein, R. Ortega, and A. J. van der Schaft, The matching conditions of controlled Lagrangians and IDA-passivity based control, International Journal of Control, 75 (2002), pp. 645-665.

[7] A. M. Bloch, D. E. Chang, N. E. Leonard, and J. E. Marsden, Controlled Lagrangians and the stabilization of mechanical systems. II. Potential shaping, IEEE Transactions on Automatic Control, 46 (2001), pp. 1556-1571.

[8] A. M. Bloch, N. E. Leonard, and J. E. Mardden, Controlled Lagrangians and the stabilization of mechanical systems. I. The first matching theorem, IEEE Transactions on Automatic Control, 45 (2000), pp. 2253-2270.

[9] R. Brockett, Control theory and analytical mechanics, The Ames Research Center (NASA) Conference on Geometric Control Theory (Moffett Field, Calif.), (1977), pp. 1-48.

[10] R. L. Bryant, S. S. Chern, R. B. Gardner, H. L. Goldschmidt, and P. A. Griffiths, Exterior Differential Systems, no. 18, Mathematical Sciences Research Institute Publications. New York-Heidelberg-Berlin: Springer-Verlag, 1991.

[11] F. Bullo And A. D. Lewis, Geometric Control of Mechanical Systems: Modeling, Analysis, 
and Design for Simple Mechanical Control Systems, no. 49 in Texts in Applied Mathematics, Springer-Verlag, 2004.

[12] A. Cabras, The Ricci identity for general connections, Proceeding of Conference on Differential Geometry and Applications, Brno, Czech Republic, (1995), pp. 121-126.

[13] D. E. Chang, Controlled Lagrangian and Hamiltonian systems, $\mathrm{PhD}$ thesis, California Institute of Technology, 2002.

[14] D. E. Chang, A. M. Bloch, N. E. Leonard, J. E. Marsden, and C. A. Woolsey, The equivalence of controlled Lagrangians and controlled Hamiltonian systems, ESAIM: Control, Optimization and Calculus of Variations, 8 (2002), pp. 393-422.

[15] M. DAlsmo And A. J. VAn DER Schaft, On representations and integrability of mathematical structures in energy conserving physical systems, SIAM Journal on Control and Optimization, 37 (1990), pp. 54-91.

[16] L. P. Eisenhart And O. Veblen, The Riemannian geometry and its generalization, Proceeding of the National Academy of Sciences of the United State of America, 8 (1922), pp. 19-22.

[17] W. Fulton, Young Tableaux : With Applications to Representation Theory and Geometry, Cambridge University Press, 1997.

[18] H. L. Goldschmidt, Existence theorems for analytic linear partial differential equations, Annals of Mathematics. Second Series, 86 (1967), pp. 246-270.

[19] — Integrability criteria for systems of nonlinear partial differential equations, Journal of Differential Geometry, 1 (1967), pp. 269-307.

[20] - Prolongation of linear partial differential equations. I, a conjecture of Elie Cartan, Annales Scientifiques de l'École Normale Supérieure. Quatrième Série, (1968), pp. 417444.

[21] V. Guillemin and M. Kuranishi, Some algebraic results concerning involutive subspaces, American Journal of Mathematics, 90 (1968), pp. 1307-1320.

[22] W. Guillemin and S. Sternberg, An algebraic model of transitive differential geometry, American Mathematical Society. Bulletin. New Series, 70 (1964), pp. 16-47.

[23] I. Kolár̆, P. W. Michor, And J. Slovák, Natural Operations in Differential Geometry, Springer-Verlag, Berlin, 1993.

[24] A. D. LEwis, Notes on energy shaping, 43rd IEEE Conference on Decision and Control, (2004), pp. 4818-4823.

[25] — Potential energy shaping after kinetic energy shaping, 45th IEEE Conference on Decision and Control, (2006), pp. 3339-3344.

[26] R. Ortega, M. W. Spong, F. Gomez-Estern, and G. Blankenstein, Stabilization of a class of underactuated mechanical systems via interconnection and damping assignment, IEEE Transactions on Automatic Control, 47 (2002), pp. 1218-1233.

[27] J. F. Pommaret, Systems of Partial Differential Equations and Lie Pseudogroups, Mathematics and its Applications, Gordon and Breach Science, 1978.

[28] - Partial Differential Equations and Group theory: New Perspectives for Applications, Mathematics and its Applications, Kluwer Academic Publishers, 1994.

[29] S. Prajna, A. J. van der Schaft, and G. Meinsma, An LMI approach to stabilization of linear port-controlled hamiltonian systems, Systems and Control Letters, 45 (2002), pp. 371-385.

[30] D. J. Saunders, Jet fields, connections and second order differential equations, Journal of Physics A: Mathematical and Theoretical, 20 (1987), pp. 3261-3270.

[31] - The Geometry of Jet Bundles, Cambridge University Press, 1989.

[32] I. M. Singer And S. Sternberg, The infinite group of Lie and Cartan, Journal d'Analyse Mathematique, 15 (1965), pp. 1-114.

[33] D. C. Spencer, Deformation of structures on manifolds defined by transitive, continuous pseudogroups. I. infinitesimal deformations of structure, Annals of Mathematics. Second Series, 
76 (1962), pp. 306-398.

[34] - Overdetermined systems of linear partial differential equations, American Mathematical Society. Bulletin. New Series, 75 (1967), pp. 159-193.

[35] M. Takegaki and S. Arimoto, A new feedback method for dynamic control of manipulators, American Society of Mechanical Engineers. Transactions of the ASME. Series G. Journal of Dynamical Systems and Measurement Control, 102 (1981), pp. 119-125.

[36] G. Thompson, Metrics compatible with a symmetric connection in dimension three, Journal of Geometry and Physics, 19 (1996), pp. 1-17.

[37] A. J. VAN DER SChAFt, Stabilization of Hamiltonian systems, Nonlinear Analysis, Theory, Methods and Applications, 10 (1986), pp. 1021-1035.

[38] G. Viola, R. Ortega, B. Banavar, J. A. Acosta, and A. Astolfi, The energy shaping control of mechanical systems: simplifying the matching equations via coordinate changes, IEEE Transaction on Automatic Control, 52 (2007), pp. 1093-1099.

[39] C. Woolsey, C. K. Reddy, A. Bloch, D. E. Chang, N. E. Leonard, and J. E. Marsden, Controlled Lagrangian systems with gyroscopic forcing and dissipation, European Journal of Control, 10 (2004).

[40] D. V. Zenkov, Matching and stabilization of linear mechanical systems, MTNS, 2002. 